SEUE INSTRUCTOR
ON
LUMBER SURVEYING.

\title{
QThice 50.0 .0$.
}

\section{S D \\ 555 \\ K56}




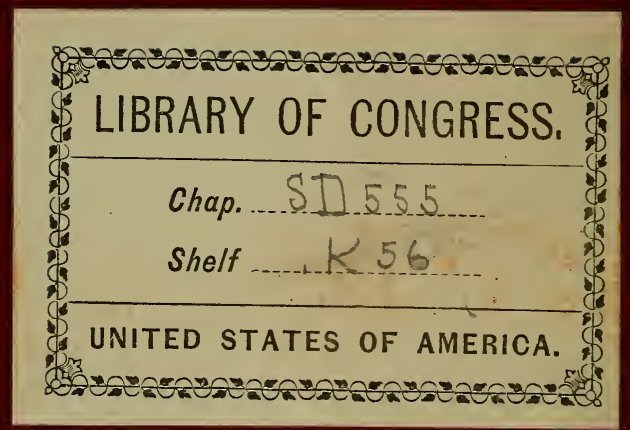








\section{SELF-INSTRUCTOR}

ON

\section{LUMBER SURVEYING,}

FOR THE USE OF

LUMBER MANUFACTURERS, SURVEYORS, AND TEACHERS.

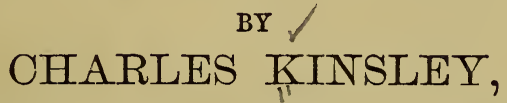

PRACTICAL SURVEYOR AND TEACHER OF SURVEYING.

ASSIGNOR,

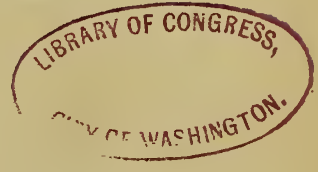

JAMES KINSLEY.

PUBLISHED BY THE AUTHOR.

Calais, Me., and St. Stephex, N. B.

1870. 


\section{$5 \pi 555$}

Entered according to Act of Congress, in the year 1870, By Charles Kinslex,

In the Office of the Librarian of Congress, at Washington.

RIVERSIDE, CAMBRIDGE:

ELECTROTYPED AND PRINTED BY

II. о. HOUGHTON AND COMPANY. 


\section{PREFACE.}

THIs work combines the theoretical and practical parts of surveying, in such a manner as to enable the energetic and uninitiated student who applies himself to the study of this useful and interesting science for a short time, to survey all kinds of lumber with accuracy and expertness. It contains tables for measuring boards, plank, deal, and timber by board measure, by which the Surveyor can dispense with the use of the Board Rule. It contains the rules generally adopted by Surveyors, and also a more concise rule than that in general use : for plank, deal, and timber, this rule alone is worth more than the price of the book to any Surveyor, as it requires less mental calculation than by the other rules, enabling him to survey faster and with less trouble than he could otherwise do. It contains tables for inch, inch and a quarter, and inch and a half boards for battens and joist. It also contains rules and tables for surveying logs by board and cubic measure, and rules for ton timber. It also contains tables showing the number of feet in length, of any dimension, which will make 1,000 feet board measure or 1,000 feet cubic measure; 
a new method of finding the solid contents of timber; a rule for finding what a round log will square, by having the circumference or diameter given, or in other words, to find the inscribed square; how to make out specifications, survey bills, etc.; rule for measuring tapering timber; table of quarter-girts for logs; rule for finding how much in length, of any dimension, which will make a solid foot, or any other desired quantity; table showing the weight of twenty-five kinds of wood, with a rule for finding the weight of the same from the contents; the English and American Government rules for finding the tonnage of vessels, and rules for gauging and ullaging casks. It also contains a correct and extensive interest table. 


\section{TABLE OF CONTENTS.}

Page

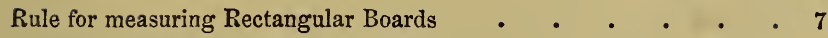

Exercise on the Rule . . . . . . . . . . 7

To find the Contents of a Triangular Board . . . . . 8

Exercise on the Rule . . . . . . . . . . 8

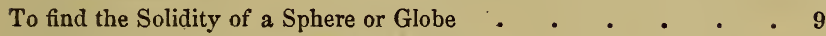

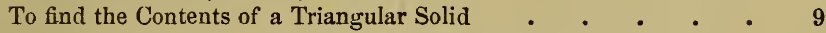

To find the Superficial Contents of a Globe . $\quad$. $\quad$. $\quad$. 9

To find the Contents of a Circular Board . $\quad$ - $\quad$. $\quad$. 9

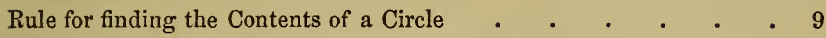

Rule for finding the Diameter or Circumference of a Circle $\quad$. $\quad 9$

Table for measuring Inch Boards, without a Board Rule, from Two

Inches to Thirty-six Inches wide . . . . . . . 10

Exercise on the Table . $\quad . \quad$. . . . . 10

Table for measuring Inch-and-a-Quarter Boards without a Board Rule 11

Exercise on the Table . . . . . . . . . . 11

Table for Inch-and-a-Half Boards without using the Board Rule . $\quad 12$

Exercise on the Rule . . . . . . . . . . 12

Table for Plank from Two to Thirty Inches wide . . . . . 13

Exercise on the Table . . . . . . . . . . 13

Table for Three-inch Deals from Three to Twenty-four Inches wide $\quad 14$

Table for Four-inch Deals from Four to Twelve Inches wide . $\quad 15$

Table for Five-inch Timber from Five to Twelve Inches wide . 16

Table for Six-inch Timber from Six to Twelve Inches wide . $\quad 16$

Table for Seven-inch Timber from Seven to Twelve Inches wide . $\quad$ 17

Table for Eight-inch Timber from Eight to Twelve Inches wide $\quad$. 17

Table for Nine-inch Timber from Nine to Twelve Inches wide . $\quad 17$

Table for Ten-inch Timber from Ten to Twelve Inches wide . $\quad$. 17

Table for Eleven-inch Timber from Eleven to Twelve Inches wide $\quad 17$

Table for Twelve-inch Timber from Twelve to Twenty Inches wide . 17

Plan of drawing a Plank Shingle, with Directions for dotting Plank,

Deal, \&c. . . . . . . . . . . 18, 19

Rule for Plank Specifications . • • . • • • 20

How to keep a Joist or Scantling Shingle . • • • • 22

New York Deals, Three-inch . • . . . . . 23

Specification Rule for Three-inch . . . . . . . . 24

Specification Rules for Four-inch _ . • • • • . 25,26

Five-inch Timber Shingle, with Rule for Specification . . 28-30 
Rule for Six-inch Specification . • . . . . . 31 Rule for Seven-incli Specification . • • • • • . 33

Rule for Eight-inch Specification _ . • • • • 35

Rule for Nine-inch Specification . • • • • • • 37

Rule for Ten-inch Specification . • • • • • • . 40

Rule for Eleven-inch Specification . • . . . . . . 41

Rule for Twelve-inch Specification . • • • • • • 42

Rule for finding the Contents of Battens or Two-and-a-Half-inch Stuff 44

Specification of Batten Shingle and Rule • • • • • 45

Random Shingle, Contents given in the Columns . . . . 45

Random Shingle, Running Lengths in the Columns • • . 46

Table showing the Number of Feet in Length, of all Dimensions, that will make 1,000 feet of Board Measure . • . . . 47

Table showing the Number of Feet in Length. of all Dimensions, from Five Inches by Five Inches to Twenty-two Inches by TwentyFour Inches that will make 1,000 Cubic feet, with Rules showing how both Tables are computed . . . . . . . 48

New Rules for finding the Contents in Cubic Feet of Timber, from Five

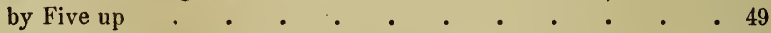

Second Metlıod of making out Specification, and Rule _ . . . 51,52 Specification of Philadelphia Deal, and Rule . . . . . 53,54 How to use the Board Riule, with Exercise . . . . . 54 Rule for measuring Logs, with Example . • • • • $\quad 55$

To find the Largest Square Piece of Timber that can be sawed from

a Round Log, by having the Circumference or Diameter given .

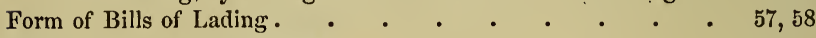

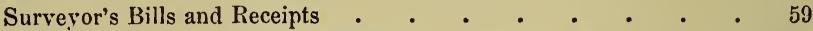

New Rules for finding the Superficial Contents of Plank, Deal, Joist,

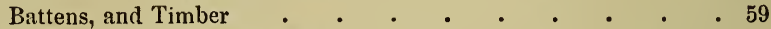

Given the Dimensions of the End of a Plank to find what Length of

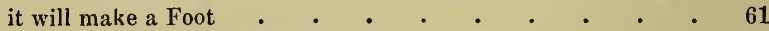

To find the Solid Contents of a Piece of Tapering Timber . . 61

When a Board or Plank is wider at one End than the other, to find what Length of it will make a Foot, or any Desired Quantity .

To find how much in Length will make a Solid Foot, or any other Desired Quantity, of Squared Timber of Equal Dimensions from End to End . . . . . . . . . . . . 62

Table for measuring Round Timber by the Quarter-girt Areas . $\quad$. 63

Table and Rule for finding the Weight of Timber from a Survey of its Contents . • • • • • • • • • • • 64

English Government Rule for finding the Tonnage of Vessels . $\quad 65$ United States Government rule for finding the Tonnage of Vessels . 66

Gauging of Casks • • • • • • • • • 67 . 67

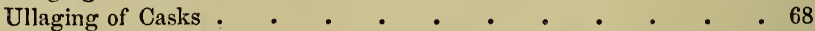

Questions for Exercise $. \quad . \quad . \quad . \quad . \quad$. 69

Log Rule for Round Timber . . . . . . . 72-76

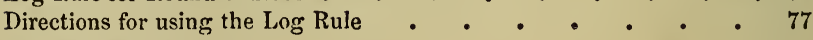

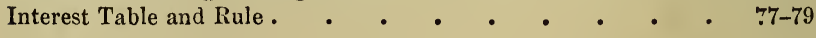




\section{SELF-INSTRUCTOR}

\section{ON \\ LUMBER SURVEYING.}

Rule for measuring Rectangular Boards.

Multiply the length in feet by the width in inches, and divide the product by 12 , to find the contents in superficial feet. Or multiply the length in inches by the width in inches, and divide by 144, the number of inches in a square foot, for the contents in superficial feet.

P. S. - A Rectangle is a plain figure bounded by four straight lines, which are equal and parallel, and whose angles are right angles, as $\mathrm{B}$.

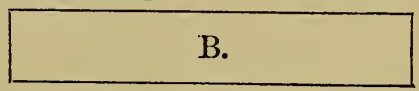

\section{QUESTIONS FOR EXERCISE.}

1. What are the contents in feet of a rectangular board 30 feet long and 20 inches wide? Ans. 50 feet.

2. How many feet in a board 26 feet 6 inches long, 12 inches in width?

Ans. $26 \frac{1}{2}$ feet.

3 . What will be the cost of a walnut board 32 feet long and 16 inches wide, at 8 cents per square foot. Ans. $\$ 3.41$.

4. What are the contents of a board 22 feet 8 inches long, and 1 foot 9 inches in width? Ans. 39 feet 8 inches.

When a Board is wider at one End than at the other.

Rule. - Add the width of both ends together, and take half the sum for a mean width, and multiply the width thus found by the length, for the contents ; or take the width in 
the middle of the board and multiply by the length, for the contents.

\section{EXAMPLE.}

1. What are the contents of a board 14 inches at one end and 20 inches at the other, and 24 feet in length.

Ans. 34 feet.

$14+20=34 \div 2=17$, mean width in inches, which multiplied by the length, 24 feet $=408 ; 408 \div 12=34$ feet $=$ contents.

2. What are the contents of a board 26 feet long, which measures 16 inches in the middle? Ans. 34 feet 8 inches.

26 feet $\times 16=416 ; 416 \div 12=34$ feet 8 inches $=$ contents.

\section{To find the Contents of a Triangular Board.}

Rule. - Multiply the length in feet by the width in inches, and take half the sum for the contents in inches, which being divided by 12 will give the contents in feet of board measure.

\section{EXAMPLE.}

1. What are the contents of the board A B C, whose base B C is 26 inches, and perpendicular height A D is 18 feet. Ans. 19 feet 6 inches.

$18 \times 26=468 \div \frac{1}{2}=234 \div 12$ $=19$ feet 6 inches.

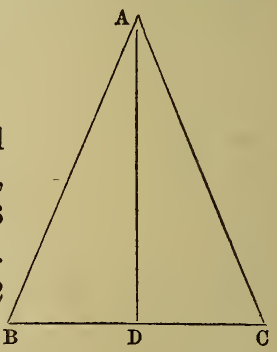

2. What are the contents of the triangular board A B C, whose base B C is 2 feet 6 inches, and perpendicular A, C, 24 feet. Ans. 30 feet.

24 feet $\times 2 \frac{1}{2}=60$ feet; 60 feet $\div \dot{2}$ $=30$ feet. Or -

2 feet 6 inches $=30$ inches; 30 inches $\times 24$ feet $=720$ inches $; 720 \div 2=360$ inches $=$ contents $; 360 \div 12=30$ feet $_{\mathbf{B}}$ $=$ contents in feet. 
The contents of a triangular solid can be found in the same manner by the foregoing rule, by multiplying the contents thus found by the thickness of the solid.

How many feet of boards in a triangular piece of timber, A B C, whose length A B is 24 feet, breadth B C 18 inches, and thickness C E 2 feet 6 inches?

24 feet $\times 18$ inches $=432 ; 432 \div 2$ $=216$ inches ; 216 inches $\div 12=18$ feet $=$ contents of superficial triangle A B $\mathrm{C}$, which being multiplied by the thickness $\mathrm{C} \mathrm{E}, 2$ feet 6 inches, will give the contents

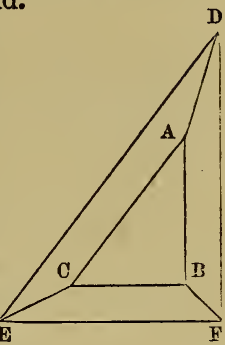
of the solid triangle A B C D E F, 18 feet $\times 2 \frac{1}{2}$ feet $=$ Ans. 45 cubic feet, or 540 board measure.

\section{For Measurement of a Globe.}

Rule. - To find the solidity of a globe, cube the diameter, and multiply the product by 5,236; and to find the surface of a globe, multiply the diameter by the circumference. To find the circumference by having the diameter given, say as 7 is to 22 , so is the diameter to the circumference, or as 22 is to 7 , so is the circumference to the diameter.

To find the Contents of a Circle.

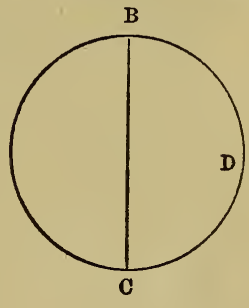

Rule 1. - Multiply half the circumference by half the diameter, for the contents.

Rule 2. - Square the diameter, and multiply it by $\mathbf{. 7 8 5 4}$ for the contents, or square the circumference, and multiply it by .07958 for the contents.

$P$. S. - The square of a number is found by multiplying the number by itself. 
Table for measuring Inch Boards without a Rule, from 2 Inches to 36 Inches wide.

\begin{tabular}{|c|c|c|c|}
\hline aes. & hes. & Inches. & ches. \\
\hline$=$ & $11 \times 1=$ & $20 \times$ & $29>$ \\
\hline & $=$ & $21 \times 1=1$ & $1=2$ \\
\hline 4 & $=1 \frac{1}{1}$ & $22 \times 1=1 \frac{5}{6}$ & $31 \times 1=27_{1}^{7}$ \\
\hline$=\frac{5}{12}$ & $14 \times 1$ & $23 \times 1=1 \frac{1}{1} \frac{1}{2}$ & $32 \times 1=2 \frac{2}{3}$ \\
\hline 1 & $15)$ & $24 \times 1=$ & $33 \times 1=2$ \\
\hline$=\frac{7}{12}$ & $16 \times 1=1 \frac{1}{3}$ & $25 \times 1=2 \frac{1}{2}$ & $1=2$ \\
\hline & $17 \times 1=1 \frac{5}{12}$ & $26 \times 1=2 \frac{1}{6}$ & $=2 \frac{1}{1} \frac{1}{2}$ \\
\hline $9 \times$ & $18 \times$ & $27 \times 1=2 \frac{1}{4}$ & $=3$ \\
\hline$\times 1$ & $19 \times 1=1 \frac{7}{12}$ & $28 \times 1=2 \frac{1}{3}$ & \\
\hline
\end{tabular}

In order to survey boards by the Table of Board Measure, the Surveyor must commit the table to memory, and by a little practice, he will become expert at surveying by this method.

\section{Questions for Exercise done by the Table of Board Measure.}

1. What are the contents of a board 24 feet long and 18 inches wide?

Ans. $24 \times 1 \frac{1}{2}=36$ feet.

2. How many feet in a board 32 feet long and 17 inches wide? Ans. $45 \frac{1}{3}$ feet.

By the table, 17 inches wide is $1 \frac{5}{12}$ the length, for the contents; therefore 32 feet $\times 1 \frac{5}{12}=45 \frac{1}{3}$ feet.

3. What are the contents of a board 21 feet 6 inches long and 6 inches wide?

Ans. 10 feet 9 inches.

By the table, 6 inches wide is half the length, for the contents ; therefore 21 feet 6 inches $\div 2=10$ feet 9 inches $=$ contents.

4. Required the contents of a board 36 feet long and 3 inches wide? Ans. $36 \div 4=9$ feet.

5. Find the contents of a board 24 feet 8 inches long and 14 inches wide?

Ans. 24 feet 8 inches $\times 1 \frac{1}{6}=28$ feet 9 inches $4^{\prime \prime}$. 
6. Required the contents of a board 27 feet long and 30 inches wide?

Ans. $67 \frac{1}{2}$ feet.

7. What is the value of a walnut board 23 feet 6 inches long, and 36 inches wide, @ $12 \frac{1}{2}$ cents per square foot?

Ans. $\$ 8.81 \frac{1}{2}$.

8. Required the contents of a board 16 feet long and 27 inches wide?

Ans. 36 feet.

9. How many feet in a board 38 feet long and 28 inches wide?

Ans. 88 feet 8 inches.

10. Required the contents of a board 16 feet long and 19 inches in width?

Ans. 25 feet 4 inches.

Table for Inch-and-a-Quarter Boards, from 2 Inches to. 36 Inches wide.

\begin{tabular}{|c|c|c|}
\hline $\begin{array}{l}\text { Inches. } \quad \text { Feet. } \\
2 \times 1 \frac{1}{4}=\frac{5}{24} \\
3 \times 1 \frac{1}{4}=\frac{5}{16} \\
4 \times 1 \frac{1}{4}=\frac{5}{12} \\
5 \times 1 \frac{1}{4}=\frac{25}{4} \\
6 \times 1 \frac{1}{4}=\frac{5}{8} \\
7 \times 1 \frac{1}{4}=\frac{3}{4} \frac{5}{8} \\
8 \times 1 \frac{1}{4}=\frac{5}{6} \\
9 \times 1 \frac{1}{4}=\frac{4}{4} \\
10 \times 1 \frac{1}{4}=1 \frac{1}{24} \\
11 \times 1 \frac{1}{4}=1 \frac{7}{4} \\
12 \times 1 \frac{1}{4}=1 \frac{1}{4} \\
13 \times 1 \frac{1}{4}=1 \frac{1}{4}\end{array}$ & 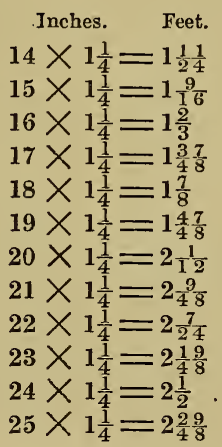 & 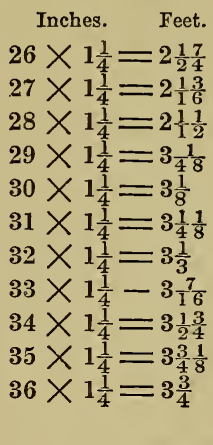 \\
\hline
\end{tabular}

Examples of 1 $1 \frac{1}{4}$-inch Board Measure done by the Table.

1. What are the contents of a board $1 \frac{1}{4}$ inches thick, 32 inches wide, and 30 feet long?

Ans. 100 feet.

By the table 32 inches is $3 \frac{1}{3}$ times the length; for the contents, therefore, 30 feet $\times 3 \frac{1}{3}=100$ feet.

2. What are the contents of a board $1 \frac{1}{4}$ inches by 18 inches, and 36 feet in length?

Ans. 67 feet 6 inches. 
3. Required the contents of a board $1 \frac{1}{4}$ inches by 24 inches, and 32 feet 8 inches in length?

Ans. 81 feet 8 inches.

4. How many feet in a $1 \frac{1}{4}$-inch board 16 inches wide and 24 feet long?

Ans. 40 feet.

5 . What will be the cost of a piece of mahogany $1 \frac{1}{4}$ inches by 12 inches, and 36 feet long, @ 6 cents per foot?

Ans. $\$ 2.70$.

Table for One-and-a-Half-inch Boards, from 2 to 24 Inches wide.

\begin{tabular}{|c|c|c|c|}
\hline $\begin{array}{l}\text { Inches. Feet. } \\
2 \times 1 \frac{1}{2}=\frac{1}{4} \\
3 \times 1 \frac{1}{2}=\frac{3}{8} \\
4 \times 1 \frac{1}{2}=\frac{1}{2} \\
5 \times 1 \frac{1}{2}=\frac{5}{8} \\
6 \times 1 \frac{1}{2}=\frac{3}{4} \\
7 \times 1 \frac{1}{2}=\frac{7}{8}\end{array}$ & $\begin{array}{l}\text { Inches. Feet. } \\
8 \times 1 \frac{1}{2}=1 \\
9 \times 1 \frac{1}{2}=1 \frac{1}{8} \\
10 \times 1 \frac{1}{2}=1 \frac{1}{4} \\
11 \times 1 \frac{1}{2}=1 \frac{3}{8} \\
12 \times 1 \frac{1}{2}=1 \frac{1}{2} \\
13 \times 1 \frac{1}{2}=1 \frac{5}{8}\end{array}$ & $\begin{array}{l}\text { Inches. Feet. } \\
14 \times 1 \frac{1}{2}=1 \frac{3}{4} \\
15 \times 1 \frac{1}{2}=1 \frac{7}{8} \\
16 \times 1 \frac{1}{2}=2 \\
17 \times 1 \frac{1}{2}=2 \frac{1}{8} \\
18 \times 1 \frac{1}{2}=2 \frac{1}{4} \\
19 \times 1 \frac{1}{2}=2 \frac{3}{8}\end{array}$ & $\begin{array}{l}\text { Inches. Feet. } \\
20 \times 1 \frac{1}{2}=2 \frac{1}{2} \\
21 \times 1 \frac{1}{2}=2 \frac{5}{8} \\
22 \times 1 \frac{1}{2}=2 \frac{3}{4} \\
23 \times 1 \frac{1}{2}=2 \frac{7}{8} \\
24 \times 1 \frac{1}{2}=3^{*}\end{array}$ \\
\hline
\end{tabular}

1. What are the contents of a $1 \frac{1}{2}$-inch board 32 feet long and 24 inches wide? Ans. 32 feet $\times 3$ feet $=96$ feet.

2. Required the contents of a $1 \frac{1}{2}$-inch board 18 feet long and 18 inches wide? Ans. $40 \frac{1}{2}$ feet.

3. Find the contents of a board $1 \frac{1}{2} \times 10$ inches and 28 feet 8 inches in length? Ans. 35 feet 10 inches,

By the table $1 \frac{1}{2} \times 10$ is $1 \frac{1}{4}$ the length, for the contents. 28 feet 8 inches $\times 1 \frac{1}{4}=35$ feet 10 inches.

4. What are the contents of a board 24 feet long, 20 inches wide, and $1 \frac{1}{2}$ inches thick? Ans. 60 feet.

5. Required the contents of a board 16 inches wide, $1 \frac{1}{2}$ inches thick, and 27 feet long. Ans. 54 feet.

6. What is the value of a board 17 inches wide, and $1 \frac{1}{2}$ inches thick, and 20 feet long, at 6 cents per foot?

Ans. \$2.55.

* Equal three times the length, for contents. 
Table for Two-inch or Plank, from 2 to 30 Inches wide.

\begin{tabular}{l|l|l|l}
\hline Inches. $\quad$ Feet. & Inches. Feet. & Inches. Feet. & \multicolumn{1}{l}{ Inches. Feet. } \\
$2 \times 2=\frac{1}{3}$ & $2 \times 10=1 \frac{2}{3}$ & $2 \times 17=2 \frac{5}{6}$ & $2 \times 24=4$ \\
$2 \times 3=\frac{1}{2}$ & $2 \times 11=1 \frac{5}{6}$ & $2 \times 18=3$ & $2 \times 25=4 \frac{1}{6}$ \\
$2 \times 4=\frac{2}{3}$ & $2 \times 12=2$ & $2 \times 19=3 \frac{1}{6}$ & $2 \times 26=4 \frac{1}{3}$ \\
$2 \times 5=\frac{5}{6}$ & $2 \times 13=2 \frac{1}{6}$ & $2 \times 20=3 \frac{1}{3}$ & $2 \times 27=4 \frac{1}{2}$ \\
$2 \times 6=1$ & $2 \times 14=2 \frac{1}{3}$ & $2 \times 21=3 \frac{1}{2}$ & $2 \times 28=4 \frac{2}{3}$ \\
$2 \times 7=1 \frac{1}{6}$ & $2 \times 15=2 \frac{1}{2}$ & $2 \times 22=3 \frac{2}{3}$ & $2 \times 29=4 \frac{5}{6}$ \\
$2 \times 8=1 \frac{1}{3}$ & $2 \times 16=2 \frac{2}{3}$ & $2 \times 23=3 \frac{5}{6}$ & $2 \times 30=5$ \\
$2 \times 9=1 \frac{1}{2}$ & & &
\end{tabular}

\section{EXERCISE.}

1. Required the contents of a plank 18 feet long and $\mathbf{1 5}$ inches in width?

Ans. 45 feet.

By the table 15 inches wide is $2 \frac{1}{2}$ times the length, for the contents in feet of board measure; therefore 18 feet $\times 2 \frac{1}{2}$ $=45$ feet.

2. Required the contents of a plank 36 feet long and 12 inches wide at one end, and 16 inches at the other end? Ans. 84 feet.

12 inches +16 inches $=28$ inches ; 28 inches $\div 2=$ mean width 14 inches. By the table 14 inches is $2 \frac{1}{3}$ times the length ; therefore 36 feet $\times 2 \frac{1}{3}=84$ feet.

3. What is the value of a plank 24 feet long and 27 inches wide @ $3 \frac{1}{2}$ cents per foot?

Ans. $\$ 3.92$.

4. Required the contents of a plank 18 feet long and 4 inches wide?

Ans. $\frac{18}{1} \times \frac{2}{3}=\frac{36}{3}=12$ feet.

5. What are the contents of 1,860 feet running lengths of 2 inches $\times 2$ inches?

Ans. 620 feet.

Solution. $-1,860 \div \frac{1}{3}=620$ feet.

6 . In 2,500 feet running lengths how many feet contents of 2 inches $\times 12$ inches?

2,500 feet $\times 2=5,000$ feet, or $5 \mathrm{M}$. Ans. 5,000 feet or $5 \mathrm{M}$. 
Table for Three-inch Deals, from 3 to 24 inches wide.

\begin{tabular}{l|l|l|l}
\hline Inches. Feet. & Inches. Feet. & Inches. Feet. & Inches. Feet. \\
$3 \times 3=\frac{3}{4}$ & $3 \times 9=2 \frac{1}{4}$ & $3 \times 15=3 \frac{3}{4}$ & $3 \times 20=5$ \\
$3 \times 4=1$ & $3 \times 10=2 \frac{1}{2}$ & $3 \times 16=4$ & $3 \times 21=5 \frac{1}{4}$ \\
$3 \times 5=1 \frac{1}{4}$ & $3 \times 11=2 \frac{3}{4}$ & $3 \times 17=4 \frac{1}{4}$ & $3 \times 22=5 \frac{1}{2}$ \\
$3 \times 6=1 \frac{1}{2}$ & $3 \times 12=3$ & $3 \times 18=4 \frac{1}{2}$ & $3 \times 23=5 \frac{3}{4}$ \\
$3 \times 7=1 \frac{3}{4}$ & $3 \times 13=3 \frac{1}{4}$ & $3 \times 19=4 \frac{3}{4}$ & $3 \times 24=6$ \\
$3 \times 8=2$ & $3 \times 14=3 \frac{1}{2}$ & &
\end{tabular}

EXERCISE.

1. What are the contents of a deal 3 inches thick, 6 inches wide, and 30 feet long? Ans. 4 ó feet.

By the table $3 \times 6$ is $1 \frac{1}{2}$ times the length, for the contents ; therefore 30 feet $\times 1 \frac{1}{2}=45=$ contents.

2. What are the contents of a deal 3 inches $\times 12$ inches and $33 \frac{1}{3}$ feet long?

Ans. 100 feet.

3. In 2,700 feet of running lengths of 3 inches $\times 20$ inches, how many feet?

Ans. 13,500 feet.

By the table $3 \times 20$ is 5 times the length, for the contents; $2,700 \times 5=13,500$ feet.

4. Required the number of feet running lengths of $3 \times 4$ that will be equal to 2,000 feet running lengths of 3 inches $\times 10$ inches?

Ans. 5,000 feet.

5. What number of feet of running lengths of $2 \times 3$ will be equivalent to 24,000 feet running lengths of $3 \times 12$ inches.

Ans. 144,000 feet.

Solution. - By the table $3 \times 12$ is 3 times the length, for the contents; therefore 24,000 feet $\times 3=72,000$ feet $=$ contents of $3 \times 12$ inches, and by the table $2 \times 3$ is $=$ to half the length, for the contents; therefore $2 \times 3$ is 2 times the contents for the running lengths, consequently 72,000 feet $\times 2=144,000$ feet running length. 
ON LUMBER SURVEYING.

Table for Four-inch Deals, from 4 to 12 Inches wide.

\begin{tabular}{c|c|c|c}
\hline Inches. Feet. & Inches. Feet. & Inches. Feet. & Inches. Feet. \\
$4 \times 4=1 \frac{1}{3}$ & $4 \times 7=2 \frac{1}{3}$ & $4 \times 9=3$ & $4 \times 11=3 \frac{2}{3}$ \\
$4 \times 5=1 \frac{2}{3}$ & $4 \times 8=2 \frac{2}{3}$ & $4 \times 10=3 \frac{1}{3}$ & $4 \times 12=4$ \\
$4 \times 6=2$ & & & \\
\hline
\end{tabular}

EXERCISE.

1. What are the contents of a deal $4 \times 4$ inches, and 20 feet long? Ans. $26 \frac{2}{3}$ feet.

2. What are the contents of a deal $4 \times 5$ and 24 feet long?

Ans. 40 feet.

3. Required the contents of a deal $4 \times 6$ and 26 feet long?

Ans. 52 feet.

4. Required the contents of a deal 4 inches $\times 12$ inches and 30 feet long?

Ans. 120 feet.

5. What is the value of a piece of oak 36 feet long, 4 inches thick, and 11 inches wide, @ $4 \frac{1}{2}$ cents per square foot?

6. In 2,800 feet of running lengths of 4 inches $\times 12$ inches, how many feet of superficial measurement are there?

Ans. 11,200 feet.

7. How many feet running lengths of 4 inches $\times 12$ inches deals are equal to 3,000 feet running lengths of $2 \times$ 6 ? . Ans. 750 feet.

8. What is the amount of lumber in the following cargo, and its value @ $\$ 15.00$ per M?

Surveyed from Bennett \& Co., of Boston, Mass., to Ship Aurora, Capt. Jones, -

2,758 pieces $2 \times 8$ and 16 feet long.

3,800 pieces $4 \times 12$ and 30 feet long.

2,600 pieces $4 \times 10$ and 16 feet long.

$250 \mathrm{M}$ of Mer. spruce laths @ $\$ 2.50$ per M.

Ans. 653,497 feet of lumber. $250 \mathrm{M}$ laths.

Value of lumber, $\$ 9,802.45 \frac{1}{2}$

Value of laths,

625.00

$\$ 10,427.45 \frac{1}{2}$ 
Table of Five-inch Timber, from 5 to 12 Inches wide.

\begin{tabular}{l|l}
\hline Inches. Feet. & \multicolumn{1}{c|}{ Inches. Feet. } \\
$5 \times 5=2 \frac{1}{12}$ & $5 \times 9=3 \frac{3}{4}$ \\
$5 \times 6=2 \frac{1}{2}$ & $5 \times 10=4 \frac{1}{6}$ \\
$5 \times 7=2 \frac{1}{12}$ & $5 \times 11=4 \frac{7}{12}$ \\
$5 \times 8=3 \frac{1}{3}$ & $5 \times 12=5$ \\
\hline
\end{tabular}

Table of Six-inch Timber, from 6 to 12 Inches wide.

\begin{tabular}{l|l}
\hline Inches. Feet. & Inches. Feet. \\
$6 \times 6=3$ & $6 \times 10=5$ \\
$6 \times 7=3 \frac{1}{2}$ & $6 \times 11=5 \frac{1}{2}$ \\
$6 \times 8=4$ & $6 \times 12=6$ \\
$6 \times 9=4 \frac{1}{2}$ & \\
\hline
\end{tabular}

\section{EXERCISE.}

1. What are the contents of a piece of timber 5 inches $X$ 5 inches and 24 feet long?

Ans. 50 feet.

By the table $5 \times 5$ is $2 \frac{1}{2}$ times the length, for the contents; therefore 24 feet $\times 2 \frac{1}{12}=\frac{24}{1} \times \frac{25}{12}=\frac{600}{12}=50$ feet in board measure.

2. Required the contents of a joist $5 \times 8$ and 30 feet long? 30 feet $\times 3 \frac{1}{3}=100$ feet.

Ans. 100 feet.

3. Find the contents of a beam 6 inches $\times 8$ inches and 36 feet in length?

36 feet $\times 4=144$ feet.

4. How many running feet of 6-inch $\times 8$-inch timber are equal to 3,500 feet running lengths of $5 \times 12$ inches ?

Ans. 4,375 feet.

By the table $5 \times 12$ is 5 times the length, for the contents, and $6 \times 8=4$ times the length ; therefore 3,500 feet $\times 5=17,500$ feet $=$ contents of $5 \times 12$; then $17,500 \div$ $4=4,375$ feet $=$ the number of feet in length of $6 \times$ $8=3,500$ feet of $5 \times 12$. 
5. What will a beam cost 48 feet long, 6 inches by 11 inches, @ 3 $3 \frac{1}{2}$ cents per foot? Ans. $\$ 9.24$.

$48 \times 5 \frac{1}{2}$ feet $=264$ feet $=$ contents $; 264 \times 3 \frac{1}{2}$ cents $=$ $\$ 9.24$.

Table of Timber from $7 \times 7$ to $12 \times 20$.

\begin{tabular}{|c|c|c|}
\hline Seven-inch Timber. & Eight-inch Timber. & Nine-inch Timber. \\
\hline $\begin{array}{l}\text { Inches. Feet. } \\
7 \times 7=4 \frac{1}{12} \\
7 \times 8=4 \frac{2}{3} \\
7 \times 9=5 \frac{1}{4} \\
7 \times 10=5 \frac{5}{6} \\
7 \times 11=6 \frac{5}{12} \\
7 \times 12=7\end{array}$ & $\begin{array}{l}\text { Inches. } \quad \text { Feet. } \\
8 \times 8=5 \frac{1}{3} \\
8 \times 9=6 \\
8 \times 10=6 \frac{2}{3} \\
8 \times 11=7 \frac{1}{3} \\
8 \times 12=8\end{array}$ & $\begin{array}{l}\text { Inches. } \quad \text { Feet. } \\
9 \times 9=6 \frac{3}{4} \\
9 \times 10=7 \frac{1}{2} \\
9 \times 11=8 \frac{1}{4} \\
9 \times 12=9\end{array}$ \\
\hline Ten-inch Timber. & Eleven-inch Timber. & Twelve-inch Timber. \\
\hline $\begin{array}{l}\text { Inches. Feet. } \\
10 \times 10=8 \frac{1}{3} \\
10 \times 11=9 \frac{1}{6} \\
10 \times 12=10\end{array}$ & $\begin{array}{l}\text { Inches. } \quad \text { Feet. } \\
11 \times 11=10 \frac{1}{12} \\
11 \times 12=11\end{array}$ & $\begin{array}{l}\text { Inches. } \quad \text { Feet. } \\
12 \times 12=12 \\
12 \times 14=14 \\
12 \times 16=16 \\
12 \times 18=18 \\
12 \times 20=20\end{array}$ \\
\hline
\end{tabular}

1. What are the contents of a piece of timber 12 by 12 inches and 30 feet long?

Ans. 360 feet.

2. What are the contents of a beam 7 inches by 9 inches and 30 feet long?

Ans. $157 \frac{1}{2}$ feet.

3 . Required the contents of a piece of timber $9 \times 10$ inches and 40 feet long?

Ans. 300 feet.

By the table $9 \times 10=7 \frac{1}{2}$ times the length ; 40 feet $\times 7 \frac{1}{2}$ $=300$ feet.

4. In 2,500 feet contents of $9 \times 10$, how many feet running lengths of $9 \times 10$, and of 11 by 12 ?

Ans. Of $11 \times 12,227 \frac{3}{11}$ feet. Of $9 \times 10,333 \frac{1}{3}$ feet.

5. What is the cost of 2,000 feet running lengths of 12 . 
inch by 20 -inch timber @ 3 cents per foot of board measure? Ans. $\$ 1,200.00$.

6. Required the contents of a piece of pine timber 8 inches by 12 inches and 24 feet long? Ans. 192 feet.

7. What is the difference in feet of board measure between 2,000 feet running lengths of $9 \times 12$ and 2,000 feet running lengths of $12 \times 12$ ?

Ans. $12 \times 12$ is 6,000 feet more.

By the table $12 \times 12=12$ times the length, and $9 \times$ $12=9$ times ; therefore $12-9=3$ feet difference ; 2,000 $\times 3=6,000$ feet difference.

Example showing the Manner of Drawing or Ruling a Shingle for Plank or 2-inch, also the Mode of Dotting.

Rule. - Take a shingle and rule it, as shingle No. 1 is ruled, the dimensions along the top column, and the lengths down the side column; then take a pencil and make a dot, thus (.), for every plank, or deal, or piece of timber, as the case may be. Suppose I want to dot a $2 \times 6,22$ feet long, 3 times, I run along the top column of dimensions till I come to $2 \times 6$; I then go down said line till I come opposite 22 in the column of lengths, I then make three dots, thus (...). Then when I have finished dotting, I count all the dots, and place the figures as in the above shingle ; those figures I afterwards transfer to my specification, in order to find the contents of the whole quantity of pieces I have dotted.

P. S. - You can, if required, rule your shingle so as to include any length or dimension, and most shingles are drawn as shingle No 1 is. 
ON LUMBER SURVEYING.

Plank. Shingle, No. 1.

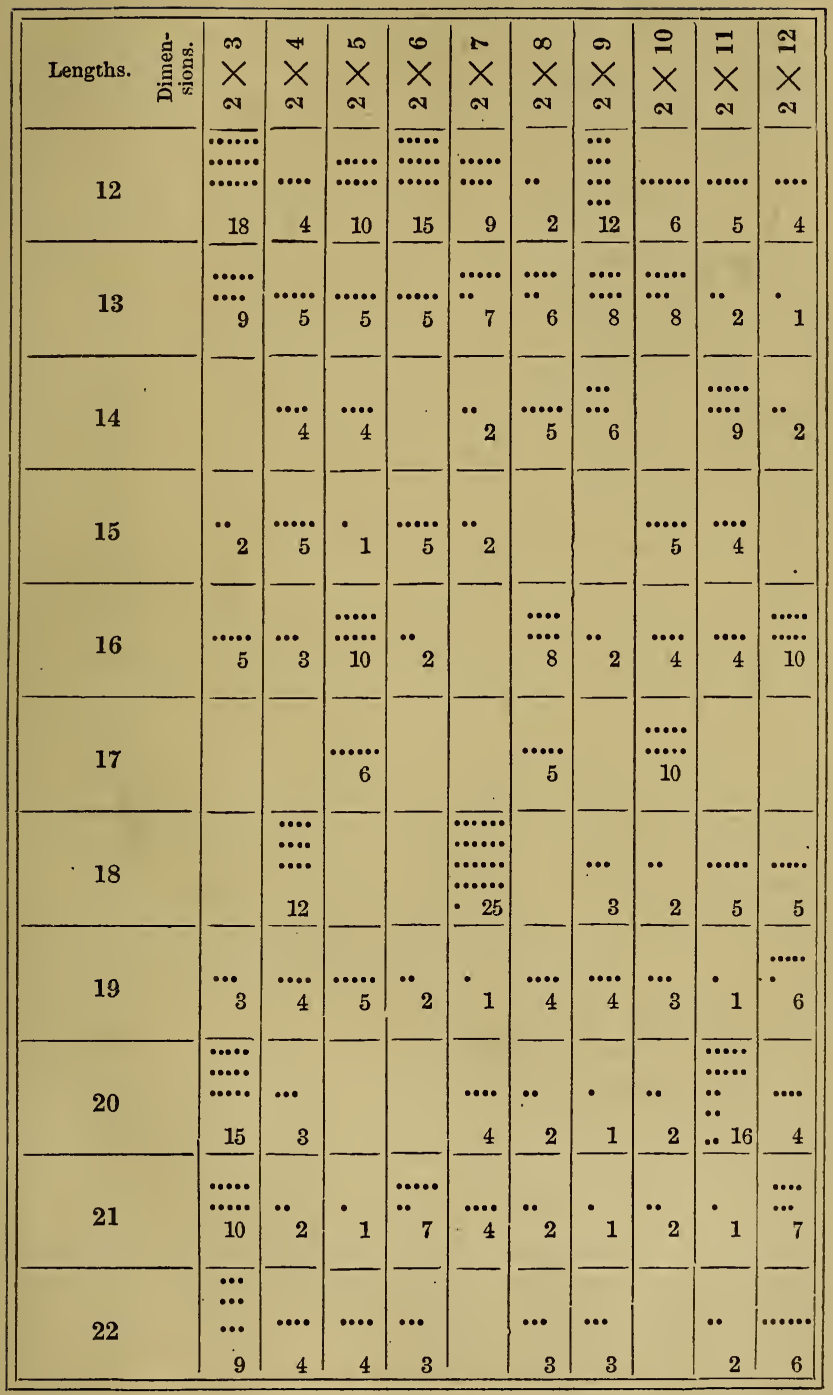


Example of Specification of the Plank Shingle No. 1, showing the manner of finding the Contents.

Rule. - One sixth of the length of 2-inch stuff multiplied by the width will give the contents in feet of board measure or superficial feet.

Specification of Plank Shingle No. 1.

\begin{tabular}{|c|c|c|c|c|c|c|c|c|c|c|c|}
\hline 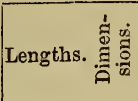 & $\begin{array}{l}\infty \\
X \\
\infty\end{array}$ & $\begin{array}{l}+4 \\
X \\
\text { N }\end{array}$ & $\begin{array}{l}0 \\
X \\
0\end{array}$ & $\begin{array}{l}0 \\
X \\
N\end{array}$ & $\begin{array}{l}\infty \\
x \\
0\end{array}$ & $\begin{array}{l}\infty \\
X \\
N\end{array}$ & $\begin{array}{l}0 \\
X \\
\text { ad }\end{array}$ & $\begin{array}{l}0 \\
-1 \\
X \\
\text { N }\end{array}$ & $\left|\begin{array}{l}F \\
X \\
\text { N }\end{array}\right|$ & $\begin{array}{l}0 \\
-1 \\
X \\
\text { N }\end{array}$ & Contents. \\
\hline 12 & 18 & 4 & 10 & 15 & 9 & 2 & 12 & 6 & 5 & 4 & 1,120 \\
\hline 13 & 9 & 5 & 5 & 5 & 7 & 6 & 8 & 8 & 2 & 1 & 834 \\
\hline 14 & & 4 & 4 & & 2 & 5 & 6 & & 9 & 2 & 623 \\
\hline 15 & 2 & 5 & 1 & 5 & 2 & & & 5 & 4 & & 422 \\
\hline 16 & 5 & 3 & $\overline{10}$ & -2 & - & 8 & $2^{\prime}$ & 4 & -4 & $\overline{10}$ & 1,000 \\
\hline 17 & & & 6 & & & 5 & & 10 & & & 482 \\
\hline 18 & & 12 & & & 25 & & 3 & 2 & 5 & 5 & 1,155 \\
\hline 19 & 3 & 4 & 5 & 2 & 1 & 4 & 4 & 3 & 1 & 6 & 792 \\
\hline 20 & 15 & 3 & & & 4 & 2 & 1 & 2 & 16 & 4 & 1,180 \\
\hline 21 & 10 & 2 & 1 & 7 & 4 & 2 & 1 & 2 & 1 & 7 & 885 \\
\hline 22 & 9 & 4 & 4 & 3 & & 3 & 3 & & 2 & 6 & 828 \\
\hline & & & & & & & & & & & Total, 9,321 feet. \\
\hline
\end{tabular}

Rule for calculating a 2-Inch or Plank Specification.

Multiply the number of pieces or dots in each square of the table by the width of said pieces, and the product by $\frac{1}{6}$ of the length for the contents. 
To find the Contents of Specification Shingle, No. 1.

Multiply the number of pieces in each square of the table, opposite the first length, 12 feet, by the widths of the different numbers of said pieces, and then by $\frac{1}{6}$ of the length for the contents; thus, for the first column running parallel to the top of the shingle,

$$
\begin{array}{lrrrrrrrrrr}
\text { Breadth. } & 3 & 4 & 5 & 6 & 7 & 8 & 9 & 10 & 11 & 12 \\
\text { No. Pieces. } & 18 & 4 & 10 & 15 & 9 & 2 & 12 & 6 & 5 & 4 \\
& \frac{54}{16} & \frac{50}{50} & \frac{7}{63} & \frac{7}{16} & \frac{108}{60} & \frac{-}{55} & \frac{18}{48}
\end{array}
$$

Then add all the products, $54+16+50+90+63+$ $16+108+60+55+48=560$. Then 12 , the length, $\div 6=2$ feet, $560 \times 2=1,120=$ contents of the first column. Thus proceed until the contents of all the columns are found, then add the whole together for the total contents of the shingle.

P. S. - In this treatise, when there is a fraction of half a foot over, it is called a foot; when less than half a foot, nothing.

\section{For Joist or Scantling.}

Take the running lengths of the different dimensions and mark down every 100 feet, then add up your shingle, and multiply the different sums by the multiplier of each dimension, as found in the tables for the contents of each. Hemlock joist is generally. computed by this plan. 
Joist Shingle.

\begin{tabular}{|r|c|c|c|c|c|c|}
\hline $2 \times 3$ & $2 \frac{1}{2} \times 3$ & $2 \times 4$ & $2 \frac{1}{2} \times 4$ & $2 \frac{1}{4} \times 3$ & $2 \frac{1}{4} \times 4$ & $3 \times 4$ \\
\hline 100 & 100 & 10 & 100 & 100 & 100 & 250 \\
100 & 100 & 90 & 100 & 100 & 100 & 250 \\
100 & 100 & 100 & 100 & 100 & 100 & 100 \\
50 & 100 & 100 & 100 & 100 & 100 & 100 \\
200 & 100 & 100 & 100 & 100 & 100 & 100 \\
100 & 150 & 100 & 100 & 100 & & 100 \\
100 & 100 & 100 & 100 & 100 & & \\
25 & 100 & 100 & 100 & 100 & & \\
150 & 100 & 100 & 100 & & & \\
200 & 50 & 100 & 100 & & & \\
100 & & 100 & & & & \\
\hline 613 & 625 & 667 & 833 & 450 & 375 & $900^{*}$ \\
\hline
\end{tabular}

* The numbers at the foot of the columns are feet of board measure.

3 inches by 4 inches by the table is once the length, therefore there are 900 feet of $3 \times 4$ contents. There are in the joist shingle 500 feet running length of $2 \frac{1}{4} \times 4$, and $2 \frac{1}{4} \times 4$ is $=\frac{3}{4}$ times the length; therefore, $500 \div \frac{3}{4}=$ to 375 feet $=$ contents of $2 \frac{1}{4} \times 4$. There are 800 feet running lengths of $2 \frac{1}{4} \times 3$, and $2 \frac{1}{4} \times 3$ is $\frac{9}{16}$ times the length; therefore, $800 \div \frac{9}{16}=450=$ contents. There are 1,000 feet of $2 \frac{1}{2} \times 4$; therefore, as $2 \frac{1}{2} \times 4$ is $\frac{5}{6}$ of the length, the contents will be equal to $1,000 \div \frac{5}{6}=833$ feet. Of 2 inches $\times 4$ inches, 1,000 feet, which divided by $\frac{2}{3}$, will be the contents $=667$ feet. Of $2 \frac{1}{2} \times 3$ there are 1,000 feet, and $2 \frac{1}{2} \times 3$ is $=\frac{5}{8}$ times the length ; therefore, $1,000 \div \frac{5}{8}$ $=625$ feet. Of $2 \times 3$ there are 1,225 feet running lengths, and $2 \times 3$ is $\frac{1}{2}$ the length; therefore, $1,225 \div \frac{1}{2}=612 \frac{1}{2}$ feet. 
New York Deal Shingle, 3-Inch, No. 2.

\begin{tabular}{|c|c|c|c|c|c|c|c|}
\hline Lengths. $\frac{\dot{d}}{\dot{0}} \dot{a}$ & $\underbrace{\infty}_{\infty}$ & $x_{0}^{x}$ & $\underbrace{\infty}_{\infty}$ & $\underbrace{\infty}_{\infty}$ & $\underset{\infty}{\stackrel{9}{e}}$ & $\underset{n}{\mp}$ & $\underset{\infty}{x}$ \\
\hline 14 & $\ldots \ldots \ldots$ & $\begin{array}{r}\cdots \cdots \cdots \\
14 . . . \\
\end{array}$ & $\begin{array}{r}\cdots \cdots \cdot \ldots . . . . \\
20 \\
\end{array}$ & $\begin{array}{r}16 . . . . \\
16 \\
\end{array}$ & $\begin{array}{r}18 \\
\end{array}$ & $\begin{array}{r}\cdots \cdots . . . \\
20 \\
\end{array}$ & $\cdots \cdots$ \\
\hline 15 & 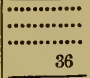 & $\begin{array}{r}0 . \ldots \ldots \ldots . . . \\
20 \\
\end{array}$ & $\begin{array}{r}\ldots \ldots \ldots \ldots \\
30 \\
\end{array}$ & 11 & $\begin{array}{r}\cdots \cdot . \\
5 \\
\end{array}$ & $\begin{array}{l}\cdots \\
3 \\
\end{array}$ & .... 4 \\
\hline 16 & $\begin{array}{c}\cdots \ldots . . \\
12\end{array}$ & & $\begin{array}{r}\cdots \cdots \cdot . \\
6 \\
\end{array}$ & $\begin{array}{r}r \ldots . . \\
8 \\
\end{array}$ & $\begin{array}{r}\ldots \ldots \ldots \\
10 \\
\end{array}$ & $\begin{array}{r}\cdot \\
1 \\
\end{array}$ & 7 \\
\hline 17 & & $\begin{array}{r}\cdots \cdots \\
9 \\
\end{array}$ & $\begin{array}{r}\ldots \ldots \ldots \ldots . . . \\
24\end{array}$ & $\begin{array}{r}\cdots \cdots \cdot . \\
15\end{array}$ & $\begin{array}{r}\cdots \cdots \cdot . . . \\
15 \\
\end{array}$ & $\begin{array}{r}\cdots . . . . . . \\
16 \\
\end{array}$ & $\begin{array}{c}\cdots \ldots \ldots \ldots \\
27 \ldots \ldots \\
27\end{array}$ \\
\hline 18 & $\begin{array}{l}\ldots \ldots \ldots \ldots \\
\cdots \ldots \ldots \ldots \\
\cdots \quad \ldots \ldots \\
\cdots \quad 26\end{array}$ & $\begin{array}{r}0 . \cdots \\
8\end{array}$ & $\begin{array}{r}\cdots \cdots \\
5 \\
\end{array}$ & $\begin{array}{r}\cdots . . . . . \\
9 \\
9\end{array}$ & 8 & $\begin{array}{r}\cdots \cdot \cdots \\
7 \\
\end{array}$ & $\begin{array}{r}\ldots \ldots \ldots . . \\
10 \\
\end{array}$ \\
\hline 19 & $\begin{array}{r}\ldots \ldots . . . \\
\ldots \ldots . . . \\
21\end{array}$ & …... & $\begin{array}{l}\cdots \\
3 \\
\end{array}$ & $\begin{array}{ll}* \\
\end{array}$ & $\begin{array}{ll}\cdot \\
\end{array}$ & 7 & $\begin{array}{l}\cdots . . \\
\quad 4 \\
\end{array}$ \\
\hline 20 & 12 & 12 & 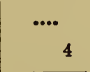 & $\begin{array}{ll}\cdots & \\
& 3\end{array}$ & ${ }_{4}$ & 4 & 10 \\
\hline 21 & & 16 & $\begin{array}{r}\ldots \ldots \ldots \ldots \\
27 \\
\end{array}$ & 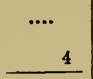 & $\begin{array}{l}\cdots \\
\end{array}$ & .... & .... \\
\hline 22 & 8 & ${ }^{\prime \cdots .} 4$ & ${ }_{3}$ & ${ }_{5}$ & $\cdots_{4}$ & 3 & …. \\
\hline 23 & $\begin{array}{c}0 \ldots \ldots \ldots \\
\ldots \ldots \ldots \ldots \\
27\end{array}$ & & $\begin{array}{r}20 . \cdots \cdot . \\
20 . \\
\end{array}$ & 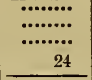 & $\begin{array}{l}\cdots \\
3 \\
\end{array}$ & 7 & $\begin{array}{r}20 \\
\end{array}$ \\
\hline 24 & & 15 & 4 & 4 & 8 & 4 & .... \\
\hline 25 & 6 & 10 & 4 & ${ }_{4}$ & ${ }_{3}$ & 9 & 8 \\
\hline 26 & ..... & 10 & 10 & $\begin{array}{r}\cdots . . . . . \\
9 \\
\end{array}$ & $\begin{array}{r}\cdots \cdots . . . . \\
20 \\
\end{array}$ & $\begin{array}{l}\cdots \cdot \\
\end{array}$ & $\begin{array}{r}\cdots \cdot .6 \\
6 \\
\end{array}$ \\
\hline 27 & 10 & 9 & ${ }_{4}$ & $\cdots$ & $\cdots_{4}$ & $\cdots$ & ${ }_{4}$ \\
\hline 28 & & $\begin{array}{c}\ldots . . . . . \\
18 \\
\end{array}$ & .... & 5 & .... & 4 & .... \\
\hline 29 & $\begin{array}{r}30 \\
0\end{array}$ & 11 & 9 & $\begin{array}{r}\cdots \\
12\end{array}$ & 5 & & 10 \\
\hline 30 & $\begin{array}{r}0 . \ldots \ldots \\
\quad \ldots \ldots \ldots \\
24\end{array}$ & $\begin{array}{r}\ldots . . . . . . . \\
20 \\
\end{array}$ & $\begin{array}{r}\cdots \cdot \ldots \\
8 \\
\end{array}$ & $\begin{array}{r}\cdots \cdots \\
5\end{array}$ & $\begin{array}{r}\cdots \cdot . \\
\quad 4 \\
\end{array}$ & 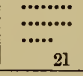 & $\begin{array}{r}\quad \ldots . . . . \\
9 \\
\end{array}$ \\
\hline
\end{tabular}


Specification of New York Deal Shingle, No. 2.

\begin{tabular}{|c|c|c|c|c|c|c|c|c|}
\hline 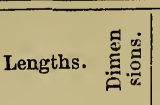 & ${ }_{\infty}^{0} \times$ & $\underset{\infty}{x}$ & $\underset{\infty}{\infty}$ & $\begin{array}{l}\infty \\
X \\
\infty\end{array}$ & $\begin{array}{l}\stackrel{9}{X} \\
\times \\
\infty\end{array}$ & $\underset{n}{\mp}$ & $\begin{array}{l}\stackrel{N}{*} \\
X \\
\text { X }\end{array}$ & Contents. \\
\hline 14 & 24 & 14 & 20 & 16 & 18 & 20 & 30 & 4,571 \\
\hline 15 & 36 & 20 & 30 & 11 & 5 & 3 & 4 & 3,098 \\
\hline 16 & 12 & & 6 & 8 & 10 & 1 & 7 & 1,548 \\
\hline 17 & & 9 & 24 & 15 & 15 & 16 & 27 & 4,420 \\
\hline 18 & 26 & 8 & 5 & 9 & 8 & 7 & 10 & 2,744 \\
\hline 19 & 21 & 12 & 3 & 2 & 1 & 7 & 4 & 1,838 \\
\hline 20 & 12 & 12 & 4 & 3 & 4 & 4 & 10 & 2,095 \\
\hline 21 & & 16 & 27 & 4 & 4 & 4 & 5 & 2,667 \\
\hline 22 & 8 & 4 & 3 & 5 & 4 & 3 & 12 & 1,991 \\
\hline 23 & 27 & 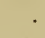 & 20 & 24 & 3 & 7 & 20 & 5,089 \\
\hline 24 & & 15 & 4 & 4 & 8 & 4 & 4 & 2,070 \\
\hline 25 & 6 & 10 & 4 & 4 & 3 & 9 & 8 & 2,494 \\
\hline 26 & 5 & 10 & 10 & 9 & 20 & 4 & 6 & 3,750 \\
\hline 27 & 10 & 9 & 4 & 5 & 4 & 5 & 4 & 2,315 \\
\hline 28 & & 18 & 4 & 5 & 4 & 4 & 5 & 2,429 \\
\hline 29 & 30 & 11 & 9 & 12 & 5 & & 10 & 4,401 \\
\hline 30 & 24 & 20 & 8 & 5 & 4 & 21 & 9 & 5,790 \\
\hline
\end{tabular}

Rule for finding the Contents of 3-Inch Deals.

Multiply $\frac{1}{4}$ of the length of the deals by the breadth of them, for the contents.

This shingle is done the same way as the plank shingle No. 1, excepting that $\frac{1}{4}$ of the lengths are taken instead of $\frac{1}{6}$ of them. 
ON LUMBER SURVEYING.

New York Deal Shingle, 4-Inch, No. 3.

\begin{tabular}{|c|c|c|c|c|c|c|c|}
\hline Lengths. & $\underset{x}{x}$ & $x$ & $\underset{+}{\infty}$ & $\ddot{x}$ & $\begin{array}{l}\frac{9}{x} \\
x\end{array}$ & $\begin{array}{l}\bar{z} \\
\bar{x}\end{array}$ & $\begin{array}{l}\tilde{N} \\
\bar{x}\end{array}$ \\
\hline 14 & 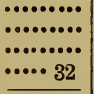 & $\begin{array}{r}0 . . . \\
\quad 10 \\
\end{array}$ & 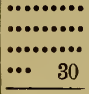 & $\begin{array}{r}\cdots \cdots . . \\
\cdots \cdots \\
12 \\
\end{array}$ & $\begin{array}{r}\cdots \cdots . . . \\
\cdots . . . \\
\quad 14 \\
\end{array}$ & $\begin{array}{r}\cdots \cdots . . . . . \\
\cdots . . . \\
17\end{array}$ & $\begin{array}{r}\cdots \cdots \\
-\quad 5 \\
\end{array}$ \\
\hline 15 & $\begin{array}{r}\cdots \cdots . . . . . . \\
\cdots \cdots . . . \\
24 \\
24 \\
\end{array}$ & $\begin{array}{r}\because \cdots \cdots \cdot . \cdot \\
\quad 10 \\
\end{array}$ & $\begin{array}{rr}\cdots & \\
& 11 . . . . \\
\end{array}$ & & $\begin{array}{r}\cdots \cdots \cdot . \cdots \\
\quad 10 \\
\end{array}$ & $\begin{array}{r}\cdots \cdots . . . . \\
16 \\
\end{array}$ & $\begin{array}{r}\cdots . . . . . \\
\cdots \cdots . . . \\
21 \\
\end{array}$ \\
\hline 16 & $\cdots_{4}$ & $i$ & $\cdots_{4}$ & $\cdots_{5}$ & $\cdots_{5}$ & $\cdots_{4}$ & ${ }_{13}$ \\
\hline 17 & $\begin{array}{r}\cdots \cdots \\
9\end{array}$ & & $\cdots$ & $\because . . . . . .$. & …............. & $\cdots . . . .$. & 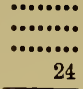 \\
\hline 18 & 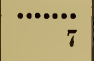 & $\begin{array}{ll}\cdot & 2\end{array}$ & - 1 & $\cdots_{4}$ & $\begin{array}{ll}\cdots & 3\end{array}$ & $\cdots_{5}$ & $\cdots_{4}$ \\
\hline 19 & $\cdots \cdots$ & $\cdots_{4}$ & $\cdots_{4}$ & $\cdots_{4}$ & $\because \cdots{ }_{10}$ & & $\cdots{ }_{5}$ \\
\hline 20 & $\ldots$ & $\cdots ._{6}$ & & $\begin{array}{ll}\cdots & 3\end{array}$ & $\begin{array}{ll}{ }^{*} & 2\end{array}$ & $\because \quad 10$ & …... \\
\hline 21 & 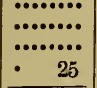 & & 8 & $\begin{array}{l}\cdots \\
\quad\end{array}$ & $\begin{array}{r}\cdots \cdot . \cdot \\
\quad 6 \\
\end{array}$ & $\begin{array}{l}\cdots \cdot . \cdot \\
\quad 5 \\
\end{array}$ & $\begin{array}{ll}\cdots & \\
& 4 \\
\end{array}$ \\
\hline 22 & & $\cdots_{6}$ & …... & $\cdots$ & ......... & 12 & ${ }_{11}$ \\
\hline 23 & $\begin{array}{ll}\cdots \\
\end{array}$ & $\begin{array}{ll}\cdots & \\
& 3\end{array}$ & $\begin{array}{ll}\bullet & 2\end{array}$ & $\begin{array}{ll}\cdots & 3\end{array}$ & $\cdot$ & $\begin{array}{ll}\ddot{ } \quad 2\end{array}$ & 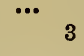 \\
\hline 24 & $\cdots{ }_{6}$ & $\cdots_{4}$ & $\cdots_{5}$ & $\begin{array}{ll}\cdots & 3\end{array}$ & $\begin{array}{lll}\cdots^{\cdots} & 3\end{array}$ & $\because \cdots \quad \ldots$ & $\cdots{ }_{6}$ \\
\hline
\end{tabular}

Rule for finding the Contents of 4-Inch Deals.

Multiply the length divided by 3 by the breadth for the contents in feet of board measure. 
What are the contents of 32 pieces 14 feet long and $4 \times 6$ ? $32 \times 14=448$ feet of running length, then $14 \div 3=4 \frac{2}{3}$ $=\frac{1}{3}$ of length of each piece. And $4 \times 6$ inches by the table is $=2$ times the length, for the contents, therefore $448 \times 2=896$ feet $=$ contents. By taking $\frac{1}{3}$ of the length, it is done thus, 32 pieces $\times 6$, their breadth $=$ $192 \times 4 \frac{2}{3}=896$ feet, contents. Or multiply the number of piecess by the length of one, and the product by $\frac{1}{3}$ of the width of the deals for the contents of 4 -inch.

Specification of New York Deal Shingle, 4-Inch, No. 3.

\begin{tabular}{|c|c|c|c|c|c|c|c|c|}
\hline 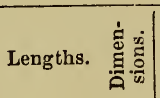 & $\begin{array}{l}\infty \\
X \\
\forall\end{array}$ & $\underset{\nabla}{x}$ & $\begin{array}{l}\infty \\
X \\
-\end{array}$ & $\begin{array}{l}a \\
X \\
H\end{array}$ & $\begin{array}{l}0 \\
x \\
x\end{array}$ & $\begin{array}{l}\bar{Z} \\
X \\
\not\end{array}$ & $\underset{\sim}{X}$ & Contents. \\
\hline 14 & 32 & 10 & 30 & 12 & 14 & 17 & 5 & 4,653 \\
\hline 15 & 24 & 10 & 11 & & 10 & 16 & 21 & 4,150 \\
\hline 16 & 4 & 7 & 4 & 5 & 5 & 4 & 13 & 2,133 \\
\hline 17 & 9 & & 7 & 11 & 18 & 14 & 24 & 4,709 \\
\hline 18 & 7 & 2 & 1 & 4 & 3 & 5 & 4 & 1,398 \\
\hline 19 & 7 & 4 & 4 & 4 & 10 & & 5 & 1,887 \\
\hline 20 & 8 & 6 & & 3 & 2 & 10 & 12 & 2,607 \\
\hline 21 & 25 & & 8 & 4 & 6 & 5 & 4 & 2,891 \\
\hline 22 & & 6 & 7 & 7 & 9 & 12 & 11 & 3,777 \\
\hline 23 & 8 & 3 & 2 & 3 & 1 & 2 & 3 & 1,380 \\
\hline \multirow[t]{2}{*}{24} & 6 & 4 & 5 & 3 & 3 & 11 & 6 & 2,832 \\
\hline & & & & & & & & Total, 32,417 feet. \\
\hline
\end{tabular}


Solution of Specification No. 3.

\begin{tabular}{|c|c|c|c|c|c|}
\hline No. & Products. & No. & Products. & No. & Products. \\
\hline $32 \times 6=$ & 192 & $9 \times 6=$ & 54 & $8 \times 6=$ & 48 \\
\hline $10 \times 7=$ & 70 & $7=$ & 56 & $6 \times 7=$ & 42 \\
\hline $5 \times 12=$ & 60 & $11 \times 9=$ & 99 & $3 \times 9=$ & 27 \\
\hline $30 \times 8=$ & 240 & $18 \times 10=$ & 180 & $2 \times 10=$ & 20 \\
\hline $12 \times 9=$ & 108 & $14 \times 11=$ & 154 & $10 \times 11=$ & 110 \\
\hline $14 \times 10=$ & 140 & $24 \times 12=$ & 288 & $12 \times 12=$ & 144 \\
\hline $17 \times 11=$ & 187 & & & & \\
\hline & 997 & $17 \div 3=$ & $5 \frac{2}{3}$ & $20 \div 3=$ & $6 \frac{2}{3}$ \\
\hline & & Contents, & 4,709 & Contents, & 2,607 \\
\hline Contents, & 4,653 & & & & \\
\hline $24 \times 6=$ & 144 & $\begin{array}{l}7 \times 6= \\
2 \times 7=\end{array}$ & $\begin{array}{l}42 \\
14\end{array}$ & $\begin{array}{r}25 \times 6= \\
8 \times 8=\end{array}$ & $\begin{array}{r}150 \\
64\end{array}$ \\
\hline $10 \times 7=$ & 70 & $1 \times 8=$ & 8 & $4 \times 9=$ & 36 \\
\hline $11 \times 8=$ & 88 & $4 \times 9=$ & 36 & $6 \times 10=$ & 60 \\
\hline $10 \times 10=$ & 100 & $3 \times 10=$ & 30 & $5 \times 11=$ & 55 \\
\hline $16 \times 11=$ & 176 & $5 \times 11=$ & 55 & $4 \times 12=$ & 48 \\
\hline $21 \times 12=$ & 252 & $4 \times 12=$ & 48 & & \\
\hline & 830 & & 233 & $21 \div 3=$ & 7 \\
\hline $15 \div 3=$ & 5 & $18 \div 3=$ & 6 & Contents & \\
\hline Contents, & 4,150 & Contents, & 1,398 & & \\
\hline $4 \times 6=$ & 24 & $7 \times 6=$ & 42 & $7 \times 8=$ & 56 \\
\hline $7 \times 7=$ & 49 & $4 \times 7=$ & 28 & $7 \times 9=$ & 63 \\
\hline $4 \times 8=$ & 32 & $4 \times 8=$ & 32 & $9 \times 10=$ & 90 \\
\hline $5 \times 9=$ & 45 & $4 \times 9=$ & 36 & $12 \times 11=$ & 132 \\
\hline $5 \times 10=$ & 50 & $10 \times 10=$ & 100 & $11 \times 12=$ & 132 \\
\hline $4 \times 11=$ & 44 & $5 \times 12=$ & 60 & & \\
\hline $12 \times 13=$ & 156 & \multirow{4}{*}{$\begin{array}{l}19 \div 3= \\
\text { Contents, }\end{array}$} & & \multirow{4}{*}{$\begin{array}{l}22 \div 3= \\
\text { Contents, }\end{array}$} & \multirow{2}{*}{$\begin{array}{r}515 \\
7 \frac{1}{3}\end{array}$} \\
\hline & 400 & & $6 \frac{1}{3}$ & & \\
\hline $16 \div 3=$ & $5 \frac{1}{3}$ & & & & 3,777 \\
\hline Contents, & 2,133 & & & & \\
\hline
\end{tabular}


Solution of Specification No. 3.-(Continued.)

\begin{tabular}{|c|c|c|c|c|}
\hline $\mathrm{Br}$. & Products. & No. & Products. & \multirow{10}{*}{$\begin{array}{l}24 \text { feet being the } \\
\text { length of the pieces } \\
\text { in the last column, } \\
\text { I take the } \frac{1}{3} \text { of it } \\
8 \text {, and multiply it } \\
\text { by the product of } \\
\text { the No. of pieces } \\
\text { and their breadths. }\end{array}$} \\
\hline $8 \times 6=$ & 48 & $6 \times 6=$ & 36 & \\
\hline $3 \times 7=$ & 21 & $4 \times 7=$ & 28 & \\
\hline $2 \times 8=$ & 16 & $8=$ & 40 & \\
\hline $3 \times 9=$ & 27 & $3 \times 9=$ & 27 & \\
\hline $1 \times 10=$ & 10 & $3 \times 10=$ & 30 & \\
\hline $2 \times 11=$ & 22 & $11 \times 11=$ & 121 & \\
\hline $3 \times 12=$ & 36 & $6 \times 12=$ & 72 & \\
\hline$-3=$ & $\begin{array}{r}180 \\
72\end{array}$ & \multicolumn{2}{|l|}{$24 \div 3$} & \\
\hline Contents, & 1,380 & Contents, & 2,832 & \\
\hline
\end{tabular}

Rule for computing 5-inch Timber.

Multiply the number of pieces in each square of the shingle, by their width as given in the top column, and the product by the length divided by $2 \frac{2}{5}$ for the contents.

By multiplying the length of a 5 -inch stick by the width of the same, and the product by the length divided by $2 \frac{2}{5}$, you will get the contents in feet of Board Measure.

Required the contents of 33 pieces 10 feet long of 5 $\times 5$.

1 st Solution. $-33 \times 10=330 \times 2 \frac{1}{12}=687 \frac{1}{2}$ feet.

$2 d$ Solution. - Find the contents of 10 pieces 33 feet long and 5 by $5.10 \times 5=50,33 \div 2 \frac{2}{5}=\frac{5}{12} \times \frac{33}{1}=$ $\frac{18}{12}=13 \frac{3}{4}$, therefore $50 \times 13 \frac{3}{4}=687 \frac{1}{2}=$ Ans. 
ON LUMBER SURVEYING.

Timber Shingle Five-inch, No. 4.

\begin{tabular}{|c|c|c|c|c|c|c|c|c|}
\hline 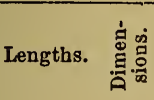 & $\begin{array}{l}10 \\
X \\
20\end{array}$ & $\begin{array}{l}6 \\
X \\
10\end{array}$ & $\underset{10}{x}$ & $\begin{array}{c}\infty \\
X \\
10\end{array}$ & $\begin{array}{l}0 \\
x\end{array}$ & $\begin{array}{l}0 \\
\times \\
10\end{array}$ & $\begin{array}{l}\bar{X} \\
\bar{x}\end{array}$ & $\begin{array}{l}\mathcal{N} \\
\times \\
x\end{array}$ \\
\hline 20 & …… & $\begin{array}{ll}* & \\
& 2\end{array}$ & $\cdots$ & $\mid \begin{array}{r}\cdots . . .0 \\
16\end{array}$ & $\begin{array}{ll}* & \\
& 2\end{array}$ & - 1 & $\cdots{ }_{5}$ & $\begin{array}{ll}\cdots \cdots & \\
& 5\end{array}$ \\
\hline 21 & ......... & $\cdots{ }_{6}$ & $\cdots$ & $\mid \begin{array}{r}\cdots \ldots . . . \\
8\end{array}$ & $\cdots{ }_{5}$ & $\cdots \cdots{ }_{5}$ & $\cdots{ }_{4}$ & $\cdots$ \\
\hline 22 & $\cdots \cdots{ }_{6}$ & $\cdots{ }_{5}$ & $\cdots{ }_{5}$ & $\begin{array}{ll}\bullet^{\prime} & \\
& 2\end{array} \mid$ & $\cdots_{3}$ & $\cdots{ }_{5}$ & $\cdots ._{5}$ & $\begin{array}{ll}\cdots & \\
& 5\end{array}$ \\
\hline 23 & & $\cdots{ }_{6}$ & $\begin{array}{ll}{ }^{*} & 2\end{array}$ & $\cdots{ }_{5}$ & $\cdots{ }_{5}$ & $\begin{array}{r}\cdots . . . . \\
8\end{array}$ & $\begin{array}{ll}\cdot \bullet & \\
& 2\end{array} \mid$ & $\cdots \cdots$ \\
\hline 24 & $\cdots$ & & $\cdots_{3}$ & $\begin{array}{r}\ldots . . . . . . . \\
8\end{array}$ & ${ }^{\cdots} \quad 3$ & 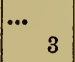 & 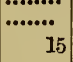 & $\cdots$ \\
\hline 25 & $\ddot{*}_{2}$ & 1 & 1 & $\begin{array}{ll}\cdot \cdots & \\
& 2\end{array} \mid$ & $\cdots_{4}$ & & $\cdots_{4}$ & $\cdots$ \\
\hline 26 & 7 & $\begin{array}{ll}\cdots & \\
& 3\end{array}$ & ${ }^{*} \quad 2$ & 1 & $\cdots{ }_{4}$ & $\cdots_{4}$ & $\begin{array}{ll}{ }^{*} & \\
& 2\end{array} \mid$ & 1 \\
\hline 30 & & ……. 12 & $\cdots$ & $\begin{array}{ll}\cdots & \\
& 3\end{array} \mid$ & $\cdots_{3}$ & $\begin{array}{cc}\cdots & \ldots \\
\because & 10\end{array}$ & $\begin{array}{ll}\cdots_{3} & \\
3\end{array}$ & ." \\
\hline 31 & $\begin{array}{r}\cdots \cdot . \cdot . \\
7\end{array}$ & $\cdots$ & $\begin{array}{ll}\cdots & \\
& \end{array}$ & $\begin{array}{ll}\cdots & \\
3\end{array} \mid$ & $\cdots_{3}$ & $\begin{array}{ll}\cdots^{\prime} & \\
3\end{array} \mid$ & $\cdots \cdots$ & $\because \cdots$ \\
\hline 32 & & $\begin{array}{r}7.6 . \\
7\end{array}$ & $\cdots_{3}$ & $\begin{array}{ll}\because & \\
& 2\end{array} \mid$ & $\cdots_{4}$ & $\begin{array}{ll}\cdots & \\
\cdots\end{array} \mid$ & $\begin{array}{ll}{ }^{\prime} & 2\end{array}$ & $\cdot$ \\
\hline 33 & $\because \cdots 10$ & & 2 & 1 & $\begin{array}{ll}\cdots & \\
& 3\end{array}$ & $\begin{array}{ll}\cdots^{\prime} & \\
& 3\end{array} \mid$ & $\cdots{ }_{5}$ & $\cdots$ \\
\hline
\end{tabular}


Specification of Five-inch Timber Shingle, No. 4.

\begin{tabular}{|c|c|c|c|c|c|c|c|c|c|c|}
\hline Lengths. & 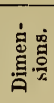 & ${ }_{10}^{10} X$ & $\begin{array}{l}0 \\
X \\
10\end{array}$ & ${ }_{20}^{1}$ & $\left|\begin{array}{l}\infty \\
X \\
10\end{array}\right|$ & $\begin{array}{l}0 \\
X \\
0\end{array} \mid$ & $\left|\begin{array}{l}0 \\
X \\
10\end{array}\right|$ & $\begin{array}{l}E \\
X \\
10\end{array}$ & $\begin{array}{l}\stackrel{N}{7} \\
X \\
10\end{array}$ & Contents. \\
\hline 20 & & 7 & 2 & 5 & 16 & 2 & 1 & 5 & 5 & 2,941 \\
\hline 21 & & 8 & 6 & 5 & 8 & 5 & 5 & 4 & 4 & 3,167 \\
\hline 22 & & 6 & 5 & 5 & 2 & 3 & 5 & 5 & 5 & 2,778 \\
\hline 23 & & & 6 & 2 & 5 & 5 & 8 & 2 & 8 & 3,191 \\
\hline 24 & & 20 & 7 & 3 & 8 & 3 & 3 & 15 & 9 & 5,570 \\
\hline 25 & & 2 & 1 & 1 & 2 & 4 & & 4 & 5 & 1,865 \\
\hline 26 & & 7 & 3 & 2 & 1 & 4 & 4 & 2 & 1 & 2,004 \\
\hline 30 & & & 12 & 6 & 3 & 3 & 10 & 3 & 2 & 4,025 \\
\hline 31 & & 7 & 5 & 3 & 3 & 3 & 3 & 6 & 9 & 4,405 \\
\hline 32 & & & 7 & 3 & 2 & 4 & 3 & 2 & 1 & 2,387 \\
\hline \multirow[t]{2}{*}{33} & & 10 & 4 & 2 & 1 & 3 & 3 & 5 & 9 & 4,345 \\
\hline & & & & & & & & & & Total, 36,678 feet. \\
\hline
\end{tabular}

Example, showing how to compute a 5-inch Specification.

\begin{tabular}{|c|c|c|c|}
\hline $\mathrm{Br}$. & $\mathrm{Br}$. & No. & No. \\
\hline$\times 5=35$ & $8 \times 5=40$ & $6 \times 5=30$ & $6 \times 6=36$ \\
\hline$\times 6=12$ & $6 \times 6=36$ & $5 \times 6=30$ & $2 \times 7=14$ \\
\hline $5 \times 7=35$ & $5 \times 7=35$ & $5 \times 7=35$ & $5 \times 8=40$ \\
\hline $8 \times 16=128$ & $8 \times 8=64$ & $2 \times 8=16$ & $5 \times 9=45$ \\
\hline$\times 9=18$ & $5 \times 9=45$ & $3 \times 9=27$ & $8 \times 10=80$ \\
\hline$\times 10=10$ & $5 \times 10=50$ & $5 \times 10=50$ & $2 \times 11=22$ \\
\hline$\times 11=55$ & $4 \times 1$ & $5 \times 11=55$ & $8 \times 12=96$ \\
\hline $5 \times 12=60$ & $4 \times 12=48$ & $=60$ & \\
\hline$*_{20} \div 2 \frac{22}{5}=8 \frac{1}{3}$ & $21 \div 2 \frac{2}{5}=8 \frac{3}{4}$ & $\begin{array}{r}303 \\
22 \div 2 \frac{2}{5}=9 \frac{1}{6}\end{array}$ & $\begin{array}{c}333 \\
-2 \frac{2}{5}=9 \frac{7}{12}\end{array}$ \\
\hline Contents, & Contents, $\mathbf{3 , 1 6 7}$ & Contents, 2,778 & Contents, 3,191 \\
\hline
\end{tabular}

* 20 feet, the length of the pieces, divided by $2 \frac{2}{5}$, and the result, $8 \frac{1}{3}$, multiplied by $353=2,941$ feet $=$ contents of $20 \mathrm{ft}$ pieces.

$$
\text { Invert } \frac{12}{5}=\frac{5}{12} \times \frac{20}{1}=\frac{100}{12}=8 \frac{1}{3}
$$


ON LUMBER SURVEYING.

Timber Shingle, Six-inch, No. 5.

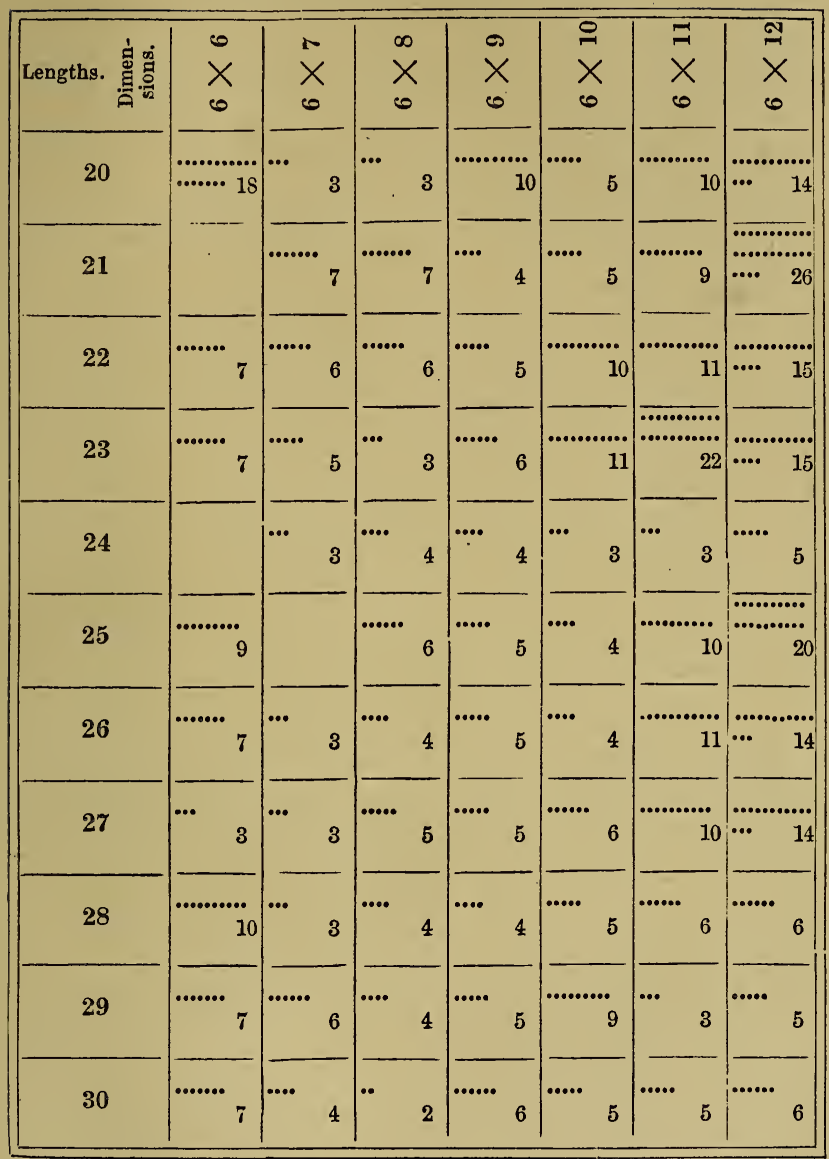

Rule for finding the Contents of 6-inch Timber.

Multiply the number of pieces or dots by the width of said pieces, and then multiply the product by half the length of one of the pieces, for the contents. 
What are the contents of 18 pieces of $6 \times 6$, and 20 feet long? $18 \times 6=108 ; 20 \div 2=10,108 \times 10=1,080$ feet. By the Table $6 \times 6$ is three times the length for the contents, therefore $20 \times 18=360$ feet running length, 360 feet $\times 3$ feet $=1,080$. Ans. 1,080.

So we find the same result by both rules.

Specification of Timber Shingle, No. 5.

\begin{tabular}{|c|c|c|c|c|c|c|c|c|c|}
\hline Lengths. & 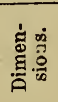 & $\begin{array}{l}0 \\
X \\
0\end{array} \mid$ & $\begin{array}{c}x \\
x\end{array}$ & $\begin{array}{c}\infty \\
X \\
0\end{array} \mid$ & $\begin{array}{l}\infty \\
X \\
0\end{array}$ & $\begin{array}{l}0 \\
\times \\
0\end{array}$ & $\begin{array}{l}\overline{-1} \\
X \\
0\end{array}$ & $\begin{array}{l}\underset{N}{N} \\
X \\
0\end{array}$ & Contents. \\
\hline 20 & & 18 & 3 & 3 & 10 & 5 & 10 & 14 & 5,710 \\
\hline 21 & & & 7 & 7 & 4 & 5 & 9 & 26 & 6,321 \\
\hline 22 & & 7 & 6 & 6 & 5 & 10 & 11 & 15 & 6,358 \\
\hline 23 & & 7 & 5 & 3 & 6 & 11 & 22 & 15 & 7,900 \\
\hline 24 & & & 3 & 4 & 4 & 3 & 3 & 5 & 2,544 \\
\hline 25 & & 9 & & 6 & 5 & 4 & 10 & 20 & 6,712 \\
\hline 26 & & 7 & 3 & 4 & 5) & 4 & 11 & 14 & 6,097 \\
\hline 27 & & 3 & 3 & 5 & 5 & 6 & 10 & 14 & 6,237 \\
\hline 28 & & 10 & 3 & 4 & 4 & 5 & 6 & 6 & 4,718 \\
\hline 29 & & 7 & 6 & 4 & 5 & 9 & 3 & 5 & 4,988 \\
\hline 30 & & 7 & 4 & 2 & 6 & 5 & 5 & 6 & 4,755 \\
\hline & & & & & & & & & Total, 62,340 feet. \\
\hline
\end{tabular}

Examples showing how to compute the Specification No. 5 of 6-inch Timber.

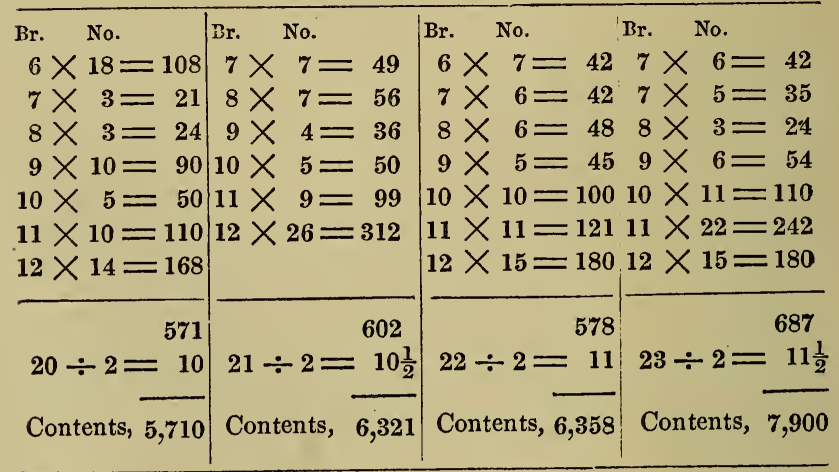


What is the cost of a piece of pine timber 6 inches $\times 10$ inches, and 38 feet in length @ $3 \frac{1}{2}$ cts. per foot?

Ans. $\$ 6.65$.

Solution. - Length $38 \div 2=19 ; 19 \times$ by the breadth $10=190$ feet, contents. 190 feet $@ 3 \frac{1}{2}=\$ 6.65$.

By the Second Rule. 6 inches $\times 10$ inches $=5$ times the length, for the contents, therefore $38 \times 5=190$ feet. 190 feet $\times 3 \frac{1}{2}$ cts. $=\$ 6.65$.

Rule for finding the Contents of 7-inch Timber.

Multiply the width by the length, divided by $1 \frac{5}{7}$.

Required the contents of a piece of timber $7 \times 7$ and 20 feet long?

Divide the length, 20 feet, by $1 \frac{5}{7}\left(20 \div 1 \frac{5}{7}=11 \frac{2}{3}\right)$, and multiply the breadth, 7 inches, by the quotient, $11 \frac{2}{3}$.

$11 \frac{2}{3}=\frac{35}{3} ; \frac{35}{3} \times \frac{7}{1}=2 \frac{45}{3}=81 \frac{2}{3}$ feet $=$ contents in superficial feet.

$2 \mathrm{~d}$ Operation. - By the table $7 \times 7$ is $=$ to $4_{1 \frac{1}{2}}$ times the length, for the contents, therefore 20 feet $\times 4_{\frac{1}{2}}^{\frac{1}{2}}=81 \frac{2}{3}$ feet $=$ contents.

Timber is often surveyed and the contents marked on each piece, and then put down on a shingle for contents in its proper column. 
Timber Shingle, Seven-inch. No. 6, and Specification.

\begin{tabular}{|c|c|c|c|c|c|c|c|}
\hline 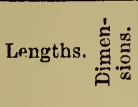 & $\frac{n}{x}$ & $\underset{n}{\infty}$ & $\frac{1}{x}$ & $\stackrel{0}{x}$ & $\begin{array}{l}E \\
x \\
n\end{array}$ & $\underset{n}{\mathfrak{n}}$ & Contents. \\
\hline 20 & $\begin{array}{r}\bullet \cdots \ldots \ldots . . . \\
\cdots \ldots \ldots \ldots \\
\quad 24 \\
\end{array}$ & $\begin{array}{r}\ldots . . . . . . . \\
\cdots . . . \\
\quad 16 \\
\end{array}$ & $\begin{array}{r}\cdots \cdots \cdot \\
\quad 7 \\
\end{array}$ & $\begin{array}{r}\cdots \cdot . \\
\quad 5 \\
\end{array}$ & $\begin{array}{r}\cdots \cdots . . . . . \\
18 \\
\end{array}$ & $\begin{array}{r}\cdots \cdots . . . \\
\quad \cdots . . . \\
\end{array}$ & 9,321 \\
\hline 21 & $\begin{array}{r}\ldots . . . . . . \\
\quad 9 \\
\end{array}$ & $\begin{array}{r}\cdots . . . \\
6 \\
\end{array}$ & $\begin{array}{r}\cdots . . . \\
8 \\
\end{array}$ & ...... & $\begin{array}{r}\cdots . . . . . \\
8\end{array}$ & $\begin{array}{r}\cdots \cdot . . \\
6\end{array}$ & 5,059 \\
\hline 22 & $\cdots$ & 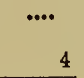 & $\cdots$ & & ........ & 8 & 10,062 \\
\hline 23 & $\begin{array}{r}\cdots \cdots \cdots . . . \\
\quad 9 \\
\end{array}$ & $\begin{array}{l}\cdots \\
4\end{array}$ & $\begin{array}{r}\cdots \cdots . . \\
7 \\
\end{array}$ & $\begin{array}{r}\cdots \cdots \cdot . . \\
7\end{array}$ & $\begin{array}{r}\ldots . . . . . . . . . \\
18 \\
\end{array}$ & $\begin{array}{r}0 \ldots \ldots \ldots . . \\
\cdots \ldots \ldots \ldots . . \\
27 \\
\end{array}$ & 10,062 \\
\hline 24 & ….... & ...... & ....... & $\begin{array}{r}\cdots \cdots \cdot \\
6\end{array}$ & $\begin{array}{r}\cdots \ldots \ldots . . \\
9\end{array}$ & $\begin{array}{r}\cdots \cdots \cdots \\
8\end{array}$ & 7,420 \\
\hline 25 & & $\begin{array}{l}\cdots \\
\quad 4 \\
\end{array}$ & $\begin{array}{r}\cdots \cdot . \\
5 \\
\end{array}$ & $\begin{array}{r}\cdots . . .6 \\
6 \\
\end{array}$ & $\begin{array}{r}\cdots \cdot . . \\
6 \\
\end{array}$ & $\begin{array}{l}\cdots \\
\quad 4 \\
\end{array}$ & 3,807 \\
\hline 26 & $\begin{array}{r}\ldots . . . . . \\
\cdots \cdots . . . \\
14 \\
\end{array}$ & $\begin{array}{l}\cdot . \\
\quad 2 \\
\end{array}$ & & $\begin{array}{r}\cdots . . . \\
6 \\
\end{array}$ & .... & $\cdots$ & 1,493 \\
\hline 27 & $\begin{array}{r}\cdots \cdot . . \\
5 \\
\end{array}$ & ….. & $\begin{array}{c}\cdots . . \\
4 \\
\end{array}$ & $\begin{array}{r}\cdots \cdot . \\
5 \\
\end{array}$ & $\begin{array}{r}\cdots \cdots . . . \\
7\end{array}$ & $\begin{array}{r}\cdots . . . . \\
7 \\
\end{array}$ & 5,197 \\
\hline 28 & $\begin{array}{r}\cdots . . . . . \\
8 \\
\end{array}$ & $\begin{array}{c}\cdots \\
\quad 4 \\
\end{array}$ & $\begin{array}{r}\cdots . . \\
4 \\
\end{array}$ & $\begin{array}{r}\cdots . . . \\
7 \\
\end{array}$ & $\begin{array}{r}\ldots . . . . . \\
8 \\
\end{array}$ & .... & 5,390 \\
\hline 29 & $\begin{array}{r}\ldots . . . . . . \\
15 \\
\end{array}$ & $\begin{array}{r}\cdots \cdot . . . \\
7 \\
\end{array}$ & $\begin{array}{r}\cdots \cdot . . \\
7 \\
\end{array}$ & $\begin{array}{l}\cdots \\
\quad 4 \\
\end{array}$ & $\begin{array}{r}\cdots . . . \\
\quad 6 \\
\end{array}$ & $\begin{array}{l}\bullet \\
2 \\
\end{array}$ & 5,988 \\
\hline 30 & $\begin{array}{r}\cdots . . . . \\
8 \\
\end{array}$ & $\begin{array}{l}\cdots . . \\
\quad 4 \\
\end{array}$ & $\begin{array}{r}\cdots . . . \\
5 \\
\end{array}$ & $\begin{array}{r}. . . . . . . \\
8 \\
\end{array}$ & $\begin{array}{r}\quad . . . . . \\
7 \\
\end{array}$ & $\begin{array}{r}\ldots . . . . \\
8 \\
\end{array}$ & 6,755 \\
\hline 31 & $\begin{array}{r}\cdots . . . . . . \\
16\end{array}$ & $\begin{array}{c}\ldots . . . . \\
12 . \\
\end{array}$ & $\cdots$ & $\begin{array}{r}\bullet \\
\end{array}$ & $\begin{array}{ll}\cdot & \\
1 & 1 \\
\end{array}$ & $\begin{array}{ll}\cdots \\
3\end{array}$ & 5,624 \\
\hline
\end{tabular}


ON LUMBER SURVEYING.

Timber Shingle, Eight-inch. No. 7.

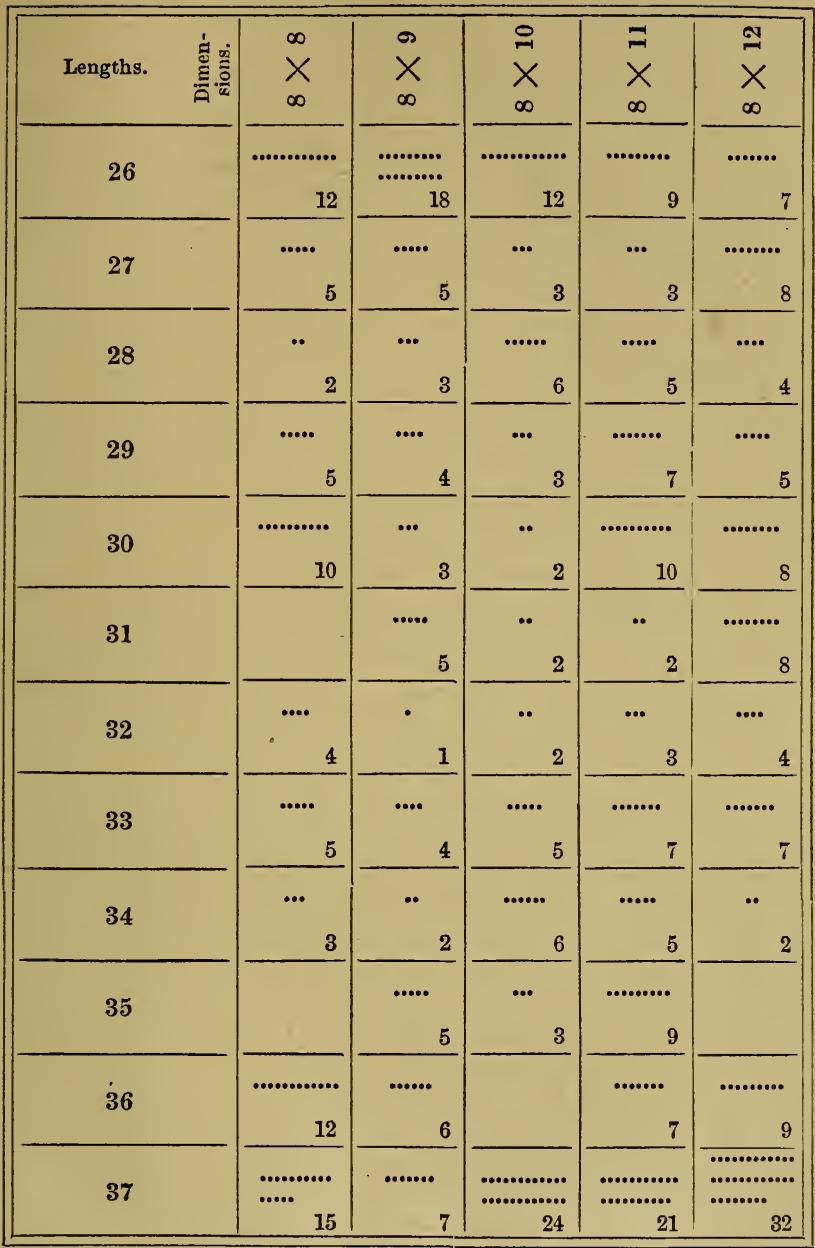

Rule for finding the Contents of 8 by 8 Timber.

Divide the length by $1 \frac{1}{2}$, and multiply the quotient by the width of the timber for the contents in feet of board measure. 
ExaMPLE showing how the first column of 8-inch specification is done.

Br. No. pieces each 26 feet long.

$8 \times 12=96$

$9 \times 18=162$

$10 \times 12=120$

$11 \times 9=99$

$12 \times 7=84$

$26 \div 1 \frac{1}{2}=\frac{\overline{561}}{\frac{14}{2244}}$
$26 \div 1 \frac{1}{2}=\frac{3}{2}$.

Invert the divisor, $\frac{2}{3} \times{ }_{1}^{26}=\frac{52}{3}=14$.

\section{4 feet $=$ contents.}

Specification Shingle, Eight-inch. No. 7.

\begin{tabular}{|c|c|c|c|c|c|c|}
\hline Lengths. & $\begin{array}{l}\infty \\
X \\
\infty\end{array}$ & $\begin{array}{l}\infty \\
x \\
\infty\end{array}$ & $\begin{array}{l}\stackrel{ }{X} \\
\underset{\infty}{X}\end{array}$ & $\begin{array}{l}\exists \\
\bar{\infty}\end{array}$ & $\begin{array}{l}\stackrel{N}{\sim} \\
\underset{\infty}{X}\end{array}$ & Contents. \\
\hline 26 & 12 & 18 & 12 & 9 & 7 & 7,854 \\
\hline 27 & 5 & 5 & 3 & 3 & 8 & 4,392 \\
\hline 28 & 2 & 3 & 6 & 5 & 4 & 3,845 \\
\hline 29 & 5 & 4 & 3 & 7 & 5 & 4,698 \\
\hline 30 & 10 & 3 & 2 & 10 & 8 & 6,660 \\
\hline 31 & & 5 & 2 & 2 & 8 & 3,782 \\
\hline 32 & 4 & 1 & 2 & 3 & 4 & 3,029 \\
\hline 33 & 5 & 4 & 5 & 7 & 7 & 6,314 \\
\hline 34 & 3 & 2 & 6 & 5 & 2 & 4,103 \\
\hline 35 & & 5 & 3 & 9 & & 4,060 \\
\hline 36 & 12 & 6 & & 7 & 9 & 8,040 \\
\hline \multirow[t]{2}{*}{37} & 15 & 7 & 24 & 21 & 32 & 38,406 \\
\hline & & & & & \multicolumn{2}{|c|}{ Total, $9,5183 \mathrm{fe}$} \\
\hline
\end{tabular}


ON LUMBER SURVEYING.

Timber Shingle, Nine-inch. No. 8.

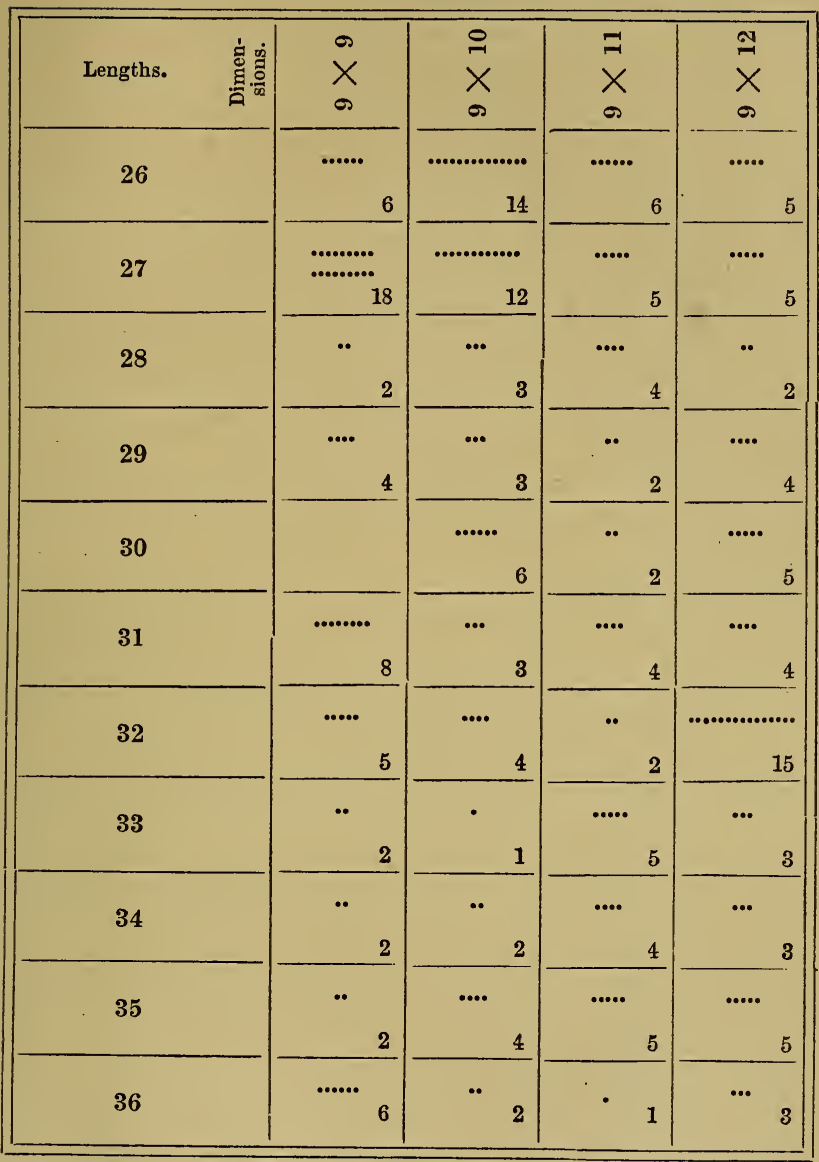

Rule for finding the Contents of Nine-inch Timber. Divide the length by $1 \frac{1}{3}$ and multiply the quotient by the breadth of the stick for the contents.

Required the contents of a piece of timber $9 \times 12$ inches and 26 feet long?

$26 \div 1 \frac{1}{3}=19 \frac{1}{2} . \quad 19 \frac{1}{2} \times 12=234=$ contents. 
Timber Shingle, Ten-inch. No. 9.

\begin{tabular}{|c|c|c|c|c|}
\hline Lengths. & : & $10 \times 10$ & $10 \times 11$ & $10 \times 12$ \\
\hline 26 & & 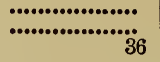 & 13 & $\cdots$ \\
\hline 27 & & $\begin{array}{ll}\cdots & \\
& 4 \\
\end{array}$ & $\cdots$ & $\cdots$ \\
\hline 28 & & 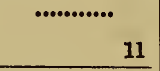 & $\ldots .$. & 11 \\
\hline 29 & & $\begin{array}{l}\cdots . . . . . . \\
\\
\end{array}$ & $\bullet$ & $\cdots$ \\
\hline 30 & & $\cdots$ & $\ldots . .$. & $\cdots$ \\
\hline 31 & & ...... & $\bullet$ & - \\
\hline 32 & & 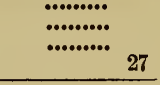 & $\cdots$ & $\cdots$ \\
\hline 33 & & $\cdots$ & $\cdots$ & $\cdots$ \\
\hline 34 & & $\cdots$ & $\bullet$ & $\cdots \cdots$ \\
\hline 35 & & - 1 & $\bullet$ & $\cdots$ \\
\hline 36 & & ............ 12 & ….............. & 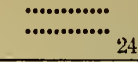 \\
\hline
\end{tabular}


ON LUMBER SURVEYING.

Specification of Timber Shingle, Nine-inch. No. 8.

\begin{tabular}{|c|c|c|c|c|c|}
\hline Lengths. & $\begin{array}{l}\dot{\theta} \\
\dot{x}\end{array}$ & $\begin{array}{l}0 \\
\times \\
0\end{array}$ & $\begin{array}{l}z \\
\bar{x} \\
0\end{array}$ & $\underset{0}{\stackrel{N}{X}}$ & Contents. \\
\hline 26 & 6 & 14 & 6 & 5 & 6,240 \\
\hline 27 & 18 & 12 & 5 & 5 & 8,039 \\
\hline 28 & 2 & 3 & 4 & 2 & 2,436 \\
\hline 29 & 4 & 3 & 2 & 4 & 2,958 \\
\hline 30 & & 6 & 2 & 5 & 3,195 \\
\hline 31 & 8 & 3 & 4 & 4 & 4,510 \\
\hline 32 & 5 & 4 & 2 & 15 & 6,888 \\
\hline 33 & 2 & 1 & 5 & 3 & 2,945 \\
\hline 34 & 2 & 2 & 4 & 3 & 3,009 \\
\hline 35 & 2 & 4 & 5 & 5 & 4,541 \\
\hline \multirow[t]{2}{*}{36} & 6 & 2 & 1 & 3 & 3,267 \\
\hline & & & & & Contents, 48,028 feet. \\
\hline
\end{tabular}

Specification of Timber Shingle, Ten-inch. No. 9.

\begin{tabular}{|c|c|c|c|c|}
\hline Lengths. & $\begin{array}{l}\stackrel{0}{-} \\
\times \\
\end{array}$ & $\begin{array}{l}\beth \\
\times \\
\varrho\end{array}$ & 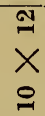 & Contents. \\
\hline 26 & 36 & 13 & 5 & 12,198 \\
\hline 27 & 4 & 5 & 4 & 3,297 \\
\hline 28 & 11 & 5 & 11 & 6,930 \\
\hline 29 & 8 & 3 & 4 & 3,891 \\
\hline 30 & 4 & 6 & 4 & 3,850 \\
\hline 31 & 6 & 2 & 1 & 2,428 \\
\hline 32 & 27 & 3 & 3 & 9,040 \\
\hline 33 & 5 & 5 & 5 & 4,587 \\
\hline 34 & 3 & 2 & 6 & 3,513 \\
\hline 35 & 1 & 2 & 5 & 2,683 \\
\hline \multirow[t]{2}{*}{36} & 12 & 25 & 24 & 20,490 \\
\hline & & & & Contents, $\mathbf{7 2 , 9 0 7}$ feet. \\
\hline
\end{tabular}




\section{Rule for Ten-inch Timber.}

Divide the length by $1 \frac{1}{5}$ and multiply the quotient by the breadth, for the contents in feet of board measure.

Required the contents of a stick 36 feet long 10 inches by 11 inches?

$36 \div 1 \frac{1}{5}=30$, and $30 \times 11=330$ feet $=$ contents.

$2 d$ Solution. - By the table $10 \times 11$ is $9 \frac{1}{6}$ times the length, for the contents; therefore, 36 feet $\times 9 \frac{1}{6}=330$ feet $=$ contents.

Fxamples showing how 9 and 10 inch specifications are made out.

Nine-inch.

Br. Pieces. Pro.

$9 \times 6=54$

$10 \times 14=140$

$11 \times 6=66$

$12 \times 5=60$

$\overline{320}$

$26 \div 1 \frac{1}{3}=19 \frac{1}{2}$

$\overline{2880}$

320

160

Contents $=6240$

Length, $26 \div 1 \frac{1}{3} ; 1 \frac{1}{3}=$

$\frac{4}{3}$. Inverted $=\frac{3}{4} ; \frac{3}{4} \times \frac{26}{1}$

$=\frac{78}{4}=19 \frac{1}{2}$.

P. S. - All the specifications in this book are done in a manner similar to the specification of the Plank Shingle No. 1.

\section{Ten-inch.}

Br. Pieces. Pro.

$10 \times 36=360$

$11 \times 13=143$

$12 \times 5=60$

563

2

3) 1126

375

563

563

1126

375
$21 \frac{2}{3}$

12,198 feet

Length, $26 \div 1 \frac{1}{5} ; 1 \frac{1}{5}=$ $\frac{6}{5}=$ inverted to $\frac{5}{6} ; \frac{5}{6} \times{ }_{1}^{2,6}$ $=1 \frac{3}{6} \underline{0}=21 \frac{2}{3}$. 
ON LUMBER SURVEYING.

Eleven-inch Shingle No. 10.

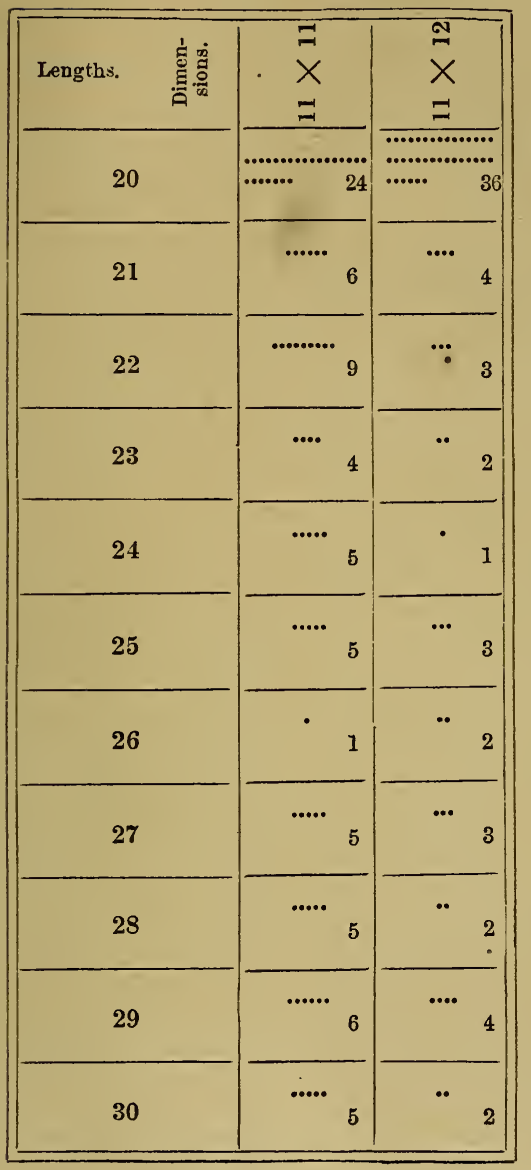

હூ

률

¿

ह

$\sim$ है

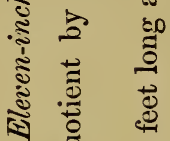

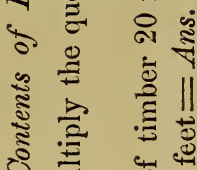

ह ह गे

일 है।

त म ब त

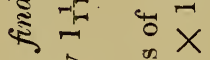

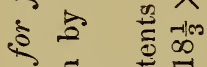

ॠ

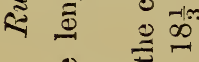

$\cong \dot{0}$

을

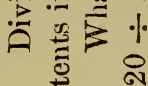


SELF-INSTRUCTOR

Timber Shingle, Twelve-inch, No. 11.

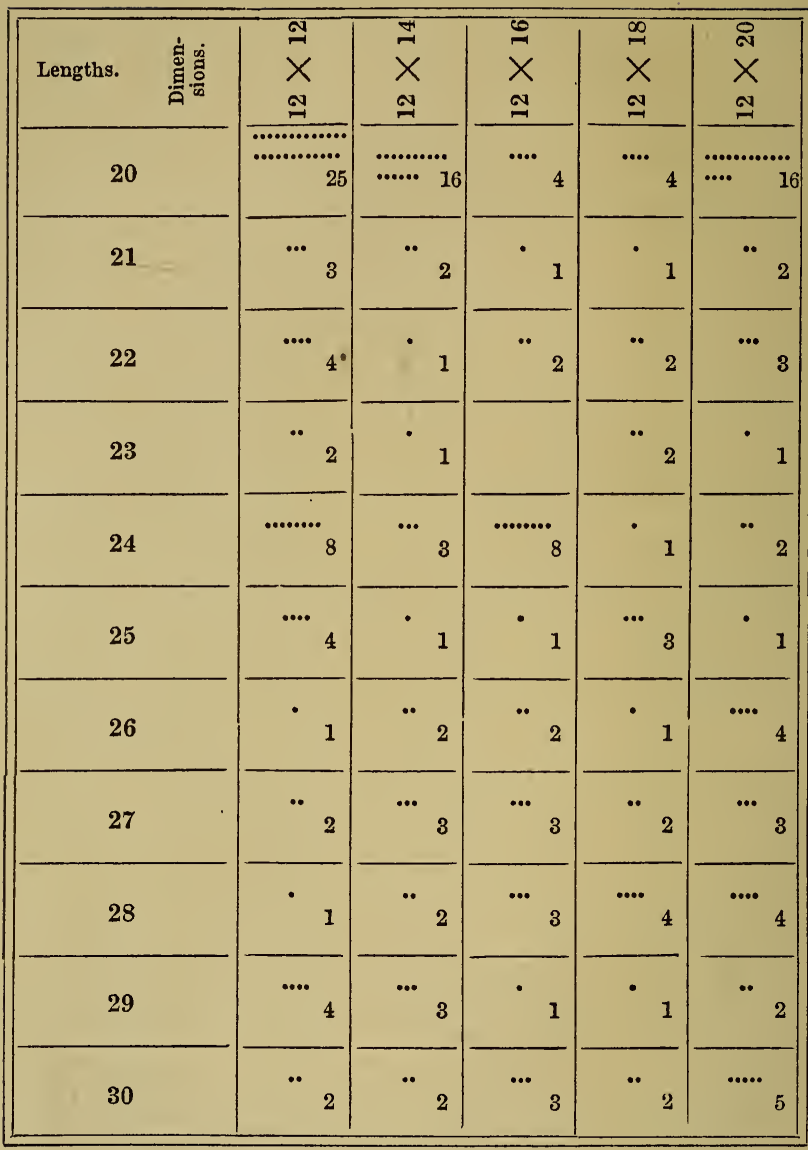

Rule for Twelve-inch Timber.

Multiply the length by the width for the contents in feet. Required, the contents of 16 pieces of $12 \times 20$ inch timber, and 20 feet long? $16 \times 20=320 . \quad 320 \times 20=6,400$ feet $=$ contents in feet of board measure. 
ON LUMBER SURVEYING.

Specification of Shingle No. 10.

\begin{tabular}{|c|c|c|c|}
\hline Lengths. 苛递 & $11 \times 11$ & $11 \times 12$ & Contents. \\
\hline 20 & 24 & 36 & 12,760 \\
\hline 21 & 6 & 4 & 2,194 \\
\hline 22 & 9 & 3 & 2,722 \\
\hline 23 & 4 & 2 & 1,434 \\
\hline 24 & 5 & 1 & 1,774 \\
\hline 25 & 5 & 3 & 2,085 \\
\hline 26 & 1 & 2 & 834 \\
\hline 27 & 5 & 3 & 2,252 \\
\hline 28 & 5 & 2 & 2,028 \\
\hline 29 & 6 & 4 & 3,030 \\
\hline 30 & 5 & 2 & 2,272 \\
\hline & & \multicolumn{2}{|c|}{ Total, 33,285 } \\
\hline
\end{tabular}

Specification of Shingle No. 11.

\begin{tabular}{|c|c|c|c|c|c|c|}
\hline Lengths. & 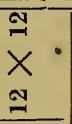 & $\begin{array}{l}\vec{z} \\
X \\
\mathcal{I}\end{array}$ & $\begin{array}{l}0 \\
\stackrel{0}{X} \\
\stackrel{N}{\sim} \\
\end{array}$ & $\begin{array}{l}\underset{\infty}{\sim} \\
\times \\
\stackrel{\sim}{\sim} \\
\end{array}$ & $\begin{array}{l}\text { iิ } \\
\times \\
\text { ஸ् }\end{array}$ & Contents. \\
\hline 20 & 25 & 16 & 4 & 4 & 16 & 19,600 \\
\hline 21 & 3 & 2 & 1 & 1 & 2 & 2,898 \\
\hline 22 & 4 & 1 & 2 & 2 & 3 & 4.180 \\
\hline 23 & 2 & 1 & & 2 & 1 & 2,162 \\
\hline 24 & 8 & 3 & 8 & 1 & 2 & 7,776 \\
\hline 25 & 4 & 1 & 1 & 3 & 1 & 3,800 \\
\hline 26 & 1 & 2 & 2 & 1 & 4 & 4,420 \\
\hline 27 & 2 & 3 & 3 & 2 & 3 & 5.670 \\
\hline 28 & 1 & 2 & 3 & 4 & 4 & 6,720 \\
\hline 29 & 4 & 3 & 1 & 1 & 2 & 4,756 \\
\hline 30 & 2 & 2 & 3 & 2 & 5 & 7,080 \\
\hline & & & & & \multicolumn{2}{|c|}{ Total, 69,071} \\
\hline
\end{tabular}


Rule for finding the Contents, of Battens or Two-and-aHalf-inch Stuff.

Inch.

$2 \frac{1}{2} \times 2=\frac{5}{12}$

$2 \frac{1}{2} \times 3=\frac{5}{8}$

$2 \frac{1}{2} \times 4=\frac{5}{6}$

$2 \frac{1}{2} \times 5=1 \frac{1}{24}$

$2 \frac{1}{2} \times 6=1 \frac{1}{4}$

$2 \frac{1}{2} \times 7=1 \frac{11}{24}$

\begin{tabular}{|l|l} 
Inch. & What are the contents \\
$2 \frac{1}{2} \times 8=1 \frac{2}{3}$ & of a batten $22 \mathrm{ft.l}$ long $2 \frac{1}{2}$ \\
$2 \frac{1}{2} \times 9=1 \frac{7}{8}$ & inches by 12 inches? \\
$2 \frac{1}{2} \times 10=2 \frac{1}{12}$ & By this rule $2 \frac{1}{2} \times 12$ \\
$2 \frac{1}{2} \times 11=2 \frac{7}{2} \times 12$ & is $=2 \frac{1}{2}$ times the length, \\
$2 \frac{1}{2} \times 12=2 \frac{1}{2}$ & for the contents, therefore \\
& $22 \mathrm{ft} . \times 2 \frac{1}{2}=55 \mathrm{ft}$. Ans.
\end{tabular}

Batten Shingle, No. 12.

\begin{tabular}{|c|c|c|c|c|c|c|c|}
\hline Lengths. 离高 & $\underset{\substack{x \\
-1 / 29}}{0}$ & $\underset{\substack{|c| \\
\text { Na }}}{x}$ & 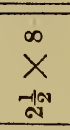 & $\begin{array}{c}\infty \\
x \\
-1 / N \\
\omega^{2}\end{array}$ & 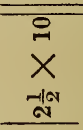 & 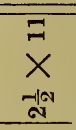 & 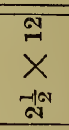 \\
\hline 20 & 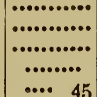 & $\ldots$ & $\begin{array}{r}\text {.......... } \\
8\end{array}$ & ${ }_{4}^{\cdots . .}$ & .. & $\ldots . . . .$. & $\ldots$ \\
\hline 21 & $\begin{array}{r}\cdots . . . . . . \\
8\end{array}$ & & 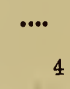 & ${ }_{4}$ & ${ }_{3}$ & ${ }_{4}^{\cdots . .}$ & ${ }_{3}$ \\
\hline 22 & & & ${ }_{3}$ & ${ }_{3}$ & ${ }^{*}{ }_{2}$ & $\cdot$ & $\begin{array}{r}\ldots . \\
\\
\end{array}$ \\
\hline 23 & $\ldots$ & & ${ }_{4}$ & ${ }_{4}$ & $\begin{array}{l}\cdots \\
\quad 3\end{array}$ & ${ }_{3}$ & ${ }_{4}$ \\
\hline 24 & $\ldots$ & $\begin{array}{r}. . . . . . . . \\
8\end{array}$ & ${ }_{4}$ & ${ }^{.}$ & $\cdot{ }_{1}$ & 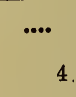 & $\ldots . . .$. \\
\hline 25 & $\begin{array}{r}\ldots . . . \cdot \\
6\end{array}$ & $\begin{array}{r}. . . \cdots \cdot . . . \cdot \\
9\end{array}$ & ${ }_{4}$ & ${ }_{3}$ & $\begin{array}{l}\cdots \\
\quad{ }_{3}\end{array}$ & ${ }_{3}$ & ${ }_{2}$ \\
\hline 26 & 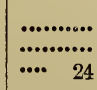 & $\quad+\cdots \cdot . .$. & ${ }^{\cdots . . .}$ & ${ }_{3}$ & ${ }^{\prime}{ }_{2}$ & $\cdot$ & ${ }_{3}$ \\
\hline
\end{tabular}


Rule for finding the Contents of Battens.

Divide the length of the piece by $4 \frac{4}{5}$, and multiply the product by the breadth of the piece, for the contents in feet; or multiply the length by the number given in the table for the contents.

Ans. 30 feet.

What are the contents of a batten 24 feet long $2 \frac{1}{2}$ by 6 ?

$2 \frac{1}{2} \times 6$, by the Table, is $=$ to $1 \frac{1}{4}$ times the length; $24 \times$ $1 \frac{1}{4}=30$ feet.

Second Solution. $-24 \div 4 \frac{4}{5}=5 ; 5 \times 6=30$ feet.

The specification is made out according to the last solution.

Specification of Batten Shingle, No. 12.

\begin{tabular}{|c|c|c|c|c|c|c|c|c|}
\hline Lengths. & 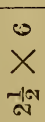 & $\begin{array}{c}\bar{x} \\
\text { - } \\
\text { and }\end{array}$ & $\begin{array}{c}\infty \\
\times \\
\underset{\sigma}{\infty}\end{array}$ & 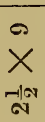 & $\begin{array}{c}\stackrel{0}{-1} \\
\times \\
\text { 워 }\end{array}$ & 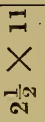 & $\begin{array}{c}\underset{N}{N} \\
\times \\
\text { Na }\end{array}$ & Contents. \\
\hline 20 & 45 & 15 & 8 & 4 & 2 & 12 & 4 & 2,812 \\
\hline 21 & 8 & 3 & 4 & 4 & 3 & 4 & 3 & 1,080 \\
\hline 22 & 9 & 4 & 3 & 3 & 2 & 1 & 3 & 917 \\
\hline 23 & 4 & 3 & 4 & 4 & 3 & 3 & 4 & 1,073 \\
\hline 24 & 12 & 8 & 4 & 2 & 1 & 4 & 12 & 1,880 \\
\hline 25 & 6 & 9 & 4 & 3 & 3 & 3 & 2 & 1,276 \\
\hline 26 & 24 & 8 & 4 & 3 & 2 & 1 & 3 & 1,765 \\
\hline & & & & & & & \multicolumn{2}{|c|}{ Total, 10,803} \\
\hline
\end{tabular}

Random Shingle No. 13, for any Dimension.

(Contents given in the Columns.)

\begin{tabular}{|c|c|c|c|c|c|c|c|c|c|c|c|c|c|c|}
\hline & $\stackrel{\infty}{x}$ & $\underset{\sim}{x}$ & $\stackrel{\infty}{x}$ & $\mid \begin{array}{l}a \\
x\end{array}$ & $\underset{\infty}{x}$ & $\underset{\infty}{\infty}$ & $\begin{array}{l}7 \\
x \\
x\end{array}$ & $\stackrel{\infty}{\times}$ & $\underset{x}{x}$ & $\mid \begin{array}{l}\stackrel{2}{x} \\
\stackrel{ }{O}\end{array}$ & 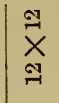 & $\begin{array}{l}\infty \\
\times\end{array}$ & & \\
\hline $\begin{array}{l}0 \\
0 \\
5 \\
5 \\
8\end{array}$ & $\begin{array}{l}10 \\
10\end{array}$ & $\begin{array}{l}210 \\
640 \\
120 \\
240 \\
150 \\
180 \\
200 \\
150\end{array}$ & $\begin{array}{c}150 \\
250 \\
350 \\
350 \\
350 \\
400 \\
500 \\
210 \\
240 \\
60\end{array}$ & $\begin{array}{l}120 \\
220 \\
110 \\
320 \\
150 \\
210 \\
641 \\
120\end{array}$ & $\begin{array}{l}120 \\
120 \\
100 \\
210 \\
1100 \\
250 \\
100 \\
100\end{array}$ & $\begin{array}{l}60 \\
60 \\
20 \\
30 \\
36 \\
12 \\
-\end{array}$ & $\begin{array}{l}250 \\
1 \overline{600} \\
5 \overline{500} \\
\overline{250} \\
-\end{array}$ & $\begin{array}{l}210 \\
420 \\
150 \\
600 \\
500 \\
120 \\
-\end{array}$ & \begin{tabular}{|l}
210 \\
410 \\
210 \\
312 \\
200 \\
100 \\
100 \\
-
\end{tabular} & $\begin{array}{l}120 \\
2500 \\
120 \\
120 \\
200 \\
120 \\
100 \\
-\end{array}$ & $\begin{array}{l}120 \\
120 \\
600 \\
150 \\
120 \\
2100 \\
100 \\
-\end{array}$ & $\left\{\begin{array}{l}160 \\
120 \\
150 \\
100 \\
200 \\
150 \\
200 \\
150 \\
150\end{array}\right.$ & $\left\{\begin{array}{l}200 \\
200 \\
200 \\
100 \\
100 \\
100 \\
100 \\
200 \\
-200\end{array}\right.$ & $\begin{array}{l}15 \\
12 \\
15 \\
10 \\
20 \\
12 \\
-\end{array}$ \\
\hline
\end{tabular}


Method of keeping Shingle No. 13.

The contents are found by the Board Rule and marked on each piece, and afterwards placed in the proper column in the shingle.

What is the total number of feet of merchantable spruce lumber in Random Shingle, No. 13.

Ans. 23,464 feet.

Random Shingle, No. 14.

(Running Lengths given in the Columns.)

\begin{tabular}{|c|c|c|c|c|c|c|c|c|c|c|c|}
\hline$\underset{\text { N }}{x}$ & $\underset{\infty}{\dot{x}}$ & $\underset{+}{x}$ & $\underset{*}{x}$ & $x_{10}^{10}$ & $\stackrel{\infty}{x}_{20}^{\infty}$ & $\underset{\omega}{x}$ & $\underset{1}{x}$ & ${ }_{1}^{\infty}$ & $\underset{\infty}{\stackrel{\overbrace{}}{x}}$ & $\underset{\sim}{\stackrel{N}{x}}$ & $\begin{array}{l}\text { Contents of the } \\
\text { whole. }\end{array}$ \\
\hline 100 & 80 & 100 & 200 & 72 & 120 & 100 & 120 & 20 & 120 & 50 & \multirow{13}{*}{$\begin{array}{r}3 \times 6=1,045 \\
2 \times 10 \equiv 2,833 \\
4 \times 8 \equiv 4,186 \\
4 \times 9 \equiv 2,970 \\
5 \times 5 \equiv 1,096 \\
5 \times 6 \equiv 2,137 \\
6 \times 6 \equiv 3,525 \\
7 \times 7 \equiv 2,797 \\
7 \times 9=1,118 \\
8 \times 10 \equiv 10,466 \\
10 \times 12=10,500\end{array}$} \\
\hline 100 & 60 & 150 & 100 & 72 & 100 & 100 & 100 & 18 & 150 & 20 & \\
\hline 25 & 40 & 210 & 75 & 60 & 150 & 120 & 100 & 16 & 120 & 40 & \\
\hline 125 & 20 & 110 & 60 & 40 & 100 & 200 & 40 & 24 & 100 & 20 & \\
\hline 100 & 15 & 200 & 40 & 18 & 110 & 110 & 20 & 20 & 100 & 100 & \\
\hline 200 & & & 20 & 19 & 70 & 120 & 100 & 18 & 200 & 100 & \\
\hline 100 & 28 & 150 & 10 & 20 & 60 & 150 & 6i) & 16 & 100 & 100 & \\
\hline 100 & 36 & 120 & 75 & 70 & 40 & 100 & 50 & 19 & 150 & - & \\
\hline 100 & 72 & 100 & 100 & 60 & 20 & 60 & 40 & 24 & 250 & 150 & \\
\hline 200 & 150 & 12 । & 150 & 40 & 30 & 40 & 20 & 20 & 100 & 200 & \\
\hline 150 & 96 & 150 & 60 & 20 & 20 & 20 & 15 & - & 120 & 120 & \\
\hline 400 & 100 & 160 & 100 & 15 & 20 & 25 & 20 & 18 & - & - & \\
\hline - & 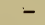 & . & - & 20 & 15 & 30 & - & 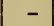 & 60 & 150 & \\
\hline 1700 & 697 & 1570 & 990 & 526 & 855 & 1175 & 685 & 213 & 1570 & 1050 & \multirow{5}{*}{ Total . $42,673 \mathrm{ft}$} \\
\hline & & 0 & & $? 1$ & $2 \frac{1}{12}$ & & $4 \frac{1}{12}$ & 51 & & & \\
\hline 1700 & 697 & 3140 & \multirow[t]{3}{*}{2970} & 1052 & 1710 & \multirow[t]{3}{*}{3525} & 2740 & 1065 & 9420 & \multirow[t]{3}{*}{10500} & \\
\hline 1133 & 348 & 1046 & & 44 & 427 & & 57 & 53 & 1046 & & \\
\hline 2833 & 1045 & 4186 & & 1096 & 2137 & & 2797 & 1118 & 10466 & & \\
\hline
\end{tabular}




\begin{tabular}{|c|c|}
\hline ¿ें & 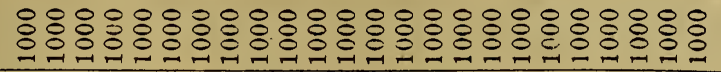 \\
\hline 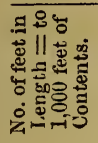 & 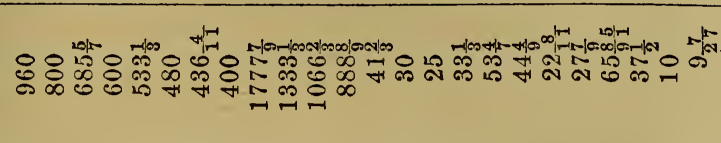 \\
\hline 离高官 & 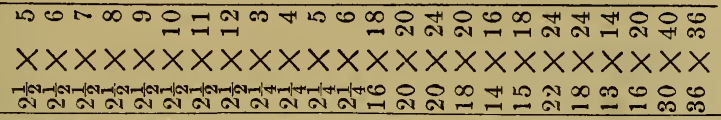 \\
\hline 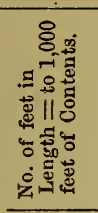 & 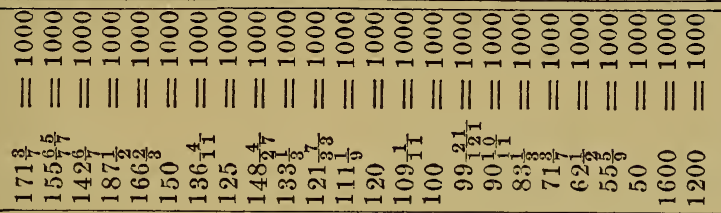 \\
\hline 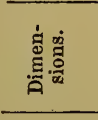 & 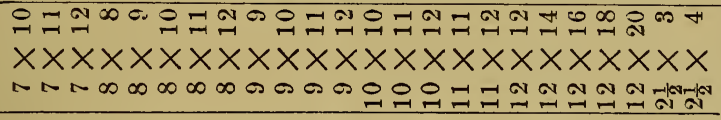 \\
\hline 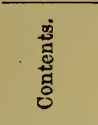 & 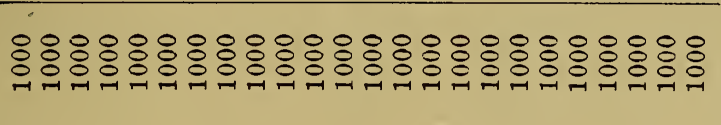 \\
\hline 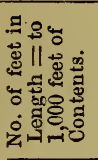 & 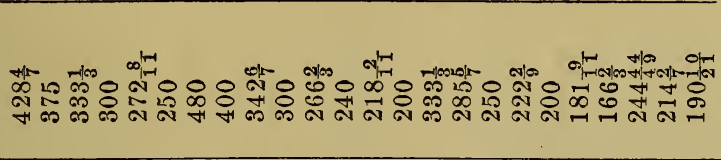 \\
\hline 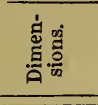 & 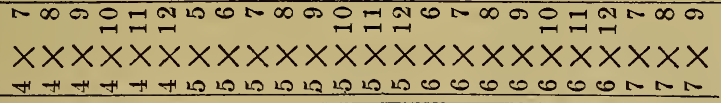 \\
\hline
\end{tabular}

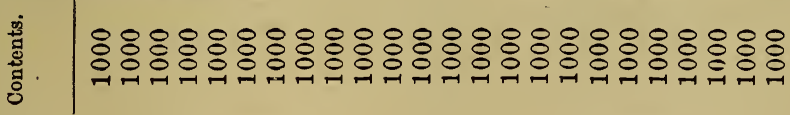

\begin{tabular}{|c|c|}
\hline 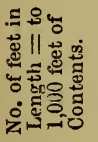 & 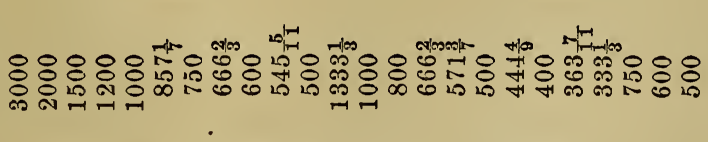 \\
\hline 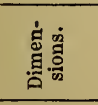 & 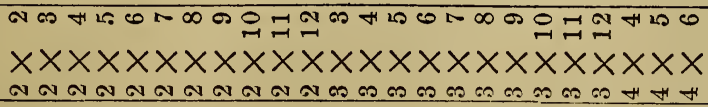 \\
\hline
\end{tabular}


Rule showing how Table $B$ is calculated.

Divide the area or contents of the end into the given number of feet of contents, and the quotient will be the number of feet of running lengths, equivalent to the given number of feet of contents.

1. What number of feet in length of 10 inches by 12 inches will be equal to 1,000 feet contents.

By the table 10 inches $\times 12$ inches is 10 times the length, for the contents ; therefore, $1,000 \div 10=100$ feet in length.

2. How many feet of $2 \times 3$ are equal to 1,000 feet of contents?

$2 \times 3=\frac{1}{2}$ the length ; therefore, $1,000 \times 2=2,000$ feet $=$ length required.

TABLE C.-Number of Feet of the following Dimensions of Timber that will make 1,000 Feet, Cubic or Solid Measurement.

\begin{tabular}{|c|c|c|c|c|c|}
\hline Dimensions. & $\begin{array}{l}\text { No. of Feet } \\
\text { in Length. }\end{array}$ & $\begin{array}{l}\text { Cubic } \\
\text { Feet. }\end{array}$ & Dimensions. & $\begin{array}{l}\text { No. of Feet } \\
\text { in Length. }\end{array}$ & $\begin{array}{l}\text { No. of ft. } \\
\text { of Cubic } \\
\text { Measure. }\end{array}$ \\
\hline $5 \times 5$ & 5,760 & 1,000 & $7 \times 12$ & $1,714 \frac{2}{7}$ & 1,000 \\
\hline $5 \times 6$ & 4,800 & 1,000 & $8 \times .8$ & 2,250 & 1,000 \\
\hline $5 \times 7$ & $4,114 \frac{2}{7}$ & 1,000 & $8 \times 9$ & 2,000 & 1,000 \\
\hline $5 \times$ & 3,600 & 1,000 & $8 \times 10$ & 1,800 & 1,000 \\
\hline $5 \times 9$ & 3,200 & 1,000 & $8 \times 11$ & $1,636_{\frac{1}{1} \mathrm{~T}}$ & 1,000 \\
\hline $5 \times 10$ & 2880 & 1,000 & $8 \times 12$ & 1,500 & 1,000 \\
\hline $5 \times 11$ & $2,618_{11}^{2}$ & 1,000 & $9 \times 9$ & $1,777 \frac{7}{9}$ & 1,000 \\
\hline $5 \times 12$ & 2,400 & 1,000 & $9 \times .10$ & 1,600 & 1,000 \\
\hline $6 \times 6$ & 4,000 & 1,000 & $9 \times 11$ & $1,455 \frac{5}{11}$ & 1,000 \\
\hline $6 \times 7$ & $3,428 \frac{4}{7}$ & 1,000 & $9 \times 12$ & $1,333 \frac{1}{3}$ & $1, \mathrm{CO0}$ \\
\hline $6 \times 8$ & 3,000 & 1,000 & $10 \times 10$ & 1,440 & 1,000 \\
\hline $6 \times 9$ & $2,666 \frac{2}{3}$ & 1,000 & $10 \times 11$ & $1,309_{1 T}^{1}$ & 1,000 \\
\hline $6 \times 10$ & 2,400 & 1,000 & $10 \times 12$ & 1,200 & 1,000 \\
\hline $6 \times 11$ & $2,181_{1 \mathrm{~T}}^{3}$ & 1,000 & $11 \times 11$ & $1,190 \frac{10}{121}$ & 1,000 \\
\hline $6 \times 12$ & 2,000 & 1,000 & $11 \times 12$ & $1,090 \frac{1}{1} \frac{0}{1}$ & 1,000 \\
\hline $7 \times 7$ & $2,938 \frac{3}{4} \frac{8}{9}$ & 1,000 & $12 \times 12$ & 1,000 & 1,000 \\
\hline $7 \times 8$ & $2,571 \frac{3}{7}$ & 1,000 & $14 \times 16$ & $642 \frac{6}{7}$ & 1,000 \\
\hline $7 \times 9$ & $2,285 \frac{5}{7}$ & 1,000 & $16 \times 18$ & 500 & 1,000 \\
\hline $7 \times 10$ & $2,057 \frac{1}{7}$ & 1,000 & $18 \times 20$ & 400 & 1,000 \\
\hline $7 \times 11$ & $1,870 \frac{1}{7} \frac{0}{7}$ & 1,000 & $20 \times 22$ & $327 \frac{3}{1 T}$ & 1,000 \\
\hline & - & 1,000 & $22 \times 24$ & 2728 & 1,000 \\
\hline
\end{tabular}


Rule showing how Table $C$ is computed.

Multiply the breadth and width in inches together, and divide the product by 144 , the number of inches in a square foot, and the quotient divided into the given number of cubic feet will give the number of feet in length, equal to said number of feet.

How many feet running length of 6 inches $\times 6$ inches are equal to 1,000 cubic feet?

Ans. 4,000 feet.

$6 \times 6=36 ; 36 \div 144=\frac{36}{144}=\frac{1}{4} ; \frac{1}{4}$ inverted $=$ to $\frac{4}{1}$ $\times \frac{1000}{1}=\frac{4000}{1}=4,000$ feet of running lengths $=1,000$ cubic feet.

Table showing the Numbers to multiply the Lengths of the following Dimensions by in order to find the Contents in Cubic Feet.

\begin{tabular}{|c|c|c|}
\hline Dimension. & Dimension. & Dimension. \\
\hline $5 \times 5=\frac{25}{144}$ & $7 \times 11=\frac{77}{4}$ & $12 \times 16=1 \frac{1}{3}$ \\
\hline $5 \times 6=\frac{5}{24}$ & $7 \times 12=\frac{7}{12}$ & $13 \times 14=$ \\
\hline $5 \times 7=\frac{35}{144}$ & $8 \times 8=\frac{4}{9}$ & $14 \times 16=$ \\
\hline $5 \times 8=\frac{5}{18}$ & $8 \times 9=\frac{1}{2}$ & $16 \times 18=$ \\
\hline $5 \times 9=\frac{5}{16}$ & $8 \times 10=\frac{5}{9}$ & $16 \times 20=$ \\
\hline $5 \times 10=\frac{2}{7} \frac{5}{2}$ & $8 \times 11=\frac{1}{1}$ & $18 \times 20=$ \\
\hline $5 \times 11=\frac{55}{144}$ & $8 \times 12=\frac{2}{3}$ & $20 \times 22=$ \\
\hline $5 \times 12=\frac{5}{12}$ & $9 \times 9=\frac{9}{16}$ & $22 \times 24=$ \\
\hline $6 \times 6=\frac{1}{4}$ & $9 \times 10=\frac{5}{8}$ & $24 \times 26=4 \frac{1}{3}$ \\
\hline $6 \times 7=\frac{7}{24}$ & $9 \times 11=\frac{1}{1} \frac{1}{6}$ & $26 \times 28=$ \\
\hline $6 \times 8=\frac{1}{3}$ & $9 \times 12=\frac{\overline{3}}{4}$ & $28 \times 30=$ \\
\hline $6 \times 9=\frac{3}{8}$ & $10 \times 10=\frac{2}{3} \frac{5}{6}$ & $30 \times 32=$ \\
\hline $6 \times 10=\frac{5}{12}$ & $10 \times 11=\frac{5}{7} \frac{5}{2}$ & $32 \times 34=7 \frac{5}{9}$ \\
\hline $6 \times 11=\frac{11}{24}$ & $10 \times 12=\frac{5}{6}$ & $34 \times 36=8 \frac{1}{2}$ \\
\hline $6 \times 12=\frac{1}{2}$ & $11 \times 11=\frac{121}{14}$ & $36 \times 38=9 \frac{1}{2}$ \\
\hline $7 \times \quad 7=\frac{49}{144}$ & $11 \times 12=\frac{1}{12}$ & $38 \times 40=10 \frac{5}{9}$ \\
\hline $7 \times 8=\frac{7}{18}$ & $12 \times 12=1$ & $40 \times 42=11 \frac{2}{3}$ \\
\hline $7 \times 9=\frac{7}{16}$ & $12 \times 14=1 \frac{1}{6}$ & $42 \times 44=12 \frac{5}{6}$ \\
\hline $7 \times 10=\frac{3}{7} \frac{5}{2}$ & & \\
\hline
\end{tabular}


QUESTIONS FOR EXERCISE.

1. Required the number of solid feet in a timber $B$ inches $\times 6$ inches and 40 feet long? Ans. 10 feet.

Solution. $-6 \times 6=\frac{1}{4}$ of length, therefore $\frac{1}{4}$ of $40=$ 10 feet.

2. What is the solidity of a piece of 6 -inch $\times 12$-inch timber 72 feet long?

Ans. 36 feet.

By the table $6 \times 12=\frac{1}{2}$ the length, for the contents; therefore $\frac{1}{2} \times 72=36$ feet.

3. What number of cubic feet are there in a piece of timber 40 feet long, 22 inches $\times 24$ inches?

Ans. $146 \frac{2}{3}$ feet.

4. Required the number of feet in a piece of timber 32 feet long, 5 inches $\times 12$ inches?

Solution. -32 feet $\times \frac{5}{1^{2}}=13 \frac{1}{3}$ feet $=$ contents.

5. What number of culic feet in the following pieces, namely, 6 pieces 60 feet long 12 inches $\times 16$ inches, and 12 pieces 35 feet long and 16 inches $\times 18$ inches?

Ans. 15,840 feet.

6. What are the contents in cubic feet of 6 pieces of 20 inches $\times 24$ inches and 35 feet long?

Ans. $111 \frac{2}{3}$ cubic feet.

7. What number of cubic feet in a piece of timber 28 inches $\times 30$ inches and 60 feet long? Ans. 350 cubic feet. Solution. $-60 \times 5 \frac{5}{6}=350$ feet of cubic measure.

8. Required the contents in cubic feet of a piece of pine timber 30 inches $\times 32$ inches and 30 feet in length?

Ans. 200 feet.

9. How many tons of timber (allowing 42 cubic feet to the ton) in a piece of timber 38 inches $\times 40$ inches and 45 feet long?

Ans. $11 \frac{1}{4}$ tons.

10. What will be the cost of a piece of pine timber 18 inches $\times 20$ inches and 30 feet in length @ 30 cents per cubic foot?

Ans. $\$ 22.50$. 
Rule to reduce Feet of Board Measure to Cubic Feet.

Divide the contents in superficial feet by 12 , and it will give the number of cubic feet; or multiply the number of cubic feet by 12 and the product will be feet of board measure.

In 1,200 feet of board measure how many cubic feet are there?

Ans. 100 cubic feet.

Solution. $-1,200 \div 12=100$ cubic feet.

Required the number of feet of board measure in 100 feet of cubic measure? Ans. 1,200 feet.

$100 \times 12=1,200$ feet of board measure.

Second Method of making out a Specification.

3-INCH SPECIFICATION BY THE SECOND METHOD.

\begin{tabular}{|c|c|c|c|c|c|c|c|c|}
\hline Lengths. & $\underset{\infty}{\dot{X}}$ & $\hat{x}$ & $\begin{array}{l}\infty \\
\times \\
\infty\end{array}$ & $\underset{\infty}{\infty}$ & $\mid \begin{array}{l}\stackrel{\rho}{e} \\
x \\
\infty\end{array}$ & $\underset{\approx}{Z}$ & $\begin{array}{c}N \\
X \\
\infty\end{array}$ & Contents. \\
\hline 14 & 2 & 3 & 4 & 6 & 8 & 4 & 6 & \\
\hline 15 & 4 & 2 & 1 & 4 & 2 & 8 & 4 & \\
\hline 16 & 2 & 4 & 2 & 1 & 3 & 2 & 4 & \\
\hline 17 & 6 & & 1 & & 1 & 3 & 2 & \\
\hline 18 & 8 & 4 & 6 & 1 & 3 & 2 & 4 & \\
\hline 19 & 2 & 1 & 2 & 3 & 2 & 4 & 6 & \\
\hline 20 & 3 & 2 & 1 & 4 & 2 & 1 & 3 & \\
\hline 21 & 6 & 4 & 8 & 2 & 1 & 3 & 2 & \\
\hline 22 & 1 & 5 & 4 & 3 & 2 & 1 & 1 & \\
\hline 23 & 2 & 1 & 10 & 4 & 1 & 2 & 1 & \\
\hline 24 & & 6 & & 4 & & 3 & 2 & \\
\hline 25 & 4 & & 2 & 8 & 6 & & 4 & \\
\hline 26 & & 3 & 2 & 1 & & 2 & 8 & \\
\hline 27 & 6 & 5 & 1 & & 3 & & 2 & \\
\hline 28 & & 8 & & 2 & & 4 & 6 & \\
\hline 29 & 3 & & 5 & 2 & 1 & 6 & 4 & \\
\hline & 0 & 364 & & 140 & 1717 & $2563 \mid$ & 3807 & 15,693 feet \\
\hline
\end{tabular}




\section{Second Rule for Specifications.}

Multiply the number of pieces or dots in each square of the specification by the length of one of the pieces; and multiply the product thus found by $\frac{1}{4}$ of the breadth of said pieces for the contents in board measure of 3 -inch deals; by $\frac{1}{3}$ of the breadth for 4-inch; by $\frac{1}{6}$ of it for plank, etc.

Example showing how to make out the Three-inch Specifcation by Second Method.

\begin{tabular}{|c|c|}
\hline First Column 6 inches wide. & Second Column 7 inches wide. \\
\hline $\begin{array}{l}14 \times 2=28 \\
15 \times 4=60 \\
16 \times 2=32 \\
17 \times 6=102 \\
18 \times 8=144 \\
19 \times 2=38 \\
20 \times 3=60 \\
21 \times 6=126 \\
22 \times 1=22 \\
23 \times 2=46 \\
25 \times 4=100 \\
27 \times 6=162 \\
29 \times 3=87\end{array}$ & $\begin{array}{l}14 \times 3=42 \\
15 \times 2=30 \\
16 \times 4=64 \\
18 \times 4=72 \\
19 \times 1=19 \\
20 \times 2=40 \\
21 \times 4=84 \\
22 \times 5=110 \\
23 \times 1=23 \\
24 \times 6=144 \\
26 \times 3=78 \\
27 \times 5=135 \\
28 \times 8=224\end{array}$ \\
\hline $\begin{array}{l}\frac{1,007}{1,007} \\
\text { Contents, } 1,510 \text { feet. } \\
6 \text { inches, the breadth, di- } \\
\text { vided by } 4 \text { is }=\text { to } 1 \frac{1}{2}, \text { and } \\
1 \frac{1}{2} \times 1,007=1,510 \text {, the } \\
\text { contents. }\end{array}$ & $\begin{array}{l}\frac{1,065}{1,065} \\
\text { Contents, } 1,864 \text { feet. } \\
7 \text { inches, the breadth, di- } \\
\text { vided by } 4 \text { is }=\text { to } 1 \frac{3}{4} \text {, and } \\
1 \frac{3}{4} \times 1,065=1,864 \text { feet }= \\
\text { contents. }\end{array}$ \\
\hline
\end{tabular}

English deal specifications are generally made out by the second method. Both rules will give the same results. 
ON LUMBER SURVEYING.

Specification of Philadelphia Deal Shingle.

\begin{tabular}{|c|c|c|c|c|c|}
\hline Lengths. & $\begin{array}{l}\underset{\sim}{\Psi} \\
\underset{\infty}{X}\end{array}$ & Contents. & Lengths. & $\begin{array}{l}\underset{y}{X} \\
\times\end{array}$ & Contents. \\
\hline 14 & 40 & 1,680 & 28 & 14 & 1,176 \\
\hline 16 & 35 & 1,680 & 30 & 8 & 720 \\
\hline 18 & 30 & 1,620 & 32 & 4 & 384 \\
\hline 20 & 11 & 660 & 34 & 4 & 408 \\
\hline 22 & 9 & 594 & 36 & 7 & 756 \\
\hline 24 & 21 & 1,512 & 38 & 14 & 1,596 \\
\hline 26 & 6 & 468 & 40 & 14 & 1,680 \\
\hline \multicolumn{6}{|c|}{ Contents, } \\
\hline
\end{tabular}

Philadelphia Deal Shingle.

\begin{tabular}{|c|c|c|c|}
\hline 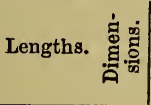 & $\underset{\infty}{\stackrel{N}{X}}$ & Lengths. & $\underset{\infty}{\stackrel{\sim}{x}}$ \\
\hline 14 & 40 & 28 & $\begin{array}{r}\text {............... } \\
14 \\
\end{array}$ \\
\hline 16 & $\begin{array}{r}35 \\
\end{array}$ & 30 & 8 \\
\hline 18 & $\begin{array}{r}30 \\
\end{array}$ & 32 & $\cdots$ \\
\hline 20 & $\begin{array}{r}\ldots . . . . . . . . \bullet \\
11 \\
\end{array}$ & 34 & $\begin{array}{r}\cdots . . \\
\quad 4 \\
\end{array}$ \\
\hline 22 & $\begin{array}{r}\cdots \cdots \cdots . . . . \\
9 \\
\end{array}$ & 36 & $\begin{array}{l}\cdots . . . . . \\
\\
\end{array}$ \\
\hline 24 & $\begin{array}{r}\ldots . . . . . \cdots . . . \\
21 \\
\end{array}$ & 38 & 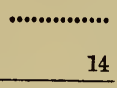 \\
\hline 26 & \begin{tabular}{cc|}
$\cdots \cdots . .$. & \\
& 6 \\
\end{tabular} & 40 & $\begin{array}{r}0 . \cdots . . . . . . \\
14 \\
\end{array}$ \\
\hline
\end{tabular}


The specification of Philadelphia deals is done the same as the 3-inch specification; or multiply the running lengths by 3 for the contents in feet of board measure. Philadelphia deal is generally 12 inches wide and even lengths, from 14 feet up, and the best quality of spruce lumber. English deals generally comprise all deals too short, or not good enough for Philadelphia or New York deals. Also short timber, battens, and plank, not suitable for other markets, go into the English deal pile. Deals that are knotty, cracked by the sun, or stained, or having wanes on them, and not poor enough for refuse, go to the English deal pile. New York deal must be the best quality of spruce, from 14 feet long up.

\section{Directions showing how to measure all kinds of Lumber by the Board Rule.}

Lay your rule across the board to be measured, at right angles to the further edge of the board, and let the outside edge of the board and further end of the rule be both even on that side, then observe the length of your board and turn your rule to the same length, then look on the line or column of that length, and you will find the contents marked on the rule just over the inside edge of the board.

\section{EXAMPLES FOR PRACTICE.}

1. What are the contents of a $1 \frac{1}{4}$-inch board 16 feet long and 12 inches wide?

Ans. 20 feet.

By the rule the contents given for 1-inch board is 16 feet contents, to which add $\frac{1}{4}$ of the contents, which will give the contents for $1 \frac{1}{4}$-inch boards. $16 \div 4=4 ; 16+4=$ 20 feet contents.

2. What are the contents of a board 32 feet long and 12 inches wide?

Ans. 32 feet.

As there is no 32 on my rule, I find the contents by the rule of a board, half the length to be 16 feet; which being doubled, gives the contents required $=32$ feet.

3. What are the contents of a $1 \frac{1}{2}$-inch board 20 feet long and 12 inches wide?

Ans. 30 feet. 
By the rule an inch board 20 feet long and 12 inches wide will contain 20 feet, to which add half of 20 for the contents of a $1 \frac{1}{2}$-inch board. $20 \div 2=10 ; 20+10=30$ feet.

4. Required the contents of a plank 24 feet long 2 inches $\times 12$ inches?

Ans. 48 feet.

By the board rule, in a board 24 feet long 12 inches wide and 1 inch thick there are 24 feet, and as plank is 2 inches thick, therefore twice the contents of the face of it will be equal to the true contents, $24 \times 2=48$ feet.

\section{Rule for any Dimension.}

Multiply the number of feet in the face of the piece to be measured, by the thickness in inches, and it will give the contents in feet of board measure.

Rule for measuring Logs or Round Timber.

Multiply the length, taken in feet, by the square of one fourth of the mean girth, taken in inches, and this product divided by 144 will give the contents in cubic feet.

Note. - The girth of tapering timber is usually taken about one third the distance from the larger to the smaller end. The rule is that in common use, though very far from giving the actual number of cubic feet; 40 cubic feet as given by the rule are in fact $=50 \frac{92}{100}$ true cubic feet.

\section{EXAMPLE.}

1. How many cubic feet in a stick of timber which is 40 feet long, and whose girth is 60 inches? Ans. $62 \frac{1}{2}$ feet.

$60 \div 4=15$ inches $=\frac{1}{4}$ of girth $; 15 \times 15=225=$ square of quarter of the girth; $225 \times 40$ feet $=9,000$; $9,000 \div 144=62 \frac{1}{2}$ cubic feet.

2. How many cubic feet in a piece of timber 21 feet long, and whose girth is 36 inches?

3. What are the contents of a $\log 100$ feet long, and whose girth is 150 inches? 
To find the largest Square Piece of Timber that may be sawed from a Round Stick of Timber, having the Diameter or Circumference of the Snall End given.

Rule 1. - Multiply the given diameter by .707106 , or,

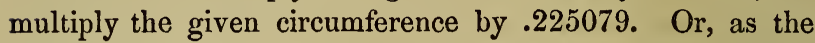
diameter of a circle is equal to the diagonal of the inscribed square -

Rule 2. - Square the diameter and take half the sum of the square, and extract the square root of it,

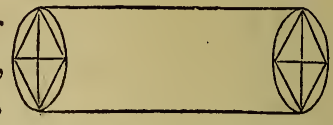
a.cl the root thus found will be the side of the inscribed square.

\section{EXAMPLE.}

1. I have a piece of timber 30 inches in diameter; how large a square stick can be hewn from it.

By the last rule 30 squared $=30 \times 30=900 ; 900 \div 2$ $=450 ; \sqrt{4}^{\frac{2}{50}}=21.21+$ inches square.

2. How large a square stick may be hewn from a piece of round timber 120 inches in circumference?

3. How large a square stick may be sawn from a piece of round timber 60 inches in diameter?

Having the Side of a Square Stick given, to find the Diameter of the Tree from which it was sawn.

Rule. - Square the side and double it, and out of the product extract the square root.

What must be the diameter of a tree that when hewn shall be 18 inches square?

Ans. 25.44 inches.

\section{TABLE.}

12 lines $=1$ inch.

12 inches $=1$ foot.

3 feet $=1$ yard.

Inches multiplied by inches produce

Parts marked thus '.

Parts by parts give fourths, marked thus ' $'$. 
Inches are marked '.

144 square inches make 1 square foot.

9 square feet $=1$ square yard.

1,728 cubic inches $=1$ cubic foot.

50 cubic feet $=1$ load.

40 cubic feet $=1$ ton of timber.

16 cubic feet $=1$ cord foot.

8 cord feet, or 128 cubic feet $=1$ cord of wood.

1,980 feet superficial $=1$ St. Petersburg standard of deals.

Form of a Bill of Lading of Timber, Shingle No. 8, etc., etc.

SHIPPED, in good order and condition, by Edmond B. Sanderson \& Co., on board the good ship "Southern," whereof James Brown is master for this present voyage, now lying in the port of New York, U. S., and bound for Liverpool, England. To say :-

47,928 ft. Mer. spruce, all under deck, $100 \mathrm{M}$ spruce laths, all under deck, $80 \mathrm{MI} \mathrm{ft}$. Mer. pine, all on deck,

being marked and numbered as in the margin; and are to be delivered, in like good order and condition, at the aforesaid port of Liverpool (the danger of the seas and fire always excepted), unto David Belt \& Sons, or to assigns, he or they paying freight for the said timber at the rate of ten dollars per $\mathbf{M}$ feet, and one dollar per $\mathbf{M}$ for laths, without primage and average accustomed.

In witness whereof, the master of the said vessel hath affirmed to three bills of lading, all of this tenor and date; one of which being accomplished, the others to stand void.

Dated at NEw YoRK, U.S.,

JAMES BROWN.

May the $3 d$, A. D. 1870. 


\title{
Bill of Lading.
}

\begin{abstract}
ShIPped, in good order and condition, by $\mathrm{T}$. Pandol \& Co., on board the good schooner called the "Northern Dawn," whereof Daniel E. Bloomer is master for this present voyage, now lying in the port of Bangor, Me., and bound for New York. To say:-

$110 \mathrm{M}$ feet hemlock lumber, all under deck,

75 M feet spruce lumber, all on deck,

120 M laths, all on deck,

being marked and numbered as in the margin; and are to be delivered, in like good order and condition, at the aforesaid port of New York (the danger of the seas and fire only excepted), unto Messrs. Denton and Beeters, or to assigns, he or they paying freight for the said lumber at the rate of four dollars per $\mathbf{M}$ feet, and sixty cents per $\mathbf{M}$ for laths, without primage and average accustomed.

In witness whereof, the master of the said vessel hath affirmed to three bills of lading, all of this tenor and date; one of which being accomplished, the others to stand void.

Daniel E. Bloomer.

Dated at BAngor, ME.,

June the $3 d, 1869$.
\end{abstract}

Surveyor's Bill for Services rendered.

BANgor, ME., June the 2d, 1869.

Messrs. Dunton \& Boomer,

To Daniel E. Shaw, surveyor, Dr.

For surveying $250 \mathrm{M} \mathrm{ft}$. of spruce lumber to

schooner “Juno," @ 25c. per M . . . . . . \$62.50 
Survey Bill of Lumber, etc.

Surveyed from James E. Dale \& Sons, of Clinton, Iowa, to schooner "Pallas," Captain Dunn. To say:-

$36,500 \mathrm{ft} .2 \times 6$, from $12 \mathrm{ft}$. long up (mch.), spruce.

35,600 "No. 1 pine boards.

22,400 " hemlock boards (mch.).

15,000 " $8 \times 10$ Mer. pine timber.

250 M No. 1 pine shingles.

Thomas B. Proddfoor,

Clinton, Iowa,

Surveyor.

June the 12th, Anno Domini 1869.

\section{Surveyor's Receipt.}

$\$ 62.50$.

Bangor, Me., June the 4th, A.D. 1869.

Received from Messrs. Dunton \& Boomer sixty-two dollars and fifty cents, which pays for surveying 250 M feet of spruce lumber to schooner "Juno," @ 25̃c. per M.

Daniel E. Shaw, Surveyor.

\section{NOVEL RULES}

For finding the Contents of Plank, Deal, Battens, Joist, and Timber, by multiplying a Fractional Part of the Length by the Breadth.

2-inch is $\frac{1}{6}$ of the length multiplied by the breadth, for the contents.

3 -inch is $\frac{1}{4}$ of the length multiplied by the breadth, for the contents.

4-inch is $\frac{1}{3}$ of the length multiplied by the breadth, for the contents.

5 -inch is the length divided by $2 \frac{2}{5}$, and the quotient multiplied by the breadth. 
6-inch is $\frac{1}{2}$ of the length multiplied by the breadth, for the contents.

7 -inch is the length divided by $1 \frac{5}{7}$, and the quotient multiplied by the breadth.

8-inch is the length divided by $1 \frac{1}{2}$, and the quotient multiplied by the breadth.

9-inch is the length divided by $1 \frac{1}{3}$, and the quotient multiplied by the breadth.

10-inch is the length divided by $1 \frac{1}{5}$, and the quotient multiplied by the breadth.

11-inch is the length divided by $1 \frac{1}{1}$, and the quotient multiplied by the breadth.

12-inch, multiply the length by the width, for the contents.

$2 \frac{1}{2}$-inch, or battens, is the length divided by $4 \frac{4}{5}$, and the quotient multiplied by the breadth.

P. S. - The above rules give the contents in feet of board measure.

\section{EXAMPLES FOR PRACTICE.}

1. Required the contents in superficial feet of a piece of timber 10 inches $\times 12$ inches and 40 feet long.

Ans. 400 feet.

Solution. - By the table, 10 inches is $1 \frac{1}{5}$ of the length multiplied by the breadth. Therefore 40 feet $\div 1 \frac{1}{5}=\frac{40}{1} \times$ $\frac{5}{6}=2 \frac{00}{6} 0=33 \frac{1}{3} ; 33 \frac{1}{3} \times 12=400$ feet.

2. What are the contents of a piece of timber 12 inches $\times 20$ inches, and 40 feet long? Ans. 800 feet. Solution. $-40 \times 20=800$ feet.

3. What are the contents of a plank 2 inches $\times 11$ inches and 36 feet long?

Ans. 66 feet.

Solution. -2 inches is $\frac{1}{6}$ of the length. Therefore $36 \div 6=6 ; 6 \times 11=66$ feet.

4. What are the contents of a piece of timber 8 inches $X$ 11 inches and 40 feet in length? Ans. $293 \frac{1}{3}$ feet.

Şolution. $-40 \div 1 \frac{1}{2}=\frac{40}{1} ; \frac{40}{1} \times \frac{2}{3}=\frac{80}{3}=26 \frac{2}{3} ; 26 \frac{2}{3}$ $\times 11=293 \frac{1}{3}$ feet. 
Given the Breadth of a Rectangular Plank in Inches, to find how much in Length will make a Foot, or any other required Quantity.

Rule. - Divide 144, or the area to be cut off, by the breadth in inches, and the quotient will be the length in inches.

1. If a board be 6 inches broad, what length of it will make a square foot? Ans. 2 feet. Solution. -144 inches $\div 6$ inches $=24$ inches; 24 inches $\div 12$ inches $=2$ feet.

2. If a plank be 2 inches $\times 8$ inches in size, what length of it will make 4 square feet?

Ans. 3 feet.

Solution. $-2 \times 8=16$, area of the end ; $144 \div 16=9$ inches for 1 foot, which, being multiplied by $4=4 \times 9=$ 36 inches $=3$ feet.

To find the Solid Contents of a Piece of Timber tapering regularly.

Rule. - Multiply the sum of the breadths of the two ends by the sum of the depths, to which add the product of the breadth and depth of each end; $\frac{1}{6}$ of this sum, multiplied by the length, will give the exact solidity of any piece of squared timber tapering regularly.

1. How many feet in a piece of manogany whose ends are rectangles, the length and breadth of one being 14 and 12 inches, and the corresponding dimensions of the other end 6 and 4 inches; also the length $30 \frac{1}{2}$ feet?

Solution. Ans. $18 \frac{2}{27}$ cubic feet.

$$
\begin{aligned}
& 14+6=20 \\
& 12+4=16
\end{aligned}
$$$$
12 \times 14=168
$$$$
6 \times 4=24
$$$$
20 \times 16=320
$$

512 sq. in. $=\frac{32}{9}$ sq. ft.

Then $\frac{1}{6} \times \frac{32}{9} \times 30 \frac{1}{2}=18 \frac{2}{27}$ cubic feet. 
When a Board or Plank is broader at one End than the other, to find what Length of it will make a Foot, or any other required Quantity.

Rule. - To the square of the product of the length and narrow end add twice the continual product of these quantities; namely, the length, the difference between the breadths of the ends, and the area of the part required to be cut off. Extract the square root of the sum; from the result deduct the product of the length and narrow end, and divide the remainder by the difference between the breadths of the ends.

\section{EXAMPLE.}

It is required to cut off 60 inches from the smaller end of a board; $\mathrm{A} D$ being 3 inches, $\mathrm{C} \mathrm{E} 6$ inches, and A B. 20 inches.

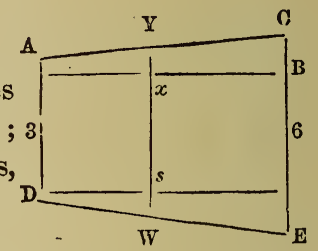

Here $\mathrm{A} x=\frac{1}{2 \mathrm{BC}}\left(\vee\left\{(\mathrm{A} \mathrm{B} \times \mathrm{AD})^{2}+4 \mathrm{~B} \mathrm{C} \times\right.\right.$ $\mathrm{A} \mathrm{B} \times 60\}-\mathrm{A} \mathrm{B} \times \mathrm{A} \mathrm{D}=\frac{1}{3}\left(\vee\left\{(20 \times 3)^{2}+\right.\right.$ $6 \times 20 \times 60\}-20 \times 3=14.64$, the length required.

To find how much in Length will make a Solid Foot, or any other required Quantity, of Squared Timber, of equal Dimensions from End to End.

Rule. - Divide 1,728 - the solid inches in a foot, or the solidity to be cut off - by the area of the end in inches.

1. If a piece of timber be 14 inches broad and 10 inches deep, how much of it will make a solid foot?

Ans. $12 \frac{12}{3} \frac{2}{5}$ inches, the length required.

$10 \times 14=140 ; 1,728 \div 140=12 \frac{12}{3}$ inches. 
Rule. - Multiply the area corresponding to the quarter girt in inches, by the length of the piece in feet, and the product will be the solidity. If the quarter girt exceeds the limits of the table, take $\frac{1}{2}$ of it, and 4 times the contents thus found will give the required contents.

A Table for Measuring Timber.

\begin{tabular}{|c|c|c|c|c|c|}
\hline Quarter Girt. & Area. & Quarter Girt. & Area. & Quarter Girt. & Area. \\
\hline $\begin{array}{c}\text { Inches. } \\
6\end{array}$ & $\begin{array}{l}\text { Feet. } \\
.250\end{array}$ & Inches. & Feet. & Inches. & \\
\hline $\begin{array}{l}6 \\
6 \frac{1}{4}\end{array}$ & .250 .272 & $\begin{array}{l}12 \\
121\end{array}$ & 1.000 & 18 & 2.250 \\
\hline $\begin{array}{l}0 \frac{4}{4} \\
6 \frac{1}{9}\end{array}$ & .294 & $\begin{array}{l}12 \frac{1}{4} \\
121 .\end{array}$ & 1.042 & $18 \frac{1}{2}$ & 2.376 \\
\hline & & $12 \frac{1}{2}$ & 1.085 & 19 & 2.506 \\
\hline $6 \frac{3}{4}$ & & $12 \frac{3}{4}$ & 1.129 & $19 \frac{1}{2}$ & 2.640 \\
\hline 7 & .340 & 13 & 1.174 & 20 & 2.777 \\
\hline $7 \frac{1}{4}$ & .364 & $13 \frac{1}{4}$ & 1.219 & $20 \frac{1}{2}$ & 2.917 \\
\hline $7 \frac{1}{2}$ & .390 & $13 \frac{1}{2}$ & 1.265 & 21 & $\begin{array}{l}3.062 \\
\end{array}$ \\
\hline $7 \frac{3}{4}$ & .417 & $13 \frac{3}{4}$ & 1.313 & $21 \frac{1}{2}$ & $\begin{array}{l}3.209 \\
\end{array}$ \\
\hline 8 & .444 & 14 & 1.361 & 22 & \begin{tabular}{|l|}
3.362 \\
\end{tabular} \\
\hline $8 \frac{1}{4}$ & .472 & $14 \frac{1}{4}$ & 1.410 & $22 \frac{1}{2}$ & 3.516 \\
\hline $8 \frac{1}{2}$ & .501 & $14 \frac{1}{2}$ & 1.460 & 23 & \begin{tabular}{|l|}
3.673 \\
\end{tabular} \\
\hline $8 \frac{3}{4}$ & .531 & $14 \frac{3}{4}$ & 1.511 & $23 \frac{1}{2}$ & 3.835 \\
\hline 9 & .562 & 15 & 1.562 & 24 & 4.000 \\
\hline $9 \frac{1}{4}$ & .594 & 154 & 1.615 & $24 \frac{1}{2}$ & 4.168 \\
\hline $9 \frac{1}{2}$ & .626 & $15 \frac{1}{2}$ & 1.668 & 25 & 4.340 \\
\hline $9 \frac{3}{4}$ & .659 & $15 \frac{3}{4}$ & 1.722 & $25 \frac{1}{2}$ & 4.516 \\
\hline 10 & .694 & 16 & 1.777 & 26 & 4.694 \\
\hline $10 \frac{1}{4}$ & .730 & $16 \frac{1}{4}$ & 1.833 & $26 \frac{1}{2}$ & 4.876 \\
\hline $10 \frac{1}{2}$ & .766 & $16 \frac{1}{2}$ & 1.890 & 27 & 5.062 \\
\hline $10 \frac{3}{4}$ & .803 & $16 \frac{3}{4}$ & 1.948 & $27 \frac{1}{2}$ & 5.252 \\
\hline $111^{2}$ & .840 & $17^{\circ}$ & 2.006 & 28 & 5.444 \\
\hline $11 \frac{1}{4}$ & .878 & $17 \frac{1}{4}$ & 2.066 & $28 \frac{1}{2}$ & 5.640 \\
\hline $11 \frac{1}{2}$ & .918 & $17 \frac{1}{2}$ & 2.126 & 29 & 5.840 \\
\hline $11 \frac{3}{4}$ & .959 & $17 \frac{3}{4}$ & 2.187 & $29 \frac{1}{2}$ & 6.044 \\
\hline
\end{tabular}

1. Required the contents of a piece of timber whose length is 30 feet and quarter girt is $17 \frac{3}{4}$ inches.

Ans. 65.610 feet. 
Solution by the Table. - Look for the quarter girt 17 $\frac{3}{4}$, in the column marked Quarter Girt, and in the adjoining column marked Area, will be found 2.186, which multiplied by the length, 30 feet, will be 65.610 feet for the solid contents.

Table showing the Weight in Pounds and Decimals of a Pound Avoirdupois of one Cubic Foot of the following Kinds of Wood.

\begin{tabular}{|c|c|c|c|}
\hline Cork Wood & 15.00 & Maple and Riga Fir & 46.87 \\
\hline Poplar & 23.94 & Ash and Dantzic Oak. & 47.50 \\
\hline Larch or Hackmatack & 34.00 & Apple Tree & 49.56 \\
\hline Elm and West India Fir & 34.75 & Alder & 50.00 \\
\hline Mahogany . & 35.00 & Oak, Canadian & 54.50 \\
\hline Pitch Pine. & 41.25 & Boxwood, French & 57.00 \\
\hline Cedar & 37.25 & Logwood & 57.06 \\
\hline Pear Tree. & 41.31 & Oak, English . & 51.87 \\
\hline Walnut. & 41.94 & Oak, sixty years old & 73.12 \\
\hline Elder Tree & 43.44 & Ebony & 83.18 \\
\hline Beech . . & 43.50 & Lignum Vitæ . & 83.31 \\
\hline Cherry Tree & 44.68 & & \\
\hline
\end{tabular}

\section{Rule for finding the Weight of any kind of Timber.}

Multiply the number of cubic feet it contains by the weight of one cubic foot of said timber.

\section{EXAMPLES.}

1. What is the weight of a piece of hackmatack timber 8 inches $\times 12$ inches, and 30 feet long?

By the table given of cubic measure, 8 inches $\times 12$ inches is $\frac{2}{3}$ of the length, for the contents : therefore $30 \div \frac{2}{3}$ $=20$ feet, contents.

By the table of weights a cubic foot of hackmatack is $=$ to 34 lbs., therefore $34 \times 30=1,020$ lbs. avoirdupois. 
2. What is the weight of a piece of Canadian oak 12 inches $\times 12$ inches, and 30 feet long? Ans. 1,635.00 lbs.

3. What is the weight of a piece of French boxwood 10 inches $\times 12$ inches, and 24 feet in length?

By the table of cubic measure, 10 inches $\times 12$ inches is $\frac{5}{6}$ of the length, for the contents in cubic feet; therefore 24 $\div \frac{5}{6}=20$ feet, contents ; $20 \times 57=1,140$ lbs. = weight required.

P. S. - The weight of any substance may be found as above, by finding the weight of 1 cubic foot and multiplying said weight by the contents.

\section{TONNAGE OF VESSELS.}

\section{Government Rule. English.}

For vessels aground, the length is to be measured on a straight line àlong the rabbet of the keel, from a perpendicular, let fall from the back of the main-post, at the height of the wing-transom, to a perpendicular at the height of the upper deck (but the middle deck of three-decked ships), from the forepart of the stern; then from the length between these perpendiculars subtract three fifths of the extreme breadth for the rake of the stern; and $2 \frac{1}{2}$ inches for every foot of the height of the wing-transom above the lower part of the rabbet of the keel, for the rake abaft; and the remainder will be the length of the keel for tonnage. The main breadth is to be taken from the outside of the outside plank, in the broadest part of the ship either above or below the wales, deducting therefrom all that it exceeds the thickness of the plank of the bottom, which shall be accounted the main breadth; so that the moulding breadth, or the breadth of the frame, will then be less than the main breadth, so found, by double the thickness of the plank of the bottom.

Rule. - Then multiply the length of the keel for tonnage, by the main breadth, so taken, and the product by half the 
breadth ; then divide the whole by 94 , and the quotient will be the tonnage.

In cutters and brigs, where the rake of the stern-post exceeds $2 \frac{1}{2}$ inches to every foot in height, the actual rake is generally subtracted instead of the $2 \frac{1}{2}$ inches to every foot, as before mentioned.

1. Suppose the length from the fore-part of the stern, at the height of the upper deck, to the after-part of the sternpost, at the height of the wing-transom to be 115 feet 8 inches, the breadth from outside to outside 40 feet 6 inches, and the height of the wing-transom 21 feet 10 inches, what is the tonnage?

ft. in.

406 breadth

$\frac{3}{403} \times 3=120.9 ; 120.9 \div 5=24.15$.

21.10 height of wing-transom $21.10 \times 2 \frac{1}{2}=54 \frac{7}{12} ; 54_{\frac{1}{2}}^{\frac{7}{2}}$ $\div 12=4.55 ; 4.55+24.15=28.70 ; 155.66-28.70=$ 126.96 = length.

$\frac{126.96 \times 40.25 \times 20.125}{94}=1,094$, the tonnage required.

2. If the length of the keel be 120 feet, and the breadth 40 feet, what is the tonnage? Ans. $1,021 \frac{13}{4}$ tons.

Solution. $-120 \times 40=4,800 ; 4,800 \times 20=96,000$; $96,000 \div 94=1,021 \frac{1}{4} \frac{3}{7}$ tons.

3 . If the length of the keel be 80 feet, and the breadth of the beam 36 feet, what is the tonnage? Ans. 551 $\frac{23}{4}$.

4. If the length of the keel be 460 feet, and the breadth of the beam 80 feet, what is the tonnage.

Ans. 15,659 tons.

Some divide the last product by 100 , to find the tonnage of king's ships, and by 95 , to find that of merchant ships.

\section{American Government Rule.}

For single-decked vessels. - Take the length on deck from the forward side of the main stern to the after-side of the stern-post, and the breadth at the broadest part above the 
main wales; take the depth from the under side of the deck plank to the ceiling of the hold, and deduct from the length three fifths of the breadth; multiply the remainder by the breadth, and the product by the depth, and divide the last product by 95 .

For double-decked vessels. - Proceed as with single-decked vessels, except for the depth take half the breadth.

\section{GAUGING.}

Gauging signifies the art of measuring all kinds of vessels and determining their capacity or the quantity of fluid or other matter they contain. It is usual to divide casks into four varieties, which are judged of from the greater or less apparent curvature of their sides, namely :-

1. The middle frustum of a spheroid.

2. The middle frustum of a parabolic spindle.

3. The two equal frustums of a paraboloid.

4. The two equal frustums of a cone.

282 cubic inches make 1 ale gallon, or beer.

231 cubic inches make 1 wine gallon.

21,504 cubic inches make 1 malt bushel.

\section{To find the contents of a Cask by the Mean Diameter.}

Rule. - Multiply the difference of the head and bung diameters by .68 for the first variety; by .62 for the second; by .55 for the third; and by .5 for the fourth, when the difference between the head and bung diameter is less than 6 inches; but when the difference between these exceeds 6 inches, multiply that difference by .7 for the first variety; by .64 for the second; by .57 for the third; and by .52 for the fourth. Add this product to the head diameter, and the sum will be a mean diameter. Square this mean diameter, and multiply the square by the length of the cask; this product multiplied or divided by the proper multiplier or divisor, will give the contents.

1. What are the contents of a spheroidal cask, whose 
length is 40 inches, bung diameter 32 inches, and head diameter 24 inches? Ans. 97.6 gallons.

Solution. $-32-24=8 ; 8 \times 7=5.6 ; 5.6+24=29.6$ $=$ mean diameter $; 29.6 \times 29.6=876.16=$ square $; 876.16$ $\times 40=35046.40$, which being divided by 359.5 , the divisor for imperial gallons, will be equal to 97.6 gallons.

By the gauging rule -

Set 40 on C. to the G. R. 18.79 on D. against 24 on D. stands 64.99 on $\mathrm{C}$. 32 on D. stands 116.2 on $\mathrm{C}$. $+116.2$

3)297.39

99.13 gallons.

Dr. Hutton's General Rule for finding the Contents of Casks.

Add into one sum 39 times the square of the bung diameter, 25 times the square of the head diameter, and 26 times the product of the two diameters; then multiply the sum by the length, and the product again by $.00031 \frac{4}{9}$ for the contents in gallons.

\section{EXAMPLE.}

1. What are the contents of a cask whose length is 40 inches, and the bung and head diameters 32 and 24 ?

$32 \times 32=1024 ; 1024 \times 39=39936$

Ans. 93.4579 gallons.

$24 \times 24=576 ; 576 \times 25=14400$

$32 \times 24=768 ; \quad 768 \times 26=19968$

$74304 \times 40=2972160$

$.00031 \frac{4}{9}$

93.4579

Ullaging is the art of finding what quantity of liquor is contained in a cask when partly empty. And it is consid- 
ered in two positions ; first, as standing on its end; secondly, lying on its side.

\section{To find the Contents of Ullage by the Sliding Rule.}

By one of the preceding problems find the whole contents of the cask. Then set the length on N. to 100 on S. $\mathrm{S}$. for a segment standing, or set the bung diameter on $\mathrm{N}$. to 100 on S. L. for a segment lying; then against the wet inches on N. is a number on S. S. or S. L. to be reserved. Next set 100 on B. to the reserved number on A.; then against the whole contents on $\mathrm{B}$. will be found the ullage on $\mathrm{A}$.

\section{QUESTIONS FOR EXERCISE.}

1. What are the contents of 20 pieces of timber 8 inches $\times 12$ inches, and 36 feet long in cubic feet, and also in superficial feet?

2. What number of cubic feet in a log whose quarter girt is $17 \frac{1}{2}$ inches and length 18 feet?

3. What are the contents of $24 \operatorname{logs} 16$ feet long whose quarter girt is 27 inches?

4. Required the tonnage of a ship by the English and American rules, the length of the keel being 125 feet and the breadth of the beam 42 feet?

5. What is the weight of a piece of hackmatack timber 8 inches $\times 10$ inches and 28 feet in length?

6. Required the number of tons in 16 pieces of timber 24 feet long and 12 inches $\times 16$ inches?

7 . In 2,500 feet running length of 2 inches $\times 10$ inches, how many feet of board measure?

8. In 300 feet running length of 10 inch $\times 12$ inch timber, how many tons?

9. What are the contents of a cask of the first variety in wine and ale gallons, whose length is 50 inches, bung diameter 38 inches, and head diameter 30 inches?

10. If a $\log$ be 35 inches in diameter, what is the largest piece of square timber that can be sawed from it? 
11. What difference is there between a floor 28 feet long $\times 20$ feet broad, and two others, each of half the dimensions; and what do the three floors come to @ $\$ 9.00$ per 100 square feet?

Ans. $\$ 75.60$.

12. An elm plank is 14 feet 3 inches long, and it is desired that just a square yard may be slit off from it; at what distance from the edge must the line be struck?

Ans. $7 \frac{99}{171}$ inches.

13. A joist is 7 inches wide and $2 \frac{1}{2}$ inches thick, but a scantling just as big again, that shall be 3 inches thick, is wanted; what will the other dimension be?

Ans. $11 \frac{2}{3}$ inches.

14. The perambulator is so contrived as to turn just twice in $16 \frac{1}{2}$ feet ; required the diameter? Ans. 2.626 feet.

15. In turning a chaise within a ring of a certain diameter, it was observed that the outer wheel made two revolutions while the inner made but one ; the wheels were both 4 feet high, and supposing them fixed at the distance of 5 feet asunder on the axletree, what was the circumference of the track described by the outside wheel? Ans. 63 feet nearly.

16. Having a rectangular board 58 inches by 27 inches, I would have a square foot cut off parallel to the shorter edge; I would then have the same quantity cut from the remainder, parallel to the longer, and this alternately repeated, till there shall not be the quantity of a foot left; what will be the dimensions of the remaining piece?

Ans. 20.7 inches by 6.086 .

17. What is the length of a chord which cuts off $\frac{1}{3}$ of the area of a circle, whose diameter is 289 ?

Ans. 278.6716.

18. What will the diameter of a globe be, when the solidity and superficial contents are expressed by the same number?

Ans. 6.

19. A gentleman has a garden 100 feet long and 80 feet broad, and a gravel walk is to be made of an equal width half round it; what must be the breadth of the walk to take up just half the ground?

Ans 25.968 feet. 
20. How many 3 -inch cubes may be cut out of a 12-inch cube?

Ans. 64.

21. How high above the earth must a person be raised that he may see one third of its surface?

Ans. To the height of the earth's diameter.

22. How many feet of boards would cover the surface of the earth, its diameter being 7,958 miles; and how many solid feet in it?

Ans. $\left\{\begin{array}{c}5,546,407,680,000,000 . \text { No. of } \\ \text { feet of boards to cover it. } \\ 37,416,291,092,323,844,085,000 . \\ \text { No. of cubic feet in the earth. }\end{array}\right.$

23. If the diameter of a circle be 50 feet, what is the circumference of it?

24. Two pillars standing on a horizontal plane are 120 feet asunder; the height of the higher is 100 feet, and that of the lower 80 ; whereabout in the plane must a person place himself, so that his distance from the top of either of the pillars shall be equal to the distance between them?

Ans. 91.78 feet from the bottom of the lower. 69.92 feet from the bottom of the other.

25. Three ships are equally distant from an island, the first ship is 30 miles from the second, the second is 25 miles from the third, and the third is 20 miles from the first ; required the distance to the isle?

Ans. 15.118579 miles from each.

26. Prove that the elevation of the North or Polar star above the horizon is equal to the latitude of the place where its altitude is taken.

27. I have a board in the form of a triangle; the length of one of its sides is 16 feet. I wish to sell one half of it; at what distance from the larger end must it be divided parallel to the larger end.

Ans. 4.68 feet.

28. In 2,500 feet running lengths of 7 inches $\times 9$ inches, how many feet running lengths of $2 \frac{1}{2}$ inches $\times 11$ ? 


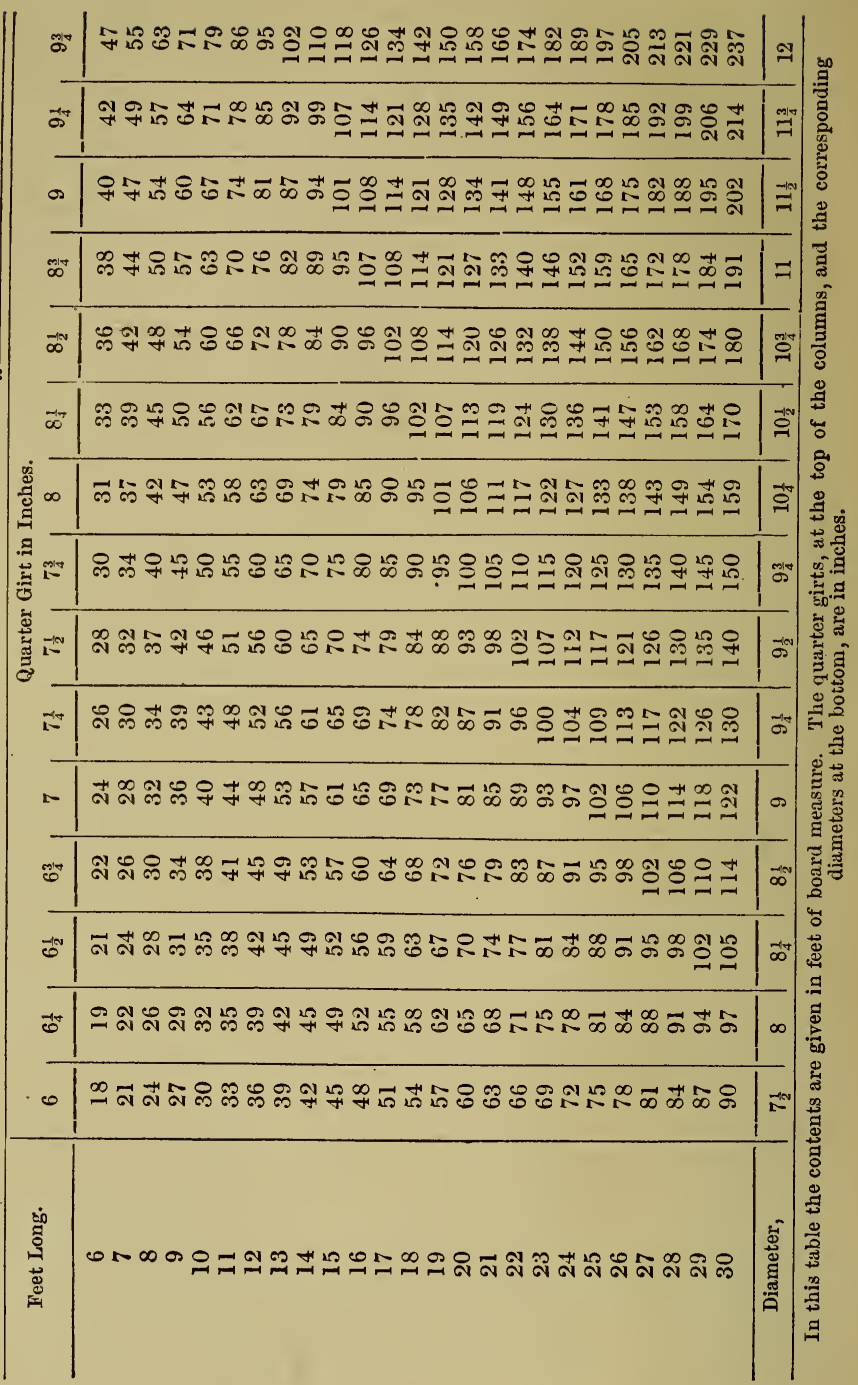




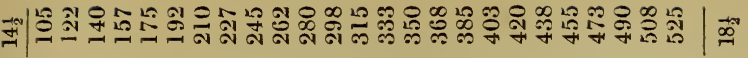

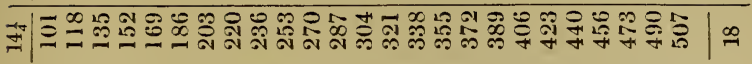

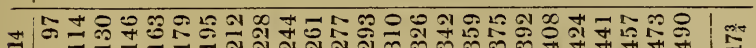

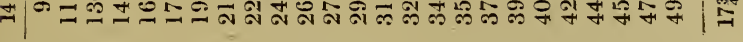

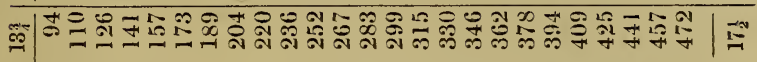

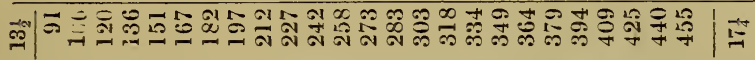

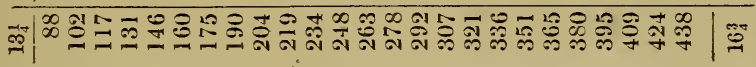

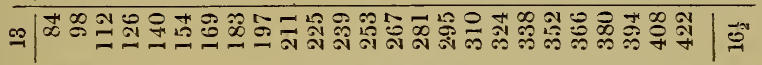

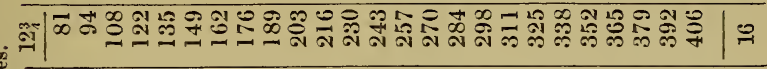

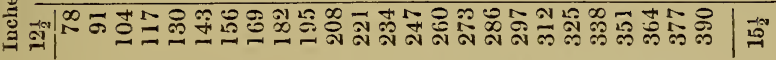

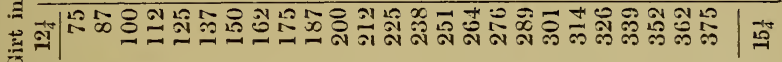

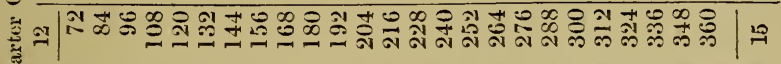
ב्ב

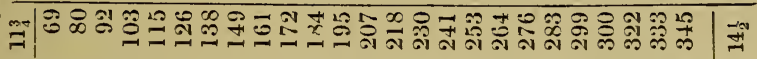

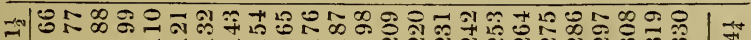
\#|

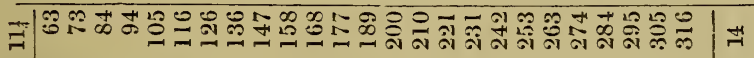

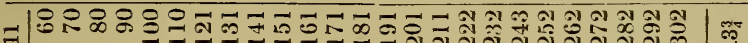
प्र

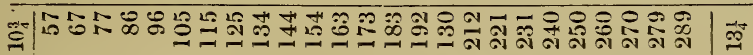

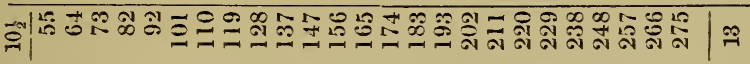

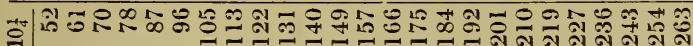
ำ

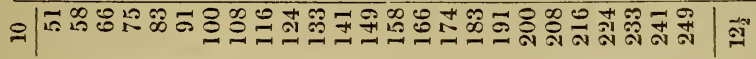

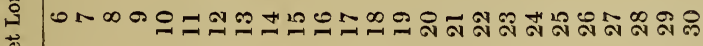
ॐ 
ส|

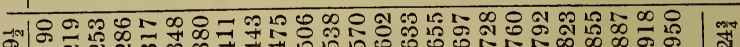

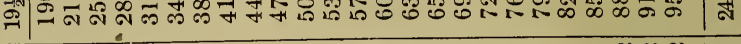

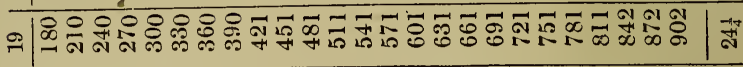

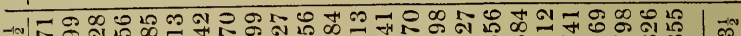

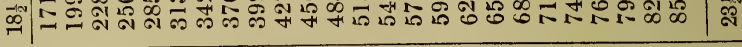

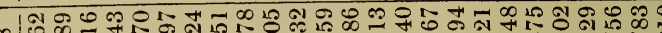

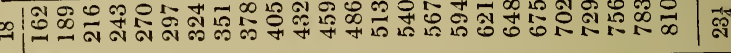

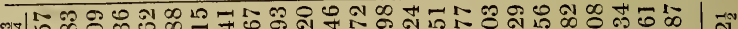

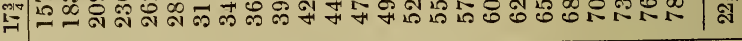
少|

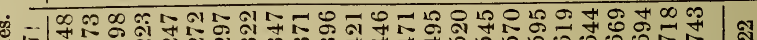

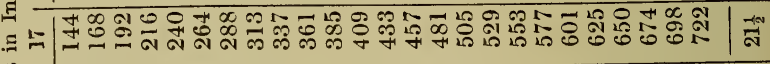

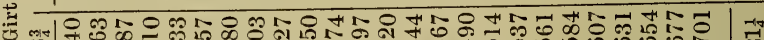

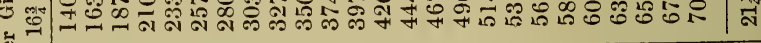

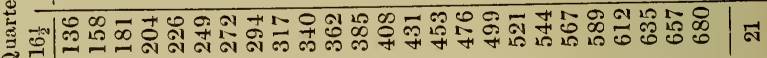

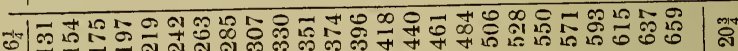

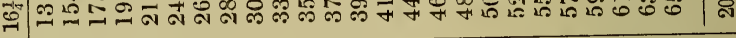

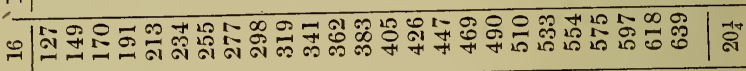

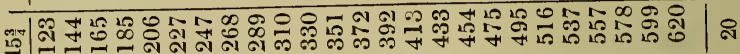
1

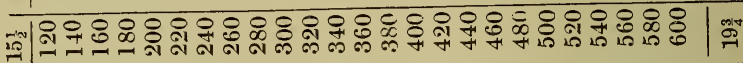

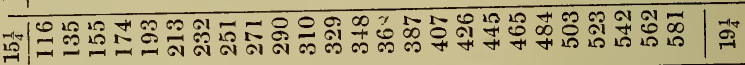

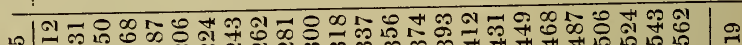

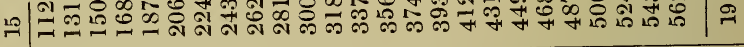

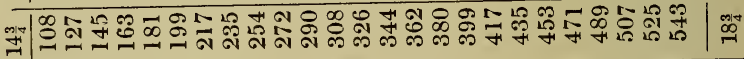
हैं 7 :

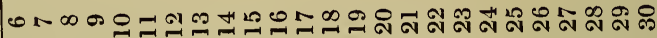




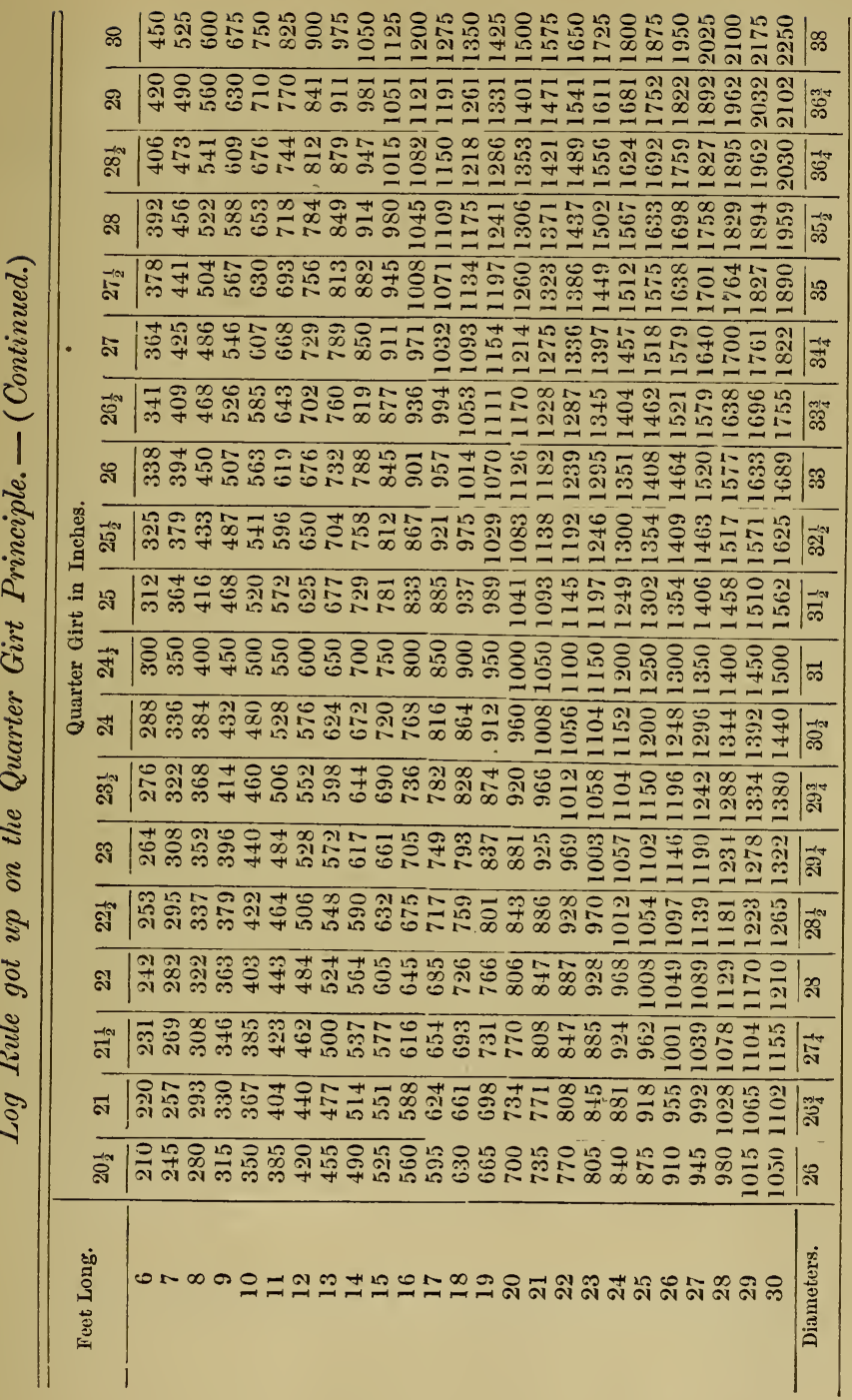




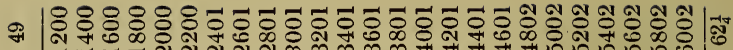

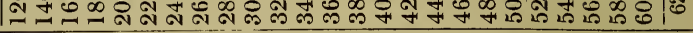

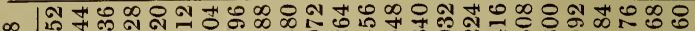

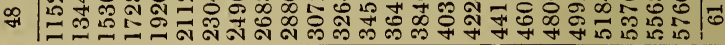

सक

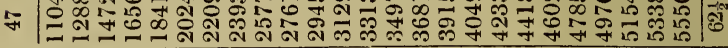

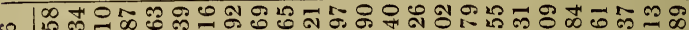

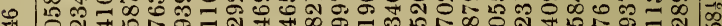

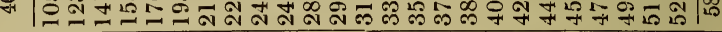

రి

ᄂ

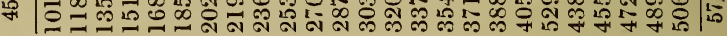

ఘ 年 ঋ

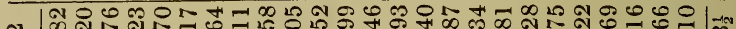
ม $\mid \begin{aligned} & \infty \\ & \infty\end{aligned}$ 急 잉 .

: 尊

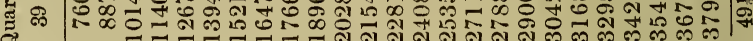
8

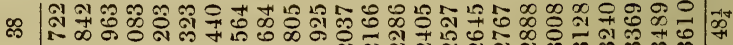

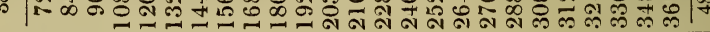
ธ 政

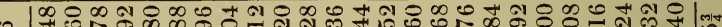

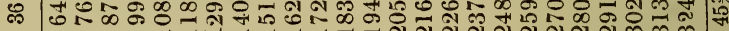

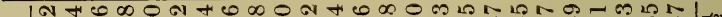

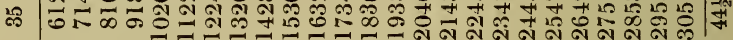

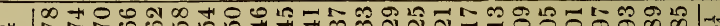
ल

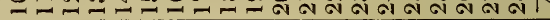

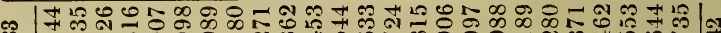
๓

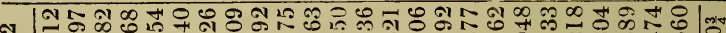

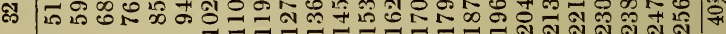
" $\mid$ | 


\section{How to use the Log or Timber Rule.}

If the timber is tapering, the girt should be taken about one third the distance from the larger to the smaller end. Some take the girt in the middle. Girt the log to be measured, and take the quarter of it, and measure the length of the log. Then look along the top of the table till you come to the corresponding quarter girt; then run down the column underneath the quarter girt till you get opposite the length, where you will find the contents. Or, you can find the contents by taking the diameter of the small end and the length. Then find the corresponding diameter at the foot of the table, and ascend the line perpendicularly till you come opposite the length, where you will find the contents.

P. S. - This table allows one fourth of the true contents of the log for bark, saw kerf, and waste slab. It has been extensively used by timber merchants, and is just about as fair a rule to go by as any I have seen. There are many allowances to be made which are left to the scaler's judgment, and for which it would be almost impossible to make due allowance in the table.

\section{INTEREST.}

Rule for finding the interest at 6 per cent. - Multiply the sum by the number of days, divide the product by 6 , then strike off the right-hand figure.

. EXAMPLE.

$\$ 200$

12 days.

6) 2400

$400=40$ cents is the interest. 


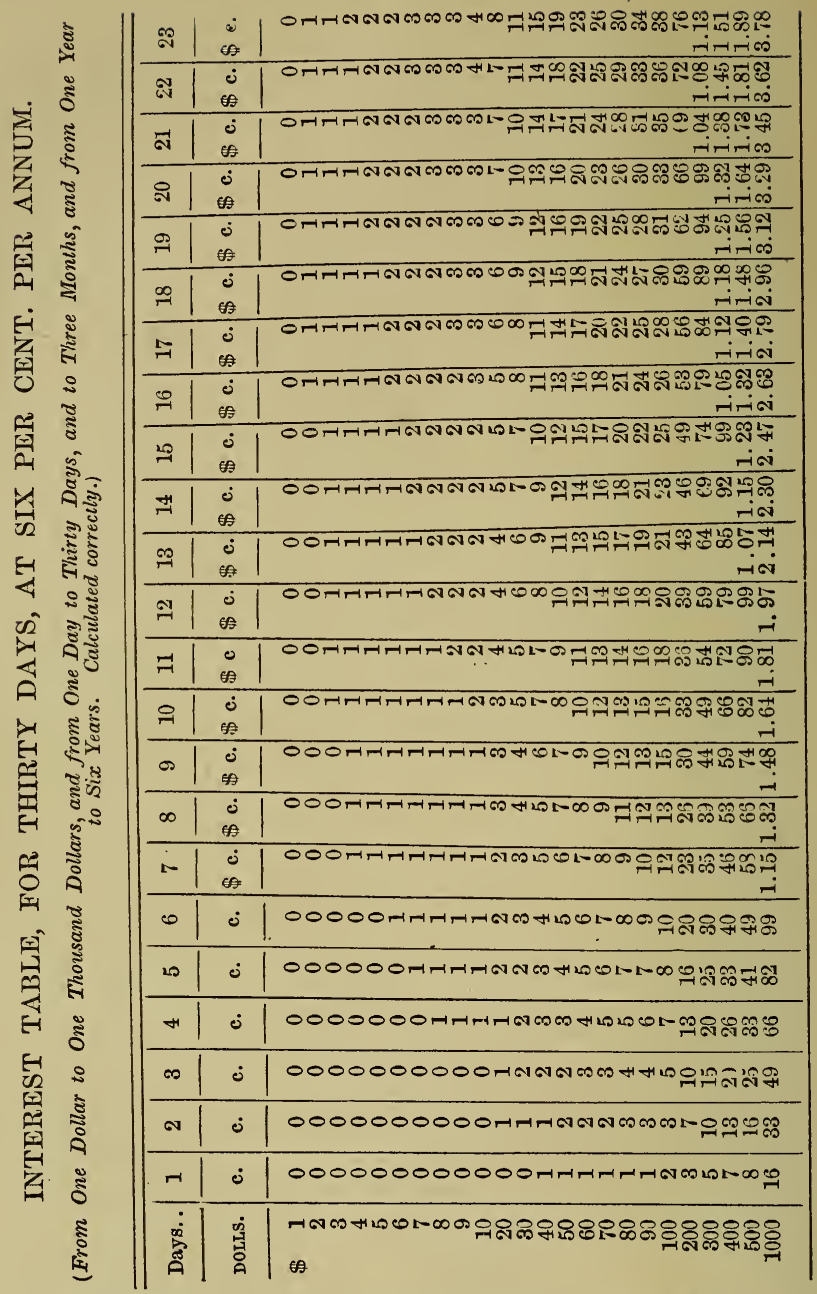

First Example at 6 per cent. - Required, 50 days interest on $\$ 100$. Interest on $\$ 100$ for 30 days $=49$ cents. Interest on $\$ 100$ for 20 days $=33$ "

Ans. $\overline{82}$ cents. 
INTEREST.

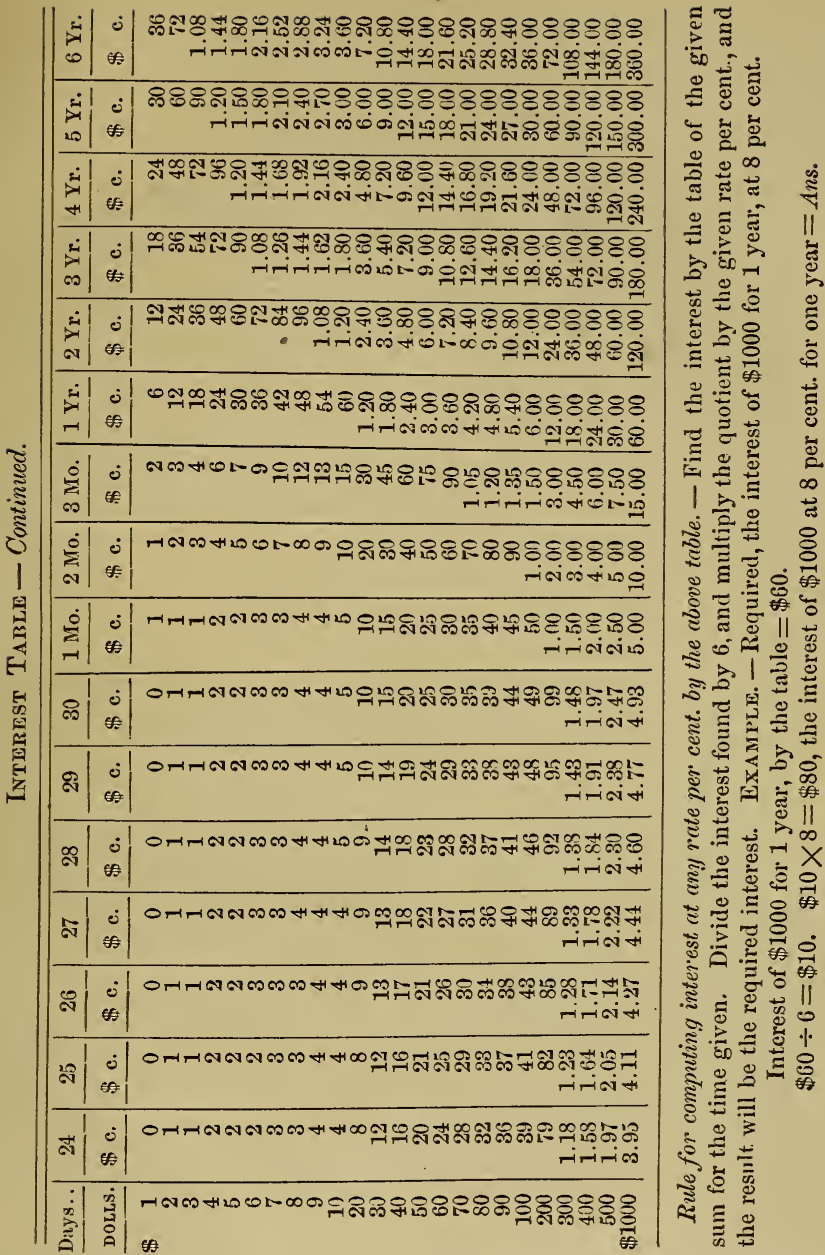

Second Example at 6 per cent. - Required, the interest of $\$ 50$ for 3 years, 2 months, and 10 dars.

Interest on $\$ 50$ for 3 years $=\$ 9.00$

Interest on $\$ 50$ for 2 mos. $=\quad 50$

Interest on $\$ 50$ for 10 days $=8$

Ans. $\overline{\$ 9.58}$ 


\section{WANTED.}

AGents to sell this Book, throughout the United States, the Dominion of Canada, California, and Oregon. Exclusive territory given. Good inducements to agents. The book will be sent to any address, free of postage, on receipt of Two Dollars. Send Post-Office orders, or by express. Address

\section{CHARLES KINSLEY,}

Calais, Me., or St. Stephen, N. B.

\section{NOTE.}

Acl Lumber Manufacturers, Lumber Dealers, Millmen, Carpenters, Carriage Makers, Shipbuilders, Cabinet Makers, Ship Brokers, Ship Carpenters, Railroad Conductors, Engineers, Machinists, Freight Agents, Teachers, Students, Architects, Merchants, Accountants, and others, will find it to their advantage to procure a copy of this book, as the knowledge it imparts may save them in a few years' practice hundreds of dollars. The book contains twelve new rules for finding the superficial contents of lumber, which do the same work as one hundred and fifty of the rules generally used.

CHARLES KINSLEY. 






\section{LIBRARY OF CONGRESS}

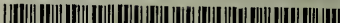

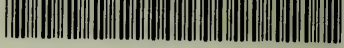

00008982545 